\title{
Electrical Impedance Tomography for Perfusion Imaging and Monitoring
}

\author{
by \\ Symon George Allan Stowe
}

A thesis submitted in partial fulfillment of the requirements for the

degree of

Ph.D.

in

Biomedical Engineering

\author{
Carleton University \\ Ottawa, Ontario
}

(C) 2021

Symon Stowe 
To my family. 


\begin{abstract}
Electrical impedance tomography (EIT) is a medical imaging technology that uses boundary electrodes to inject stimulus currents and measure the resulting potential distributions. These potentials are measured using electrodes which are in turn used to reconstruct conductivity changes within the body. EIT has been studied for its ability to image both the flow of blood and the delivery of blood to a tissue (perfusion). However, cardiac-related signals are challenging to image accurately due to their small amplitude and the limited sensitivity of EIT systems to impedance changes deep in the body. This thesis presents techniques to improve perfusion imaging with EIT by generating more accurate meshes and using internal electrodes to obtain higher sensitivity in the centre of the body. This work develops a tool to generate accurate, customized meshes from diagnostic computed tomography (CT) images, and a technique to reconstruct images with internal electrodes. Custom models reconstructed the location of impedance changes due to ventilation with higher accuracy, and internal electrodes yielded an increase in internal sensitivity over traditional external configurations. Shifting the position of electrodes on an internal probe by as little as $1 \%$ of the tank radius in simulation created artefacts in images reconstructed using existing approaches without motion correction. A novel technique to correct for probe motion is presented that improved reconstruction accuracy and reduced background noise compared to existing techniques. The presented work contributes to increasing internal sensitivity of EIT measurements and demonstrates that refined meshes and internal electrodes may improve measures of perfusion and help to make EIT a viable tool for continuous perfusion monitoring at the bedside.
\end{abstract}




\section{Acknowledgements}

Thank you to the many people who have supported me throughout the last 4 years.

I would like to thank my supervisor Professor Andy Adler for his guidance and support throughout my time at Carleton, and for presenting me the opportunity to be involved in so many interesting projects.

Thank you to Dr. Étienne Fortin-Pellerin and the team at Université de Sherbrooke for their support with so many experiments, and welcoming me into their lab.

I would also like to thank Dr. Alistair Boyle for his valuable insights into the field of EIT and my colleagues who have made my time at Carleton so enjoyable.

Finally, a huge thank you to my family. My parents, Marlys and Doug, and my sisters, Alyssa and Caitlyn have supported this work in so many ways, both in the workshop helping with prototypes and proofreading. A special thank you to my partner Sana for her patience, help and support along this journey. 


\section{Contents}

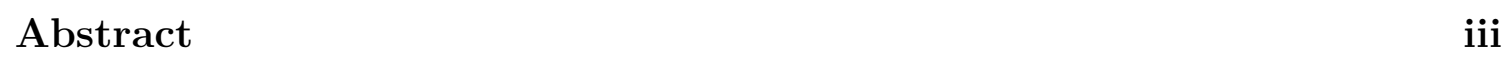

Acknowledgements iv

Contents $x$

List of Figures $x$

List of Tables $\quad$ xiii

list of Acronyms xiv

$\begin{array}{lll}1 & \text { Introduction } & 1\end{array}$

1.1 Problem . . . . . . . . . . . . . . . . . . . . . . . . . . 2

1.2 Thesis Objectives . . . . . . . . . . . . . . . . . . . . . . 4

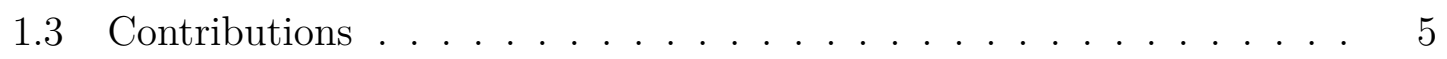

1.3 .1 Publications . . . . . . . . . . . . . . . . . 7

1.3.1.1 Published Works Related to the Thesis . . . . . . 7 
\begin{tabular}{|lrl}
\hline 2 & Background & 8
\end{tabular}

2.1 Impedance Imaging $\ldots \ldots \ldots \ldots$

2.2 Bioimpedance . . . . . . . . . . . . . . . . . . . . . . . 9 9

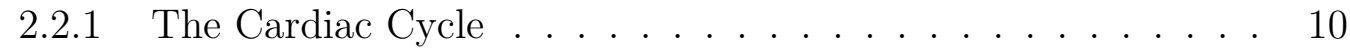

$2.2 .2 \quad$ Bioimpedance of Perfusion . . . . . . . . . . . . . . . . . . . . 14

2.3 Perfusion Imaging . . . . . . . . . . . . . . . . . . . . . . . . . . . . . 15

$2.3 .1 \quad$ Nuclear Medical Imagaing . . . . . . . . . . . . . . . . . . 16

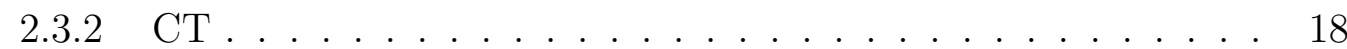

$2.3 .3 \quad \mathrm{MRI} \ldots \ldots \ldots \ldots \ldots$

$2.3 .4 \quad \mathrm{EIT} \ldots \ldots \ldots \ldots \ldots$

2.4 Electrical Impedance Tomography . . . . . . . . . . . . . . . . . . . . 23

$2.4 .1 \quad$ EIT Measurements . . . . . . . . . . . . . . . . . . . . . 23

2.4.1.1 Electrodes . . . . . . . . . . . . . . . . 23

2.4.1.2 Current Injection and Voltage Measurement. . . . . 24

2.4 .2 Imaging Techniques . . . . . . . . . . . . . . . 26

2.4 .3 Forward Problem . . . . . . . . . . . . . . . . . . . . . . . . . 27

2.4 .4 Discretization and the Finite Element Method . . . . . . . . . 30

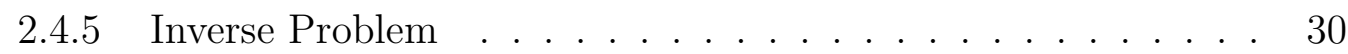

2.4.5.1 Regularization . . . . . . . . . . . . . 31

2.4.5.2 Image Reconstruction . . . . . . . . . . . . . . 32

2.4.5.3 Image Reconstruction With GREIT . . . . . . . . . 33

$2.4 .6 \quad$ Internal Electrodes . . . . . . . . . . . . . . . . . . . . . . . . 35

2.5 Summary $\ldots \ldots \ldots \ldots$. . . . . . . . . . . . . . . . . . . . 37 
3 Bolus- and Frequency-Based Perfusion 38

3.1 Introduction . . . . . . . . . . . . . . . . . . . . . . . 38

3.2 Methods . . . . . . . . . . . . . . . . . . . . . . . . . . . 43

3.2.1 $\quad$ Overview . . . . . . . . . . . . . . . . . . . . . 43

3.2 .2 Animals . . . . . . . . . . . . . . . . . . . 43

3.2.3 Data Acquisition and Image Reconstruction . . . . . . . . . . 46

3.2 .4 Functional EIT Images . . . . . . . . . . . . . . . . . . . . 47

3.2.4.1 Bolus Injection Image $\left(\mathrm{P}_{\mathrm{B}}\right) \ldots$. . . . . . . . . 47

3.2.4.2 $\quad$ Frequency-Filtering . . . . . . . . . . . . . . . 48

3.2.4.3 Ensemble Averaging . . . . . . . . . . . . . . . . 50

3.2.5 Image Comparison . . . . . . . . . . . . . . . . . 51

3.2 .6 Statistical Analysis . . . . . . . . . . . . . . . . . . 52

3.3 Results . . . . . . . . . . . . . . . . . . . . . 52

3.4 Discussion . . . . . . . . . . . . . . . . . . . . . 56

3.5 Summary . . . . . . . . . . . . . . . . . . 60

$4 \quad$ FEM Mesh Refinement for 3D EIT 62

4.1 Motivation . . . . . . . . . . . . . . . . . . . . . . . 62

4.2 Introduction $\ldots \ldots \ldots \ldots \ldots$. . . . . . . . . . . . . . . . . . . . . . 62

4.3 Methods . . . . . . . . . . . . . . . . . . . . . 65

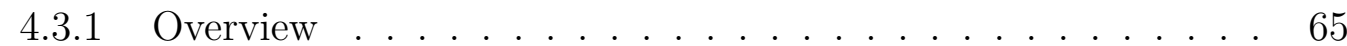

4.3 .2 Mesh Generation . . . . . . . . . . . . . . . . . 65

4.3 .3 Simulation . . . . . . . . . . . . . . . . . 71

4.3.4 Electrode Refinement for Arbitrary FEMs . . . . . . . . . . . 71 
4.4 Results . . . . . . . . . . . . . . . . . . . . . . . . . . . . . . . . . . . . .

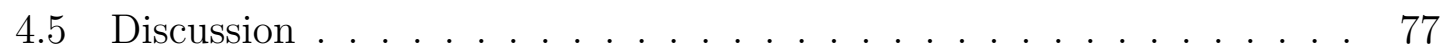

4.6 Summary $\ldots \ldots \ldots \ldots \ldots \ldots$

5 Custom EIT Meshes $\quad 82$

5.1 Introduction . . . . . . . . . . . . . . . . . . . . . . 82

5.2 Methods . . . . . . . . . . . . . . . . . 86

$5.2 .1 \quad$ Automatic Segmentation of the Thorax . . . . . . . . . 88

$5.2 .1 .1 \quad$ External Boundary . . . . . . . . . . . . . . . 89

$5.2 .1 .2 \quad$ Chest Cavity . . . . . . . . . . . . . . . 90

5.2 .1 .3 Lungs $\ldots \ldots \ldots \ldots \ldots$

$5.2 .2 \quad$ Manual Segmentation Correction . . . . . . . . . . . . . . 95

5.2 .3 Mesh Generation . . . . . . . . . . . . . . . . . . . . . 98

$5.2 .4 \quad$ Evaluation on ARDS Patients . . . . . . . . . . . . . . . 99

5.3 Results . . . . . . . . . . . . . . . . . . . . . . . 100

5.4 Discussion $\ldots \ldots \ldots \ldots \ldots \ldots$

5.5 Summary $\ldots \ldots \ldots \ldots \ldots \ldots$

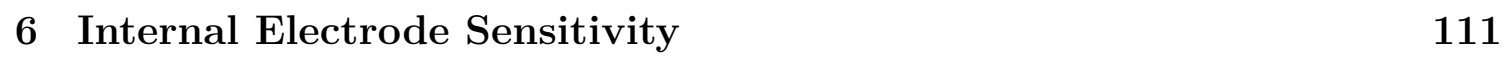

$6.1 \quad$ Introduction . . . . . . . . . . . . . . . . . . . . . . . . 111

6.2 Methods . . . . . . . . . . . . . . . . . . . . . . . . . . . 114

6.2 .1 Tank Model . . . . . . . . . . . . . . . . . . . . . . . . . . . . 114

6.2 .2 Image Reconstruction . . . . . . . . . . . . . . . . . 115

6.2 .3 Sensitivity Calculation $\ldots \ldots \ldots \ldots$

viii 
$6.2 .4 \quad$ Current Injection and Measurement. . . . . . . . . . . . 116

6.3 Results. . . . . . . . . . . . . . . . . . . . . . . . . . 117

6.3 .1 Sensitivity in an Ovine Model . . . . . . . . . . . . . . . . . . 121

6.4 Discussion . . . . . . . . . . . . . . . . . . . . . . . . . . . . . . . . . . 121

6.5 Summary $\ldots \ldots \ldots \ldots \ldots \ldots \ldots \ldots$

$\begin{array}{lll}7 & \text { Internal Electrode Motion } & 124\end{array}$

7.1 Introduction . . . . . . . . . . . . . . . . . . . . . . . . 124

7.2 Methods . . . . . . . . . . . . . . . . . . . . . . . . . 127

$7.2 .1 \quad$ Simulations . . . . . . . . . . . . . . . . . . . . 128

7.2.1.1 Tank Model . . . . . . . . . . . . . . . . . . 128

7.2 .1 .2 Measurements . . . . . . . . . . . . . 130

$7.2 .2 \quad$ Movement Correction . . . . . . . . . . . . . . . . 131

7.2 .3 Image Comparison $\ldots \ldots \ldots \ldots \ldots$

$7.2 .4 \quad$ In-vivo Recordings $\ldots \ldots \ldots \ldots \ldots$

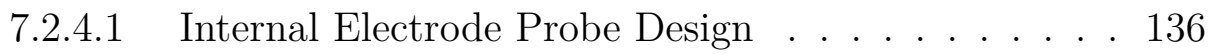

7.2.4.2 Electrode Placement . . . . . . . . . . . . . . . . 138

7.2 .4 .3 Ovine Mesh . . . . . . . . . . . . . . 138

7.2.4.4 Reconstruction . . . . . . . . . . . . . . 140

7.2.4.5 Pulsatile Amplitude . . . . . . . . . . . . . . 141

7.3 Results . . . . . . . . . . . . . . . . . . . . . . . . . . . . 141

7.3 .1 Simulation . . . . . . . . . . . . . . . . . . . . 141

7.3 .2 In-vivo . . . . . . . . . . . . . . . . . . 146

7.4 Discussion . . . . . . . . . . . . . . . . . . . . . . . . 148 
7.5 Summary . . . . . . . . . . . . . . . . . . . . . 153

8 Conclusion 154

$8.1 \quad$ Summary of Findings . . . . . . . . . . . . . . . . . . . . 155

8.2 Future Work . . . . . . . . . . . . . . . . . . . . . . . 157

8.3 Conclusion $\ldots \ldots \ldots \ldots \ldots \ldots$

\begin{tabular}{ll}
\hline Bibliography & 161
\end{tabular}

\begin{tabular}{ll}
\hline Appendices & 182
\end{tabular}

\begin{tabular}{ll}
\hline A GMSH models & 183
\end{tabular}

A.1 Tank model . . . . . . . . . . . . . . . . . . . . . . . . . . . . . 183

A.2 Internal probe model . . . . . . . . . . . . . . . . . . . 184

\begin{tabular}{ll}
\hline B Algorithms & 188
\end{tabular}

B.1 External boundary . . . . . . . . . . . . . . . . . . . . . . . . . . 188

B.2 Ribcage . . . . . . . . . . . . . . . . . . . . . . . . . . . . . 189

B.3 Lungs . . . . . . . . . . . . . . . . . . . . . . . . . . . . . . . . . . . . 191 


\section{List of Figures}

1.1 Overview of thesis objectives . . . . . . . . . . . . . . 5

2.1 Current and equipotential lines . . . . . . . . . . . . . . . . . . . . . 9

2.2 Sketch of the anatomical heart . . . . . . . . . . . . . . . . . . . . . 12

2.3 Example ECG waveform . . . . . . . . . . . . . . . . . . . . 13

2.4 Adjacent and "skip 4" stimulation patterns . . . . . . . . . . . . . . . 26

3.1 Overview of the EIT analysis methods . . . . . . . . . . . . . . 45

3.2 Selection of the bolus signal . . . . . . . . . . . . . . . . . . . . 48

3.3 Frequency analysis methods . . . . . . . . . . . . . . . . . 50

3.4 Ensemble averaging analysis . . . . . . . . . . . . . . . . . 51

3.5 Jaccard index scores . . . . . . . . . . . . . . . . 53

3.6 Example perfusion images . . . . . . . . . . . . . . . . . 55

$4.1 \quad$ Example meshes for various refinement strategies . . . . . . . . . . . 67

4.2 Mesh size surrounding the electrode . . . . . . . . . . . . . . . . . . . 68

$4.3 \quad$ Balance point calculation method . . . . . . . . . . . . . . . . . 69

4.4 Mesh sensitivity error vs. elements per electrode . . . . . . . . . . . . 73 
4.5 Sensitivity distribution and regions of interest . . . . . . . . . . . . . 74

4.6 Sensitivity error with shifting node balance . . . . . . . . . . . . . . . 76

$4.7 \quad$ Advanced mesh example of an internal probe . . . . . . . . . . . . . . 79

5.1 Raw CT images at the $4^{\text {th }}$ intersoctal space . . . . . . . . . . . . 85

5.2 Mesh generation method overiew. . . . . . . . . . . . . . . . . . . 87

5.3 Boundary segmentation methods. . . . . . . . . . . . . . . . . . . 90

5.4 Chest cavity segmentation methods. . . . . . . . . . . . . . . . . . . 92

5.5 Lung segmentation methods. . . . . . . . . . . . . . . . . . . . . . . 94

5.6 Manual segmentation data loading . . . . . . . . . . . . . . 96

5.7 Manual segmentation interface with initial input . . . . . . . . . . . . 97

5.8 Manually corrected segmentation . . . . . . . . . . . . . . . . . 98

5.9 Lung segmentation results . . . . . . . . . . . . . . . . . 101

5.10 Custom and generic meshes . . . . . . . . . . . . . . . . . . 102

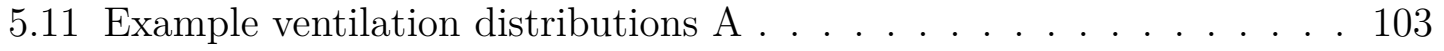

5.12 Example ventilation distributions B . . . . . . . . . . . . . . . 104

5.13 Example ventilation distributions C . . . . . . . . . . . . . . . . 104

5.14 Example ventilation distributions D . . . . . . . . . . . . . . . 105

5.15 Center of mass error . . . . . . . . . . . . . . . . 106

$6.1 \quad$ Internal electrode configurations . . . . . . . . . . . . . . . . . 115

6.2 Current injection patterns with internal electrodes . . . . . . . . . . . 117

6.3 Internal electrode simulation reconstructions . . . . . . . . . . . . . . 118

$6.4 \quad$ Sensitivity with different internal electrode configurations . . . . . . . 119 
6.5 Sensitivity using internal electrodes with modified injection patterns . 120

6.6 Sensitivity distribution in a lamb model . . . . . . . . . . . . . . . . 121

$7.1 \quad$ Spherical and cylindrical internal electrodes $\quad . \quad$. . . . . . . . . . . . 129

7.2 Motion correction methods . . . . . . . . . . . . . . . . . . . . . . . 134

7.3 Probe prototype . . . . . . . . . . . . . . . . . . . 137

$7.4 \quad$ Ovine mesh with organ regions and probe placement . . . . . . . . . 139

7.5 Ovine model with internal probe . . . . . . . . . . . . . . . . . . . . 140

7.6 Results of the probe location correction . . . . . . . . . . . . . . . . . 145

7.7 Reconstrions on 3 ewes . . . . . . . . . . . . . . . . . . . . . . . . 147

$7.8 \quad$ Amplitude of the cardiosynchronous signal . . . . . . . . . . . . . . . 148 


\section{List of Tables}

4.1 Parameters used to generate meshes . . . . . . . . . . . . . . . . 70

7.1 Jaccard scores for reconstructions with electrode probe movement . . 143

7.2 Noise estimates for reconstructions with electrode probe movement - 143 


\section{List of Acronyms}

ARDS Acute Respiratory Distress Syndrome.

bpm Beats per Minute.

CMV Conventional Mechanical Ventilation.

CT Computed Tomography.

ECG Electrocardiogram.

EIT Electrical Impedance Tomography.

FEM Finite Element Model.

FFT Fast Fourrier Transform.

FWHM Full Width Half Max.

GI Global Inhomogeneity.

GUI Graphical User Interface.

ICU Intensive Care Unit.

MRI Magnetic Resonance Imaging.

MSE Mean Squared Error. 
PEEP Positive End-Expiratory Pressure.

PET Positron Emission Tomography.

SNR Signal-to-Noise Ratio.

SPECT Single Photon Emission Computed Tomography.

TLV Total Liquid Ventilation. 


\section{Chapter 1}

\section{Introduction}

Electrical impedance tomography EIT is an imaging modality that uses an arrangement of electrodes to simultaneously apply stimulation currents and measure the resulting electric potentials. Measurements of potential at the electrodes are used in conjunction with prior information to reconstruct images of the internal conductivity. In biomedical applications, the variance in conductivity between different tissue types and fluids enables non-invasive imaging of functional activity using electrodes on the body surface.

Thoracic EIT focuses on imaging cardiopulmonary activity using a belt of electrodes placed around the ribcage. In images of the chest, the signal is often dominated by impedance changes due to air movement in the lungs. The much smaller signals due to blood flow are of great interest for continuous monitoring and diagnostic applications, but are challenging to identify.

This thesis explores techniques to improve sensitivity to blood movement, or per- 
fusion, in EIT through the development of novel meshing techniques and electrode configurations incorporating internal electrodes. We investigate the current limitations of perfusion monitoring in 2D, and analyze novel meshing techniques and electrode placements for their ability to improve thoracic imaging and increase sensitivity to blood flow. The main problems we must overcome to accurately image perfusion with EIT are: the limited sensitivity to cardiosynchronous signals, and challenges identifying the correct region of interest.

\section{$1.1 \quad$ Problem}

EIT can be used to image blood movement in two ways. First, it is possible to track the flow of blood using a conductivity-contrasting agent injected into a vein or artery and second, the signal can be filtered to isolate cardiac-frequency impedance changes. Using a conductive bolus injection to image perfusion has been well established and can give an easily detectable signal. However, contrast agent-based techniques do not allow continuous monitoring, and frequent injections may pose a risk to the patient. In thoracic EIT, bolus injections also typically occur during apnoea to facilitate removal from the respiratory component. Filtering techniques for perfusion imaging are more appealing for monitoring applications as they are less invasive and could be used continuously, but they present several challenges.

The primary challenge of perfusion imaging is the small amplitude of the signal. Impedance changes related to cardiac activity are often an order of magnitude smaller than signals related to respiration, and can be challenging to identify without 
averaging many heartbeats together. When averaging over several heartbeats, the ability of the system to monitor in real time is greatly diminished. Additionally, there is some uncertainty surrounding the source of cardiac-frequency impedance changes. It is unclear to what extent the cardiosynchronous impedance changes stem from pulsatile motion in the thorax, compared to the movement of blood, and if they can be used as a true measure of perfusion.

EIT is minimally invasive, requiring only the application of electrodes on the body surface, but does not have high sensitivity in the central regions of the chest where perfusion changes of interest occur. As electrical current travels through the body, it diffuses away from the electrodes, travelling in three dimensions along the path of least resistance. EIT is most sensitive to impedance changes close to the electrodes, where the current density is highest, and along the path of the injected current.

The placement of electrodes internally could increase current density and therefore sensitivity in the centre of the thorax. The benefits of internal electrodes have been simulated in 2D, showing improvements in reconstruction accuracy and sensitivity (Nasehi Tehrani et al., 2012a), but in practice there are several challenges to overcome. Due to differences in physiology, it is challenging to model the correct location of internal electrodes, and electrodes placed internally may move relative to external electrodes during different physiological processes. To reconstruct images with internal electrodes, an accurate model with precise placement of electrodes is required. The model must also be refined to meet the accuracy requirements of the study. Due to the high sensitivity near the internal electrodes, any errors in this 
region will contribute significant artefacts to the reconstructed image. It is in part due to these challenges that internal electrodes have not been widely used in realworld situations, and to our knowledge, no implementation of internal electrodes in conjunction with a 3D arrangement of external electrodes has been used for in-vivo imaging.

The applications of an EIT system with increased sensitivity to perfusion changes in the thorax are extensive. Methods to monitor blood pressure non-invasively are currently under development and could be improved with increased sensitivity near the region of interest. Increased sensitivity near the heart could enable EIT to be realized as a low-cost solution to monitor and image hemodynamic activity.

\subsection{Thesis Objectives}

The goal of this project is to improve the sensitivity of EIT to perfusion using improved mesh accuracy and custom electrode locations. This thesis approaches the problem from three angles (figure 1.1). We investigate the limitations of current perfusion monitoring techniques, explore advanced meshing techniques, and present novel electrode configurations with internal electrodes and their applications to improve thoracic EIT. 


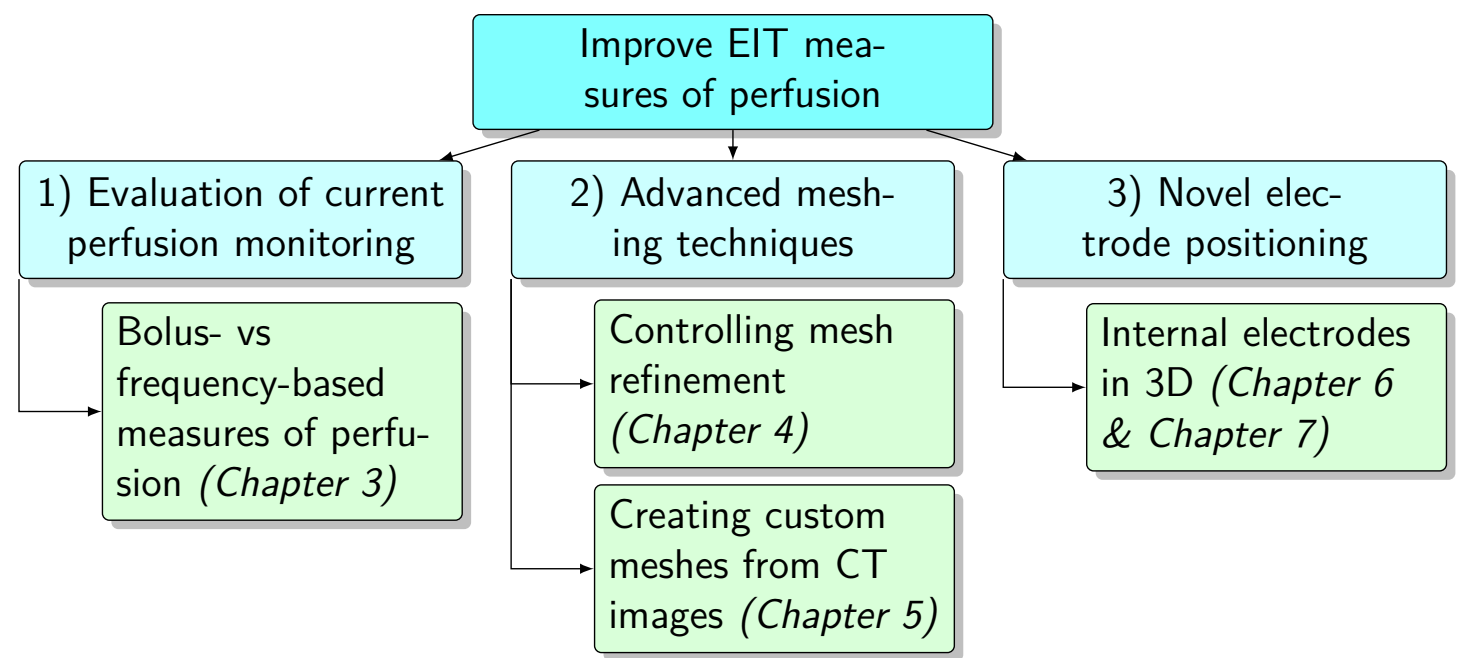

Figure 1.1: This flowchart presents an overview of the thesis objectives. This thesis aims to improve EIT measures of perfusion through 3 avenues. 1) Comparing and investigating the limitations of existing perfusion imaging methods; 2) Advanced meshing techniques; and 3) Using novel electrode positions consisting of a 3D external configuration with internal electrodes.

\subsection{Contributions}

This thesis presents several contributions to the field of perfusion imaging and meshing in EIT.

- A method to determine the node distribution in a finite element model mesh, that reduces error when calculating sensitivity. Chapter 4 presents an analysis between meshing software commonly used in EIT and recommends a technique to place a fixed number of nodes to reduce error when calculating sensitivity. A finer node density has often been suggested near the electrodes due to the higher sensitivity, but no analysis was available to determine how much mesh refinement was required. Using a balance point technique this chapter determines the best distribution of nodes to calculate the sensitivity of voltage 
measurements due to conductivity changes.

- A tool to generate accurate, custom meshes of arbitrary boundaries for EIT reconstruction from $\mathrm{CT}$ images. Chapter 5 documents the design and validation of a tool to generate meshes for EIT image reconstruction from CT images. Diagnostic CT images were automatically segmented, and a tool to manually check the segmentations and generate meshes was created.

- An analysis of 3D electrode placements with internal electrodes on internal sensitivity. Chapter 6 presents an analysis of the sensitivity patterns corresponding to internal electrodes when used with 2 planes of external electrodes. Internal electrodes are expected to increase sensitivity in the centre of the model, but it is not clear to what extent image reconstructions may be improved, or how many electrodes should be placed internally. This chapter provides an analysis of the sensitivity when using internal electrodes in 3D with different current injection and measurement patterns.

- A method to reconstruct images using internal electrode measurements in the presence of motion. Chapter 7 demonstrates the effect of internal electrode probe motion on image reconstruction, and presents a technique using the interference in reconstructed images due to the movement artefact to create a model with a corrected probe location. This technique improves reconstruction performance and reduces noise levels in the presence of electrode motion. Internal electrodes were used successfully to reconstruct images of ventilation in-vivo. This technique could enable accurate EIT image reconstruction using internal electrodes clinically. 


\subsubsection{Publications}

\subsubsection{Published Works Related to the Thesis}

- Stowe, S., Boyle, A., Sage, M., See, W., Praud, J.-P., Fortin-Pellerin, É., \& Adler, A. (2019). Comparison of bolus- and filtering-based EIT measures of lung perfusion in an animal model [Publisher: IOP Publishing]. Physiological Measurement, 40(5), 054002

- Stowe, S., Zhao, Z., Huxter, E., He, H., Long, Y., \& Adler, A. (2021b). Generating forward models from CT images for ventilation monitoring in ARDS patients. Proceedings of the 21st International Conference on Biomedical Applications of Electrical Impedance Tomography, 20

- Stowe, S., \& Adler, A. (2020). The effect of internal electrodes on electrical impedance tomography sensitivity [ISSN: 2694-0604]. 2020 42nd Annual International Conference of the IEEE Engineering in Medicine Biology Society (EMBC), 1457-1460

- Stowe, S., Samson, N., Nadeau, C., Morin, C., Praud, J.-P., Fortin-Pellerin, É., \& Adler, A. (2021a). Using esophageal electrodes for increased sensitivity to cardiac-frequency impedance changes. Proceedings of the 21st International Conference on Biomedical Applications of Electrical Impedance Tomography, 24 


\section{Chapter 2}

\section{Background}

This section presents background on the origin of perfusion signals, reviews current techniques for lung perfusion and hemodynamic monitoring, and provides an overview of EIT as used for thoracic imaging and monitoring.

\section{$2.1 \quad$ Impedance Imaging}

Impedance imaging has been in use since the early 1900s for geophysical applications. Originally introduced as a technique to image below the earth's surface, current was transmitted between two electrodes placed into the ground and any anomalies in subsurface conductivity produced deviation in the equipotential lines. By using multiple locations for current injections and measurements, and the known electrical properties of geological structures, Conrad Schlumberger identified features of underground geological structures (Allaud and Martin, 1977).

These same techniques can be applied in biomedical applications where voltage 
is measured on an array of body surface electrodes while current is applied between select electrode pairs (figure 2.1). Due to impedance differences associated with biological tissues and their physiological function (Geddes and Baker, 1967; McAdams and Jossinet, 1995), EIT has been proposed for a wide range of applications from thoracic monitoring (Frerichs et al., 2017) to neuronal and brain imaging (Holder, 1992).

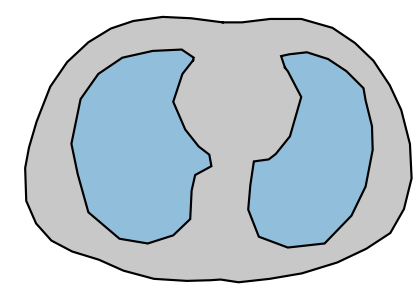

(A)

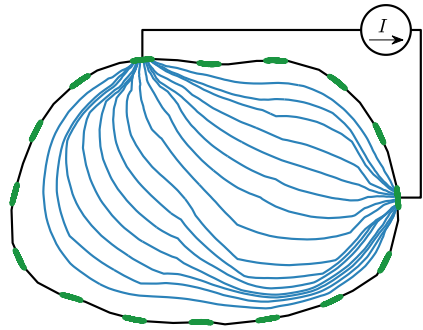

(B)

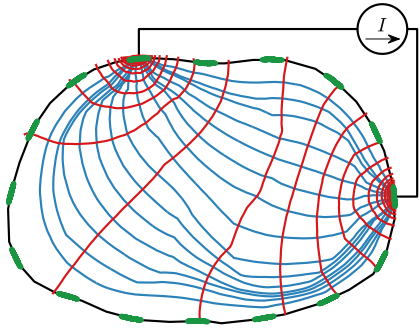

(C)

Figure 2.1: (A) A body comprising tissues of different conductivity, (B) Electrodes are placed on the surface and current is injected between a pair or electrodes. The current pathways are indicated by the blue lines. (C) The resulting equipotential lines within the body are shown in red.

\subsection{Bioimpedance}

In thoracic imaging, the most commonly measured impedance changes occur due to the movement of air in the lungs, the flow of blood and the motion of organs (Adler and Boyle, 2017). During inhalation, the volume of air in the lungs increases, lowering the conductivity of the lung tissue. The resistivity of lung tissue varies significantly between expiration and inspiration, giving a value of $7 \Omega \mathrm{m}$ during expiration 
and $23 \Omega \mathrm{m}$ during inspiration at $100 \mathrm{kHz}$ (Witsoe and Kinnen, 1967), resulting in a measurable variation in impedance during respiration (Eyüboğlu et al., 1989). There are also other significant sources of impedance change that make EIT signal interpretation challenging. For example, simulations have attributed up to $20 \%$ of the respiratory signal to the effect of chest expansion and movement of the chest wall (Adler et al., 1994).

The source of impedance changes due to the flow of blood is even more complex. Since the resistivity of blood is so much lower than other tissues $(1.5 \Omega \mathrm{m})$, the increase of blood due to pulsatile flow decreases the impedance of structures it passes through by a detectable amount (Eyüboğlu et al., 1989). It is often assumed that the component of EIT images at the cardiac frequency is related to the perfusion of blood, but the exact source of cardiosynchronus EIT signals is unclear (Nguyen et al., 2012; Patterson, 2010). A continuous flow of blood alone is insufficient to induce a significant impedance change, as the volume and concentration of the conductive medium is unchanged. The movement of structures within the body may contribute significantly to the measured impedance change (Braun et al., 2018b). Any impedance-based measure of perfusion relies on the cardiosynchronous EIT signals, which have numerous possible sources (Adler et al., 2017b).

\subsubsection{The Cardiac Cycle}

The cardiac cycle consists of the activity in the heart between the beginning of one heart beat, and the next. There are two main stages of the cardiac cycle: the diastole, when the heart relaxes and is filled with blood, and the systole, when contraction 
of the heart pumps blood to the lungs and all other body systems (Pappano and Wier, 2019). A simplified anatomy of the heart is presented in figure 2.2. Since ECG recordings are frequently used to synchronize EIT data, it is helpful to look at the timing of the cardiac cycle as it relates to features of ECG traces. An example ECG waveform is pictured in figure 2.3 . 


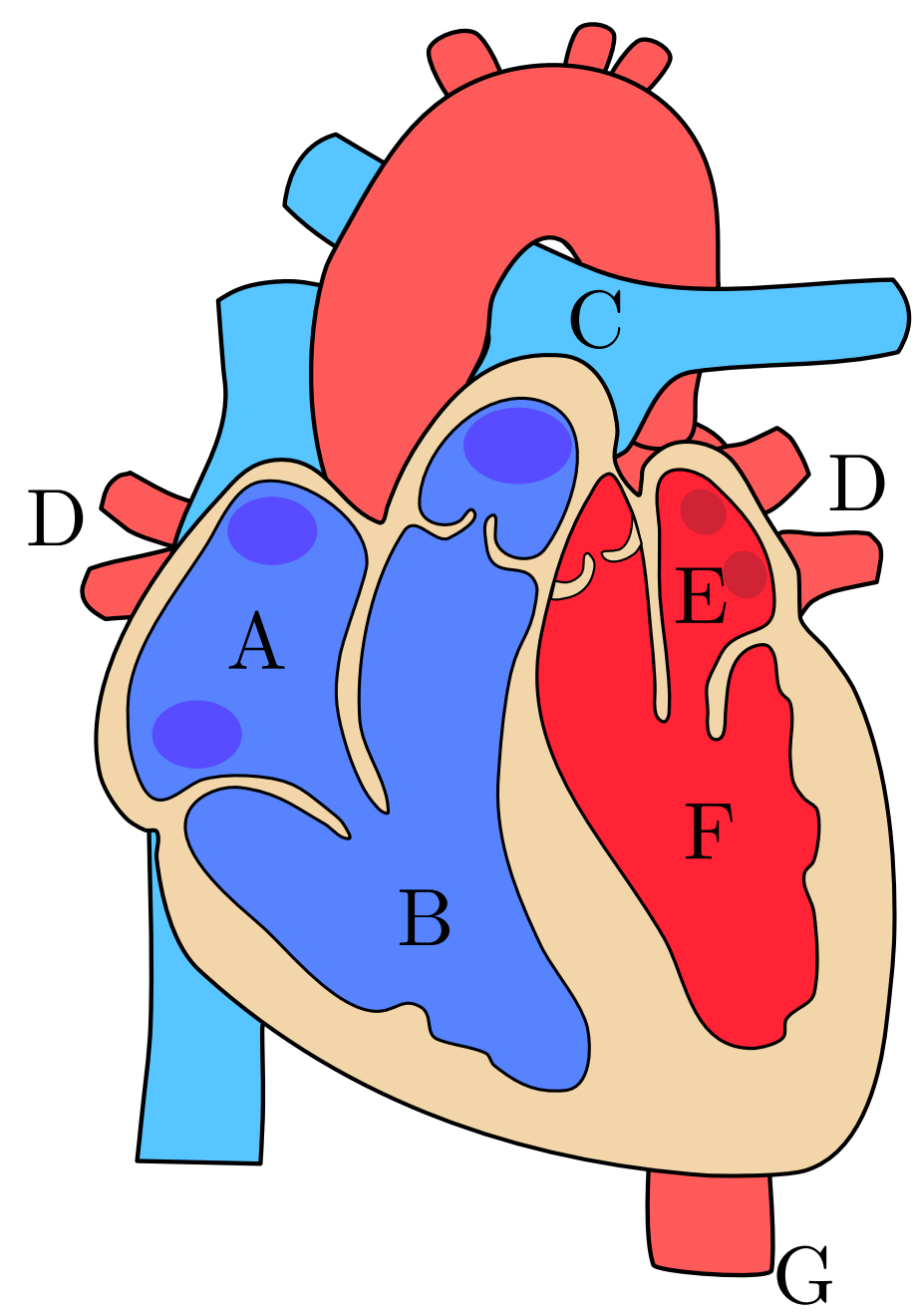

Figure 2.2: A sketch of the heart prepared for this thesis shows the pathway of the blood from entry to the heart, to the descending aorta. Deoxygenated blood enters the heart through the right atrium (A), where it is moved to the right ventricle (B). From there it passes through the pulmonary artery $(\mathrm{C})$ into the lungs where it perfuses and is oxygenated. Blood returns from the lungs through the pulmonary veins (D), then enters the left atrium (E). Finally, the blood is pumped from the left ventricle (F) to the rest of the body through the aorta, and the descending aorta $(\mathrm{G})$.

During the first stage of the cardiac cycle is the ventricular diastole, during which the heart relaxes and expands. Blood is pulled into the ventricles from the atria as the tricuspid and mitral valves open. The $\mathrm{P}$ wave occurs due to atrial depolarization 
during the diastole. Blood enters the atrium on the right side of the heart through the superior and inferior vena cava and on the left side of the heart, the atrium is filled by oxygenated blood from the lungs through the pulmonary veins (Pappano and Wier, 2019). Next is the atrial contraction during which time the atria pump additional blood into the ventricles, and the ventricular volume and pressure are maximized. At the peak of the ventricular volume, the ventricles contract and depolarize which corresponds to the QRS complex (Pollock and Makaryus, 2021). On the right side of the heart, deoxygenated blood is pumped to the lungs where it perfuses into the lung tissue; on the left side of the heart, oxygenated blood is pumped to the rest of the body through the aorta. The smallest volume in the ventricles occurs after the ventricular repolarization which occurs during the $\mathrm{T}$ wave in the ECG.

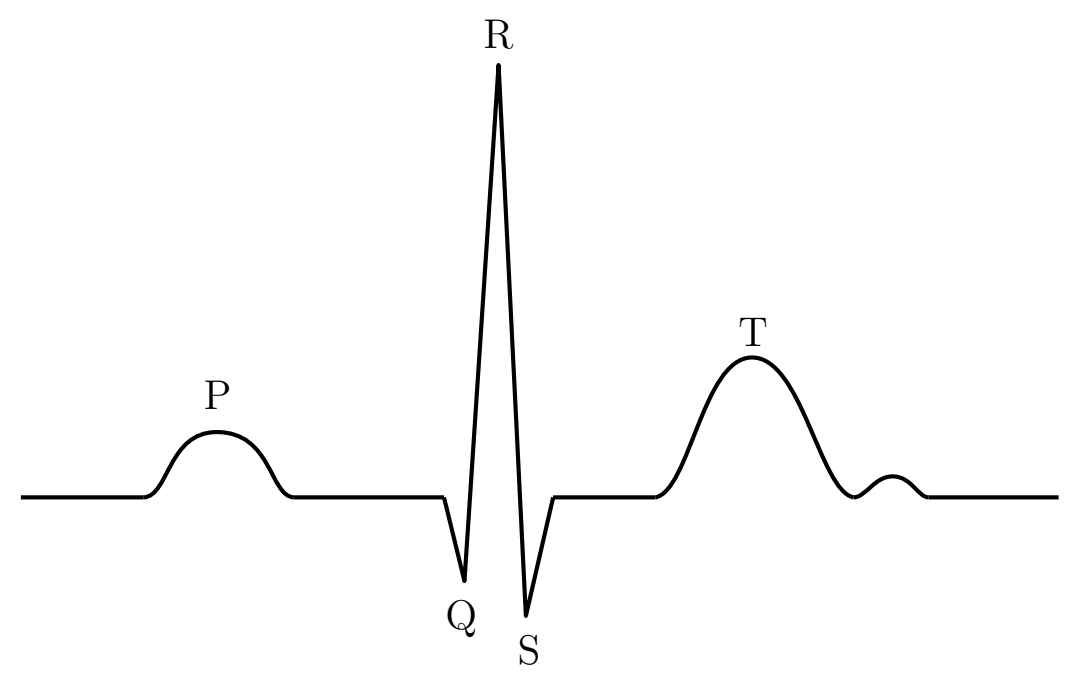

Figure 2.3: An example ECG waveform to compare electrical signals in the heart to blood volume changes. The $\mathrm{P}$ wave represents the beginning of the cardiac cycle, when the ventricles begin to fill. The QRS complex corresponds with ventricular depolarization and occurs as the ventricles contract. The beginning of the QRS complex corresponds with the maximum ventricular volume. The minimum volume in the ventricles coincides with the end of the $\mathrm{T}$ wave which represents ventricular repolarization. 


\subsubsection{Bioimpedance of Perfusion}

There are several factors that may contribute to some part of the impedance change during the cardiac cycle. Some of these factors include:

- Changes in blood volume within the heart as blood is pumped. As the ventricles fill with blood, the volume increases which results in a more conductive heart (Nyboer et al., 1970).

- Variations in arterial and blood vessel volume. Due to the elasticity of arteries and blood vessels, the pulsatile flow of blood passing through results in variation of vessel diameter, affecting the impedance (Eyüboğlu et al., 1987).

- Physical deformation of structures due to motion of the heart. The motion of the heart can have significant contribution to cardiosynchronous EIT images (Adler et al., 2017b), with simulations showing that heart motion was the main contributor to impedance change originating from ventricle (Proença et al., 2015).

- The orientation of red blood cells. During pulsatile flow, the orientation of red blood cells changes, which has been shown to affect the impedance of the blood (Gaw, 2010).

- Ballistic forces in the body generated by the heart. During each heartbeat, blood is pumped downwards through the descending aorta with a large force pushing the rest of the cardiac structures moving upwards (Gordon, 1877). Different directions of flow in the aorta result in a repeating ballistic signal on the rest of the body (Kim et al., 2016). This results in motion on the electrodes and body which can introduce significant artefacts in EIT signals (Adler et al., 
$1994)$.

The contribution of each of these factors will ultimately depend on the placement of the electrodes and the specific geometry and physiology of a patient. For example, when imaging changes in stroke volume, differences in posture, breathing and belt

position resulted in artefacts that overpowered the perfusion signals (Patterson et al., 2001).

Despite the challenges of isolating cardiosynchronous EIT signals related to perfusion, there is still a great interest in improving accuracy and stability of EIT for perfusion imaging. EIT is sensitivite to many different effects that may be representative of perfusion, and offers an advantage over many state-of-the-art methods due to its safety and portability. Increasing the sensitivity of EIT to cardiosynchronous changes could allow for a system that is able to continuously image perfusion at the bedside.

\subsection{Perfusion Imaging}

Determining the flow of blood within vital organs such as the heart, brain and lungs, allows doctors to make quick decisions to treat patients in life-threatening situations. In the brain, perfusion imaging can be used to detect ischemic strokes (Koenig et al., 1998; Konstas et al., 2009). or to help diagnose and monitor dementia and Alzheimer's disease (Barker et al., 2014, Dougall et al., 2004). Perfusion images of the heart can be used to diagnose ischemic heart disease (Prvulovich and Bomanji, 1998), which is caused by a reduction of blood flow to the heart, typically due to a build- 
up of plaque in the pulmonary arteries (Mendis et al., 2011). Ventilation/perfusion $(\mathrm{V} / \mathrm{Q})$ scans can be used in the lungs to measure the distribution of perfusion and ventilation in the lungs (Mortensen and Berg, 2019). The primary use of V/Q scans is to detect mismatches between the ventilation and perfusion to identify pulmonary embolisms (PIOPED-Investigators, 1990).

Nuclear medicine has been at the forefront of perfusion imaging, but has a long acquisition time, and images can be fuzzy and challenging to interpret. Recent developments in Computed tomography (CT) and magnetic resonance imaging (MRI) yield clearer images and can give metrics regarding blood flow and blood volume over a period of time. EIT has been proposed as a technique to monitor perfusion due to its sensitivity to blood movement, and development is currently underway to establish EIT as a technique to monitor perfusion (Nguyen et al., 2015 Nguyen et al., 2012), blood flow (Braun et al., 2018a; Braun et al., 2018b), and blood pressure (Proença et al., 2020, Proença et al., 2017). The following section explores the advantages and pitfalls of state-of-the-art perfusion monitoring techniques.

\subsubsection{Nuclear Medical Imagaing}

The three primary nuclear medical imaging techniques used for perfusion imaging are single-photon emission computed tomography (SPECT), scintigraphy, and positron emission tomography (PET). Both SPECT and scintigraphy image gamma rays that are emitted from a gamma-emitting radionuclitide injected into the patient (Mettler and Guiberteau, 2006). The radioisotopes are integrated into drugs that enable them to travel to a specific organ or tissue. Perfusion imaging with scintigraphy 
and SPECT is primarily used in the lungs and heart. The emitted gamma rays are measured by external detectors called gamma cameras (Mettler and Guiberteau, 2006). The main difference between the two is that SPECT creates 3D images, and scintigraphy gives 2D images. Scintigraphy uses a gamma camera placed in a single location, while SPECT uses a detector that rotates around the patient and captures gamma images from several angles. A typical SPECT system rotates 3-5 degrees per acquisition and takes approximately 15-20 minutes to complete a full scan. Scan times can be shortened by using multiple detectors to capture images from multiple angles simultaneously (Mettler and Guiberteau, 2006).

Nuclear medical imaging can be used to image both ventilation and perfusion, and has been used to diagnose pulmonary embolisms (Mortensen and Berg, 2019). Ventilation/perfusion scans are a specific type of perfusion scan that uses lung scintigraphy. During ventilation, an aerosolized radionuclitide is inhaled through a mask placed over the nose and mouth. To image lung perfusion, a different radionuclitide is injected and its passage through the lung is imaged. Both phases are imaged using a gamma camera (Mortensen and Berg, 2019). This technique can be timeand labour-intensive, and results in radiation exposure to both the technician and patient (Gandev et al., 2005). The duration of a single scan to measure ventilation and perfusion is approximately 15 minutes, including time required to breathe the radionuclitide prior to imaging (Hur et al., 2014). Reducing the time required to improve image acquisition time and reduce radiation exposure has been shown to give less reliable measures of perfusion (Hur et al., 2014).

PET relies on similar mechanisms to both SPECT and scintigraphy, but uses a 
different type of radiotracer. PET can be used to image myocardial perfusion using a radiotracer that emits positrons causing gamma rays to be emitted that are detected by gamma cameras. PET has not been used widely due to the higher cost of the system and radiotracers relative to SPECT (Driessen et al., 2017).

Nuclear medicine imaging methods have a lower radiation dose than CT perfusion imaging techniques, but suffer from a much lower resolution and longer imaging time (Aljizeeri et al., 2013). As such, CT is becoming the preferred method to image myocardial perfusion (Aljizeeri et al., 2013).

\subsubsection{CT}

Contrast-enhanced CT has been used since 1980 to estimate perfusion in the brain (Axel, 1980), and has more recently been used to image perfusion due to ischemic

stroke (Koenig et al., 1998, Miles et al., 1991). This technique uses an injection of 25-35 mL of a contrast agent, and the progress of the contrast agent through blood vessels and capillaries near to the brain is imaged over 75-90 seconds. Techniques are typically used to reduce overall radiation exposure, but imaging duration is limited by safety concerns due to radiation exposure (Konstas et al., 2009). Constrastenhanced CT has also been found to be an accurate and cost-effective technique to image myocardial perfusion in cardiovascular disease (Aljizeeri et al., 2013), but images require invasive cardiac catheterization and pose a risk of radiation exposure to both patients and technicians (Vijayalakshmi et al., 2007). 


\subsubsection{MRI}

There are three main techniques used to image perfusion with MRI. The first uses a contrast agent in the blood that decreases the intensity of images as the bolus passes (Jackson et al., 2005). The concentration of the contrast agent can be calculated using the image intensity drop, to give an approximation of perfusion (Jackson et al., 2005). The second technique also uses an injected contrast agent. This technique images the transit of a contrast agent as it travels from the blood into a tissue (Sourbron and Buckley, 2013). In this technique the contrast agent is not imaged directly, but the injected bolus impacts the magnetic properties of water molecules as it passes resulting in a change in image intensity in regions with contrast agent (Sourbron and Buckley, 2013). The final technique is arterial spin labelling, which does not rely on an injected contrast agent, but rather applies a magnetic label to water in the blood. Perfusion is then estimated by tracing the flow of this labelled blood (Koretsky, 2012).

Blood-oxygen-level-dependent imaging (BOLD-contrast imaging) uses blood flow to image functional activity (Faro and Mohamed, 2006). This technique compares the levels of oxygenated or deoxygenated blood that are present in the blood to estimate activity. Active neurons and tissues will have an increased oxygen consumption, resulting in a larger ratio of deoxygenated to oxygenated blood. This measure may also correspond to perfusion, but it does not directly image the flow of blood into the tissue.

MRI is typically used for cerebral imaging due to its safety and lack of ionizing radiation (Watson, 2015). It is not suitable for continuous perfusion imaging due 
to the acquisition time, which ranges from 1-5 minutes depending on the method (Essig et al., 2013). Due to the size and complexity of MRI imaging, it is also not a tool that can be used for bedside monitoring.

\subsubsection{EIT}

EIT has been suggested as an ideal tool to continuously monitor perfusion (Leonhardt and Lachmann, 2012). EIT is cost-effective, non-invasive, does not use ionizing radiation, and images can be acquired continuously at a high sampling rate (Adler and Boyle, 2017). Despite these advantages of EIT, spatial resolution is low and image accuracy is further limited by artefacts introduced by poor electrode contact and electrode movement (Adler and Boyle, 2017). The spatial resolution of EIT is typically thought to be approximately $20-50 \%$ of the electrode separation, but can be affected by the measurement protocol and image reconstruction techniques (Polydorides and McCann, 2002).

EIT has been evaluated for its ability to measure cardiac output and lung perfusion since the late 1980s (Brown et al., 1992 Eyüboğlu et al., 1989, Frerichs et al., 2002 Zadehkoochak et al., 1992). Since then, various configurations of EIT have been evaluated (Borges et al., 2012, Nguyen et al., 2015). Due to the speed and safety of measurement acquisition, EIT could be used to image perfusion in subjects much more frequently than current state-of-the-art techniques.

There are two main ways perfusion-related changes can be imaged with EIT. Like with many perfusion imaging techniques, a contrast agent can be injected to image the transit of blood through vessels and organs, or frequency filtering can be 
used to extract the portion of the signal related to perfusion. To image perfusion using a contrast agent a saline solution is typically used. As the concentration of ions in the blood increases, the conductivity increases proportionally creating measurable contrast (Solà et al., 2011). Typically a sodium chloride bolus with a concentration between $5-20 \%$ is used as a contrast agent for EIT (Nguyen et al., 2012). An algorithm must also be used to determine the regional blood flow to the lung and heart (Borges et al., 2012). This technique found that contrast agent-based measures of perfusion with EIT were highly correlated to SPECT images (Borges et al., 2012). The same study found that pulsatile images of EIT showed little correlation to bolus measures and SPECT scans.

When breathing is paused, the signal components related to cardiac activity can be more easily extracted. During apnoea the global impedance signal recorded with EIT has been found to correspond to stroke volume (Fagerberg et al., 2009). Ventilation perfusion ratios have been calculated during apnoea by comparing ventilation and perfusion signal amplitude with a specified region of interest (Fagerberg et al., 2009). There is some concern that the perfusion measured during apnoea may not accurately represent true perfusion during regular respiration as the apnoea impacts the regular respiratory and cardiovascular cycle (Leonhardt and Lachmann, 2012). Since apnoea cannot be sustained continuously, there is interest in an alternative method to increase sensitivity to cardiosynchronous changes.

The majority of work on perfusion imaging with EIT has focused on imaging the pulsatile or cardiosynchronous component of EIT data (Nguyen et al., 2012). As discussed in section section 2.2.2, there are many potential sources of cardiosynchronous 
signals, and their correlation with perfusion-related changes is not well understood. Perfusion had been calculated using digital filtering to extract the related component of an EIT signal. Deibele et al. (2008) have shown that principal component analysis (PCA) can be used to separate ventilation and cardiac frequency signals and identify the component related to the heart. Once the cardiac-frequency component of the EIT signal is identified, the pulmonary component must also be isolated. One of the most widely used techniques is selecting a region of interest that corresponds to the organ, tissue or flow to be measured (Braun et al., 2018a; Solà et al., 2011).

Techniques to improve accuracy of EIT models and increase sensitivity to cardiosynchronous activity, such as internal and 3D electrode configurations, could improve the accuracy of techniques to isolate pulsatile activity in a specific region, and could enable an improved perfusion measure. An improved measure of perfusion, with EIT based on filtering cardiosynchronous signals could also be used as a means of continuously monitoring perfusion during ventilation. This thesis explores the effect of both increased mesh accuracy and internal electrodes, and discusses their potential for improving sensitivity to perfusion with EIT. 


\subsection{Electrical Impedance Tomography}

Most EIT systems consist of: a current source to inject current between two electrodes; a system to measure differential voltages between pairs of electrodes; and electrodes, applied to the body surface. This section further discusses each of these aspects of EIT and introduces concepts of image acquisition and reconstruction.

\subsubsection{EIT Measurements}

A "frame" of EIT data consists of a series of measurements made on an array of electrodes placed around a region of interest. This thesis focuses on measurements of the thorax.

\subsubsection{Electrodes}

EIT recordings are made using several types of electrodes. Both polarizable and nonpolarizable electrode types are used; silver-silver chloride electrodes are often applied directly to the body, and stainless steel, brass, textile and rubber electrodes have also been used and are sometimes integrated into electrode belts. The polarization of EIT electrodes is not significant at the frequencies $(>20 \mathrm{kHz})$ that are typically used (Adler and Boyle, 2017).

When making measurements with EIT systems, there is very little current flowing through non-injecting electrodes due to the high impedance of the integrated voltmeter (Holder, 2004). For this reason, the effects of the electrode-body impedance are minimal on electrodes that are not injecting current. Measurements that are 
largely affected by this impedance on injecting electrodes typically have a phase shift and are often removed before reconstruction.

Changing contact impedance has a large effect on EIT measurements and can be influenced by changes in posture or pressure on electrodes (Coulombe et al., 2005). Contact impedance is often improved on electrodes by adding a conductive gel (Waldmann et al., 2017), but it can decrease over long measurements as the gel dries (Lozano et al., 1995).

\subsubsection{Current Injection and Voltage Measurement}

To calculate impedance, three main considerations must be made with regard to the injected current: the frequency, amplitude and injection pattern.

Low frequency currents will preferentially pass around cells through the extracellular fluid, due to the high capacitive component of the cell membrane (Foster and Lukaski, 1996), whereas high frequency currents will be more affected by the capacitive component (Holder, 2004). Since EIT requires a linear relationship between the measured voltages and tissue impedance to produce valid reconstructions, it is important to select frequencies of less than $1 \mathrm{MHz}$ to ensure that cell and neural membrane is not the dominant factor in the impedance measurements (Barber and Brown, 1984). Impedance measurements below $100 \mathrm{kHz}$ have shown to be primarily resistive (real) on lung tissue (Witsoe and Kinnen, 1967), and most EIT systems use frequencies between $10 \mathrm{kHz}$ and $1 \mathrm{Mhz}$ (Holder, 2004).

The selection of current amplitudes is primarily selected based on considerations to patient safety (Adler and Boyle, 2017). The guideline defined by IEC 60601-1 
(International Electrotechnical Commission, 2021), sets a limit of $10 \mathrm{~mA}$ on current across all electrodes at $100 \mathrm{kHz}$, and lower limits at lower frequencies. For EIT, frequencies between 20-200 kHz are often used with an injected current between 1-5 mA. In order to meet the safety guidelines at low frequencies, when injecting sinusoidal currents it is also important to use complete current cycles so that DC currents are not applied to the body (Adler and Boyle, 2017). When using internal electrodes, there are additional safety considerations that limit current amplitudes. Depending on the proximity to the heart, for clinical use it may be required to not inject current on internal electrodes. They can still be used to make measurements.

The pattern of current injection and voltage measurement is commonly referred to in EIT as the stimulation and measurement pattern. Typically a current is injected between two electrodes while the resulting voltages are measured between adjacent pairs. The two most common injection patterns are "adjacent", and "skip 4". Examples of these measurement patterns are shown in figure 2.4 on a 16-electrode system. The terms adjacent and skip 4 describe the space between pairs of injecting or measurement electrodes. Regardless of the pattern, a frame of EIT data is generated in the same way. Current is injected between a pair of electrodes as voltage is measured between all remaining pairs, then current is injected between the next pair of electrodes and voltages are measured again. This results in voltage measurements on each electrode pair for every current injection. For a 16-electrode system, this results in 256 measurements in each frame. For a 32-electrode system, there are 1024. As mentioned in section 2.4.1.1, voltage measurements made on the stimulating electrodes are often removed resulting in 208 measurements per frame on a 
16-electrode system, and 928 measurements per frame on a 32-electrode system.

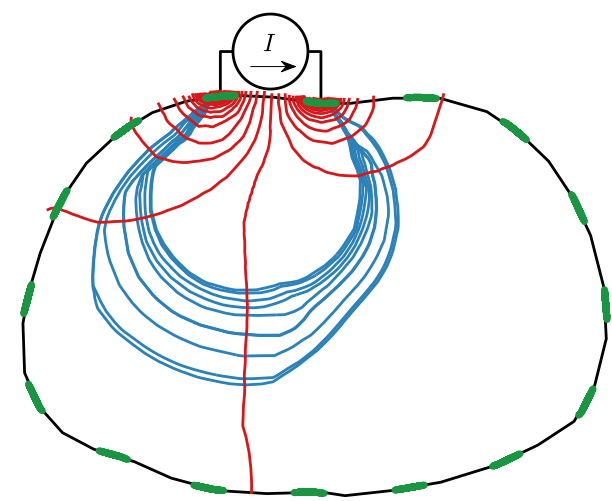

(A)

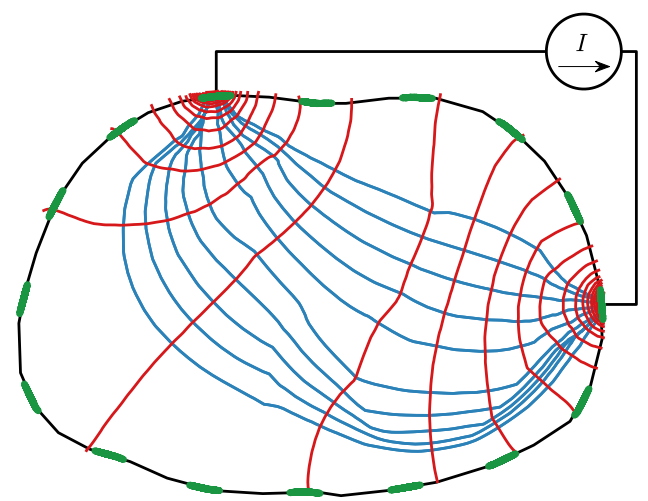

(B)

Figure 2.4: Two common stimulation patterns are simulated on a simple human model shown in figure 2.1. Injected current is shown in blue and the resulting equipotential lines are shown in red. (A) An example of an adjacent stimulation pattern. Current is injected between pairs of adjacent electrodes. For each current injection voltage is measured between all sequential pairs. (B) An example of the "skip 4" injection pattern. Current is injected between every $5^{\text {th }}$ electrode, skipping 4 between the injecting electrodes. During each current injection, voltage measurements are made using the same skip 4 pattern between each of the 16 electrodes and their corresponding pair. A single frame of data is generated by all measurements made for each possible injection pattern. For 16 electrodes, this results in 256 measurements.

\subsubsection{Imaging Techniques}

There are several challenges that make absolute EIT imaging difficult. The differences in tissue impedances are subtle relative to artefacts introduced by unknown boundary locations and electrode positions (Adler and Boyle, 2017; Adler et al., 2015; Nissinen et al., 2009). EIT is instead typically used to reconstruct impedance changes between two points in time.

Time difference EIT is sensitive to the movement of fluid within the body and 
is well suited to image functional activity, such as the inflation of the lungs and the flow of blood. Time-difference EIT is also much more stable in the presence of errors that remain constant, such as incorrectly modelled boundary and electrode locations (Brown, 2003). Frequency difference EIT is also possible based on the different impedance response of tissue types to changing frequencies. Frequency difference uses two or more different frequencies and calculates an image based on the change in electrical properties. Most frequencies used to differentiate between different tissue types are at high frequencies where current starts to flow across the cell membranes. These high frequencies are out of the range of most current EIT systems, and frequency changes due to different lower frequencies are limited (Adler and Boyle, 2017). This thesis uses time-difference EIT to image changes in fluid volumes and movement in the thorax.

\subsubsection{Forward Problem}

In simulation, typically we have a model with known geometry and impedance and we wish to solve for the voltage at the electrodes, due to a stimulation current. This

is called the forward problem. A model used to calculate a solution for the forward problem is called a forward model.

This problem is well-posed, and can be solved analytically on simple, regular geometries. For the forward solution, Maxwell's formulation of Faraday's Law is required:

$$
\nabla \times \vec{E}=-\frac{\partial}{\partial t} \vec{B}
$$


and Ampere's Law:

$$
\nabla \times \vec{H}=\vec{J}+\frac{\partial}{\partial t} \vec{D}
$$

To set up the solution we first assume that the current used for EIT is sufficiently low frequency relative to the scale of interest that we can use a quasistatic approximation of Maxwell's equations (Larsson, 2007). Under this assumption the derivative components of equation (2.1) and equation (2.2) can be set to zero giving:

$$
\nabla \times \vec{E}=0
$$

and

$$
\nabla \times \vec{H}=\vec{J}
$$

In this static case, the electric field due to a potential can be described by

$$
\vec{E}=-\nabla \vec{u}
$$

Finally, we require Ohm's Law, describing the relationship between the current density, conductivity and electric field:

$$
\vec{J}=\sigma \vec{E}
$$

Using the magnetic field identity for the divergence of $\operatorname{curl}(\nabla \cdot(\nabla \times \vec{H})=0)$ with equation (2.4), produces:

$$
\nabla \cdot \vec{J}=0
$$


Combining equation (2.7) with Ohm's Law (equation (2.6) gives:

$$
\nabla \cdot(\sigma \vec{E})=0
$$

And finally, using equation (2.5) relating the electric field and potential yields:

$$
\nabla \cdot \sigma \nabla \vec{u}=0
$$

Which gives the relationship between a conductivity distribution and the resulting potential.

The current density on the boundary is given by:

$$
j_{n}=-\vec{J} \cdot \vec{n}=\sigma \nabla \vec{u} \cdot \vec{n} .
$$

Where $\vec{n}$ is a unit vector normal to the boundary. Equation 2.10 cannot be used to specify current density at the boundary in EIT where the current density is not uniform (Somersalo et al., 1992). Instead we require a formulation that gives current density on injecting electrodes and no current density on the rest of the boundary. Somersalo et al. (1992) give a formulation of a complete electrode model for the voltage on $L$ electrodes $(E)$ where the voltage measured on each electrode $(U)$ is constant.

$$
u+z_{l}(\sigma \nabla u \cdot \vec{n})=U_{l} \quad \text { on } \quad E_{l}, \quad l=1,2, \cdots, L .
$$

The impedance of each electrode contact $(z)$ is also typically treated as a constant over the surface of an electrode (Somersalo et al., 1992). This complete electrode 
model has been shown to predict voltage accurately (Somersalo et al., 1992).

\subsubsection{Discretization and the Finite Element Method}

For EIT, analytic solutions are possible for simple geometries, but in a majority of cases numerical methods are required. One of the primary discretization techniques is the finite element method FEM where the domain is divided into a number of smaller elements. An element is triangular in 2D and tetrahedral in 3D. The collection of nodes, and elements making up a larger model is commonly called a mesh. Each line connecting two nodes of a mesh can be visualized as a resistor, used to model the conductivity of an object.

To accurateatly solve numerical problems using the FEM, it is vital to accurately represent spatial information. Boyle and Adler (2011) show that incorrectly modelling the boundary and electrodes can result in distorted images. Especially in brain EIT, great care is taken to minimize errors in the forward model (Aristovich et al., 2014; Malone et al., 2014), as small errors in modelling have been shown to give large errors in the reconstructed images (Kolehmainen et al., 1997).

\subsubsection{Inverse Problem}

To reconstruct images we must solve for impedance distribution from known voltage measurements. This is known as the inverse problem. The ability to reconstruct impedance distributions from a known voltage distribution is challenging and relies on the assumption that the relationship between voltage and conductivity is linear (Barber and Brown, 1984). Unlike the forward problem, due to the small number 
of electrodes relative to the large number of internal elements the inverse problem is ill-posed (Holder, 2004).

The sensitivity of EIT is often represented by a jacobian matrix, $\mathbf{J}$, which gives the change in measurement relative to another factor. To reconstruct a conductivity distribution, a conductivity jacobian, $\mathbf{J}_{C}$, is used which links the change in boundary voltage measurements $(U)$ to the change in impedance $(\sigma)$. It can be represented by

$$
\left[\mathbf{J}_{C}\right]_{i j}=\frac{\partial U_{i}}{\partial \sigma_{j}}
$$

The jacobian gives the relationship between the conductivity of each voxel and the resulting measurements (Holder, 2004). In practice the jacobian can be calculated using the adjoint field (Vauhkonen et al., 1999). The formulation of sensitivity gives

rise to the units of $\frac{\Delta \mathrm{V}}{\Delta \frac{\mathrm{S}}{\mathrm{m}}}$. Errors in sensitivity calculation can negatively impact the reconstruction. Errors in regions of high sensitivity near the electrodes are especially problematic as they will propagate affecting regions with low sensitivity as well. For this reason it is important to correctly calculate the sensitivity matrix, or jacobian, especially in regions of high sensitivity.

\subsubsection{Regularization}

The sensitivity of measurements to changes in conductivity is vastly different throughout a model. Near electrodes, where the current density is high, there is high sensitivity to conductivity changes but there is limited sensitivity away from the electrodes. This contributes to the ill-posed nature of the inverse problem (Holder, 2004). To overcome this regularization must be used to stabilize reconstruction. 
Regularization imposes restrictions on a minimization problem to ensure a unique solution. In EIT, some typical regularization techniques include the maximum a priori approach (Adler and Guardo, 1996), Tikhonov regularization (Vauhkonen et al., 1998), and singular value decomposition (Ostebee, 1998).

\subsubsection{Image Reconstruction}

The standard linear formulation (Holder, 2004) to solve for the conductivity change in each voxel $(\Delta \hat{\sigma})$ for a given set of difference measurements $(\mathbf{b})$ is

$$
\Delta \hat{\sigma}=\mathbf{J}^{T} \boldsymbol{\Sigma}_{n}\left(\mathbf{J} \boldsymbol{\Sigma}_{p} \mathbf{J}^{T}+\boldsymbol{\Sigma}_{p}\right)^{-1} \mathbf{b}
$$

Where $\Sigma_{n}$ and $\boldsymbol{\Sigma}_{p}$ are covariance matrices that incorporate a priori information. $\Sigma_{n}$ represents covariance in the data space and weights noise contribution to reconstructed images, and $\boldsymbol{\Sigma}_{n}$ represents covariance in the image space, and controls regularization (Adler and Guardo, 1996). The term $\mathbf{J}^{T} \boldsymbol{\Sigma}_{n}\left(\mathbf{J} \boldsymbol{\Sigma}_{p} \mathbf{J}^{T}+\boldsymbol{\Sigma}_{p}\right)^{-1}$ is also called the reconstruction matrix and can be represented by the term $\mathbf{R}$, since after initial calculation it typically remains constant for a given model. Conductivity distributions can be solved using the reconstruction matrix using the equation:

$$
\Delta \hat{\sigma}=\mathbf{R b}
$$

In reconstructed EIT images, the conductivity is typically represented in arbitrary units of $\Delta \mathrm{Z}$ representing a change in impedance. Due to the reconstruction algorithms, it is not feasible to calculate the units of conductivity from the reconstructed 
image.

There are several techniques used to calculate error weighting and prior information represented by $\Sigma_{n}$ and $\boldsymbol{\Sigma}_{p}$, such as the NOSER algorithm (Cheney et al., 1990). There are also techniques that have been specifically designed to compensate for the limitations and characteristics of EIT systems such as GREIT (Adler et al., 2009).

\subsubsection{Image Reconstruction With GREIT}

GREIT (Graz consensus Reconstruction algorithm for EIT) is a consensus algorithm that is used to reconstruct EIT images according to limitations and characteristics of typical EIT systems (Adler et al., 2009). The reconstruction matrix calculated with GREIT depends on the forward model, noise model and the desired performance metrics. GREIT uses a forward model that models electrodes using the complete electrode model discussed in section 2.4.3. The forward model has information regarding the stimulation measurement pattern, body geometry, and electrodes.

GREIT constructs a set of training images using a small (less than $5 \%$ of the model diameter) conductive target in the forward model. A training set consists of images of a conductive object placed at many (100-500) locations within the model. A noise model consisting of a Gaussian estimate of measurement noise, and measurements with electrode motion noise is also generated.

Training targets are blurred so that their diameter increases to $20 \%$ of the model diameter, but the position remains constant. This is used as the desired image for a training target. The weighting relative to noise and other performance metrics is set to minimize reconstruction errors between the training target and ideal image. The 
weights are set based on the radius of the training image to penalize changes outside the desired image radius and minimize shape deformation and ringing artefact. The weighting can also be adjusted to meet specific performance characteristics of interest. A description of all performance characteristics that can be specified is found by Adler et al. (2009). When training the GREIT algorithm, weighting is also set to increase uniformity of the resolution in the reconstructed image. Some resolution near the electrode is lost, but weighting helps to ensure that targets reconstruct to the same size throughout the model.

To calculate a reconstruction matrix $(\mathbf{R})$, error $(\epsilon)$ is minimized over all training targets $(k)$ with respect to the weighting parameters $\left(\mathbf{w}^{(k)}\right)$, measurements $(\mathbf{b})$, and the desired images $(\tilde{\mathbf{x}})$.

$$
\epsilon^{2}=\sum_{k}\left\|\tilde{\mathbf{x}}^{(k)}-\mathbf{R b}^{(k)}\right\|_{\mathbf{w}^{(k)}}^{2}
$$

To minimize the equation for error, the derivative of $\epsilon^{2}$ with respect to the reconstruction matrix is set to 0 .

$$
\epsilon^{2}=\sum_{k}\left\|\tilde{\mathbf{x}}^{(k)}-\mathbf{R b}^{(k)}\right\|_{\mathbf{W}^{(k)}}^{2}
$$

Grychtol et al. (2016) show that equation 2.15) can be rearranged to solve for the reconstruction matrix as:

$$
\mathbf{R}=\mathbf{D} \boldsymbol{\Sigma}_{p} \mathbf{J}^{T}\left(\mathbf{J} \boldsymbol{\Sigma}_{p} \mathbf{J}^{T}+\lambda \boldsymbol{\Sigma}_{n}\right)^{-1}
$$


Where $\mathbf{D}$ is the desired image metric from all training images, $\boldsymbol{\Sigma}_{p}$ is the effective covariance of all training targets after weighting with $\mathbf{W}$, and $\boldsymbol{\Sigma}_{n}$ is the noise covariance. This reconstruction method is widely used in EIT for both $2 \mathrm{D}$ and $3 \mathrm{D}$ electrode configurations and is shown to give even resolution throughout the image (Adler et al., 2009).

\subsubsection{Internal Electrodes}

While there have been some studies researching electrode placement for cardiac imaging in 2D (Vonk Noordegraaf et al., 1996) and 3D electrode configurations (Graham and Adler, 2007), there has been little research into the application of internal electrodes. It has been shown that externally placed 3D electrode configurations consisting of two electrode planes can improve the sensitivity distribution and image quality (Grychtol et al., 2016), but there is still limited sensitivity in the central-most regions of the chest. The concept of using internal esophageal reference electrodes has been presented previously (Pilkington et al., 1989; Schuessler and Bates, 1995) as a method to improve internal sensitivity, and has shown accuracy improvements of up to $30 \%$ for small targets (Pilkington et al., 1989). Recent simulations in 2D have shown an increase in reconstruction accuracy, amplitude response, position error, and resolution (Nasehi Tehrani et al., 2012a; Nasehi Tehrani et al., 2012b).

A clinical evaluation of EIT found that the amplitude of cardiosynchronous signals in EIT measurements was 6 times larger when placing 2 of 16 total electrodes in either the esophagus or trachea, but found that reconstructions in an animal model were not obviously improved in all cases (Czaplik et al., 2014). A recent review 
highlighted the use of internal electrodes to monitor cardiac radiofrequency ablation; investigate the possible noise sources; and stated the need for analysis of the effect of motion on the internal electrodes (Nguyen et al., 2020).

Despite several promising simulation results using internal electrodes, the clinical advantage has not been realized. Internal electrodes are a challenging problem. Simulation studies in 2D have shown that internal electrodes should improve sensitivity, but there have been no studies that show these improvements in reconstruction accuracy in-vivo. Conversations with colleagues suggest that the high sensitivity near the internal probe it one of the causes of this issue, and recently published work has suggested that movement of the internal probe may contribute significantly to this error (Nguyen et al., 2020). Some other factors that may contribute to the error using internal electrodes are the imbalance of sensitivity that worsens the ill-posed nature of EIT image reconstruction and contact of the internal electrodes on the esophagus. Chapter 7 aims to determine the contribution of motion of the internal probe of on reconstructions using internal electrodes and presents a technique to correct for error introduced by motion of the internal probe. This thesis presents uses this correction technique to generate reconstructions of ventilation in-vivo, and compares currently available reconstruction algorithms.

While studies show an increased sensitivity to cardiosynchronous EIT signals, the source of these signals is not clear, and the impact of movement on the internal electrodes is not known. 


\subsection{Summary}

EIT has potential to be used as a tool for continuous perfusion monitoring at the bedside, but is limited by low sensitivity to cardiosynchronous signal. Accurate modelling and the use of internal electrodes have been proposed as techniques to increase the sensitivity of EIT in specific regions, but it is not clear how much these might improve perfusion imaging. We also examine the effect of internal electrode movement and potential methods to correct these errors. Improving sensitivity to cardiosynchronous signals with EIT could enable better isolation of the perfusion related signal from other factors, and improve the use of EIT for cardiovascular monitoring. 


\section{Chapter 3}

\section{Bolus- and Frequency-Based Perfusion}

This chapter has been adapted from:

Stowe, S., Boyle, A., Sage, M., See, W., Praud, J.-P., Fortin-Pellerin, É., 6 Adler, A. (2019). Comparison of bolus-and filtering-based EIT measures of lung perfusion in an animal model [Publisher: IOP Publishing]. Physiological Measurement, 40(5), 054002

\subsection{Introduction}

There are two main imaging techniques used to image perfusion with EIT: bolus injection, and frequency filtering. This chapter compares bolus-based and filteringbased measures of perfusion in an effort to improve understanding of the different sources of cardiosynchronous signal. Filtering-based measures of perfusion are ap- 
pealing since they are less invasive and can be used continuously, but the source of these signals is controversial and it is not clear how accurately they represent perfusion (Adler et al., 2017b). Determining how filtering-based techniques compare to contrast agent techniques could help to determine if the cardiosynchronous signals can be used to monitor perfusion non-invasively.

Electrical Impedance Tomography (EIT) uses electrical stimulation and measurements at electrodes on the body surface to reconstruct images of an internal conductivity distribution and its changes. The most common application of EIT, experimentally and clinically, has been for imaging of the thorax (Frerichs et al., 2017). Using a ring of electrodes around the chest, EIT calculates images of impedance changes in the abdomen. Although most research has focused on imaging ventilation, there is significant interest in imaging cardiovascular phenomena with EIT (Adler et al., 2012; Leonhardt and Lachmann, 2012).

EIT has been evaluated for its ability to measure cardiac output and lung perfusion since the early 90s (Eyüboğlu et al., 1989 Frerichs et al., 2002 Zadehkoochak et al., 1992). Since then, various configurations of EIT have been evaluated (Borges et al., 2012 Nguyen et al., 2015). The effect of posture on EIT images was evaluated by Reifferscheid et al. (2011), who showed that changing posture introduces a large and reproducible variability into ventilation distribution as imaged by EIT. Based on results showing a common relationship between the effect of gravity and perfusion in both children and adults (Bhuyan et al., 1989), we expect to see a comparable directional change in perfusion due to postural changes newborns. Recently, Braun et al. (2018) evaluated EIT's ability to monitor cardiac output, showing that EIT 
is more reliable for monitoring cardiac output trends than absolute cardiac output. EIT has also been investigated for monitoring systemic blood pressure (Solà et al., 2011) and pulmonary arterial pressure (Proença et al., 2017).

EIT measurements are sensitive to blood movement in two main ways. First, it is possible to image the transit of the contrast agent through the heart and lungs via a conductivity-contrasting bolus into the veins. Second, digital filtering of the time series of EIT images at the heart frequency can be used to extract the perfusion related signal (Leathard et al., 1994). While multiple EIT measures of perfusion are used, their relationship is not well understood. It is currently unclear to what degree pulsatile impedance changes represent blood flow, and how this limits the potential for heart-frequency filtering to correctly estimate the true perfusion (Nguyen et al., 2012).

Injection of a contrast agent to measure regional lung perfusion has been compared with electron beam computed tomography (EBCT) and determined to be feasible for measuring perfusion across different animals (Frerichs et al., 2002). Perfusion measurement via contrast agent has the advantage of imaging true perfusion, but requires placement of a catheter to introduce the conductive agent. Bolus-derived measurements cannot be made continuously because they rely upon the circulation of a contrast agent. In addition, the accumulation of sodium chloride $(\mathrm{NaCl}-$ the main conductivity contrast used for EIT imaging) over multiple injections can lead to hypernatremia which limits the rate at which bolus injections can be made.

Calculating the heart-frequency conductivity changes in the thorax offers the benefit of a continuous functional measure calculated directly from EIT signals (possibly 
in conjunction with a synchronization signal such as the ECG). Heart-frequency EIT signals are typically an order of magnitude smaller than ventilation signals. Thus, when measurements are made during tidal ventilation, a large period of data must be used in order to remove the ventilation signal. Measurements during apnoea can be used to eliminate the ventilation signal, but for the safety of the patient the apnoea duration should be limited. In healthy human subject of less than one year old, it takes a mean of $118 \mathrm{~s}$ for the blood oxygen saturation levels to drop below $90 \%(\mathrm{Fu}$ et al., 1996). However, the length of safe apnoea is much shorter for the sick preterm infant. We selected an apnoea duration of 30 seconds since based on experience in the lab it was not associated with bradycardia or desaturation to less than $90 \%$ blood oxygen saturation.

There is a debate within the EIT community about the meaning of heartfrequency EIT signals (Adler and Boyle, 2017; Frerichs et al., 2017). Not all perfusion results in a cardiac-frequency change (for example, continuous blood flow in capillaries), and non-perfusion effects (for example, heart movement in the thoracic cavity) can result in heart-frequency EIT signals. This debate is reflected by the terminology - perfusion vs. pulsatility. Those who prefer "pulsatility" or "heart-frequency fEIT image" seek to emphasize that frequency filtered signals are not "perfusion" (although they may be related). While these pulsatility based EIT images are clearly not a direct measure of perfusion, the signals appear to be useful and are often measured and reported (Bartocci et al., 1999; Ericsson et al., 2016, Halter et al., 2008; Moens et al., 2014). To the authors' knowledge, no systematic comparison of frequency-based perfusion measures has been published. 
The heart-frequency signal can be derived from frequency filtering or ensemble averaging. Frequency-filtering uses a filter to isolate the frequency of heartfrequency conductivity changes, and was introduced by Zadehkoochak et al. (1992) and Leathard et al. (1994). Frequency filtering is susceptible to interference from ventilation when the heart rate is at a harmonic of the breathing rate. Ensemble averaging is another filtering approach which averages signals at a synchronized time, for example at the QRS peak (Bartocci et al., 1999; Deibele et al., 2008). The impedance change due to each heart beat is aligned and averaged to give a single heart-related impedance change, representative of all heartbeats in the segment.

In this paper, we are motivated to better understand the relationship between lung perfusion and heart-frequency filtering measures, and between the various filtering approaches used to determine heart-frequency components. Our questions are: 1) to what extent do heart-frequency filtering-based measures correspond to perfusion, 2) what are the advantages and disadvantages of different approaches to heart-frequency filtering of EIT data, and 3) which techniques are recommended. In our experimental protocol, we have selected posture change to introduce changes in the regional distribution of lung perfusion. These changes are then compared using bolus- and filtering-based EIT measures. 


\subsection{Methods}

\subsubsection{Overview}

Data were acquired as an additional protocol within a study to determine a baseline for lung damage due to gas ventilation in neonatal lambs. This is part of an effort to establish total liquid ventilation (TLV) as a less injurious ventilation strategy for the delicate lungs of neonatal subjects (Sage et al., 2018). In order to induce changes in ventilation and perfusion patterns, posture changes were made between supine, prone, left and right lateral positions.

\subsubsection{Animals}

The study was conducted in accordance with the Canadian Council on Animal Care guidelines upon approval by the animal research ethics board of Université de Sherbrooke (protocol 417-17BR).

Seven healthy neonatal lambs (2-4 days old and $2.95 \pm 0.27 \mathrm{~kg})$ were used. Animals were anaesthetized (ketamine $10 \mathrm{mg} / \mathrm{kg}$ IM at induction followed by propofol $100 \mathrm{mcg} / \mathrm{kg} / \mathrm{min}$ and ketamine $2 \mathrm{mg} / \mathrm{kg} / \mathrm{h} \mathrm{IV}$ ) and placed under mechanical gas ventilation with peak inspiratory pressure (PIP) $15 \mathrm{cmH}_{2} \mathrm{O}$, positive end-expiratory pressure (PEEP) $5 \mathrm{cmH}_{2} \mathrm{O}$, respiratory rate (RR) of $60 / \mathrm{min}$, and fractional concentration of $\mathrm{O}_{2}$ in inspired gas $\left(\mathrm{FiO}_{2}\right)$ of $30 \%$.

A catheter was inserted into the carotid artery for blood gas and continuous blood pressure monitoring. A jugular venous access was inserted to inject the saline bolus for generating perfusion images. Each animal was shaved for placement of a custom 
EIT belt around the lower third of the sternum in the transverse plane.

For each animal a bolus injection protocol was used: $1.5 \mathrm{~mL}$ of $7.5 \%$ saline was injected into the jugular vein at a constant rate over approximately 2 seconds. Before each bolus, ventilation was stopped for 10 seconds, and a further 20 seconds of apnoea was maintained before restarting ventilation.

After one hour of ventilation (for stabilization) EIT recordings were made during the position change procedure. Each lamb was rotated onto its right side. Five minutes after turning the subject, the bolus injection protocol was implemented. The animal was then ventilated normally, remaining on the right side for an additional 5 minutes, before being positioned on the left side for 5 minutes of regular ventilation. Five minutes after each posture change the bolus injection protocol was performed. After 2 hours of ventilation, the position change procedure was repeated, changing the positioning of the lamb from prone to supine as the bolus injection protocol was repeated and EIT recordings were captured. 


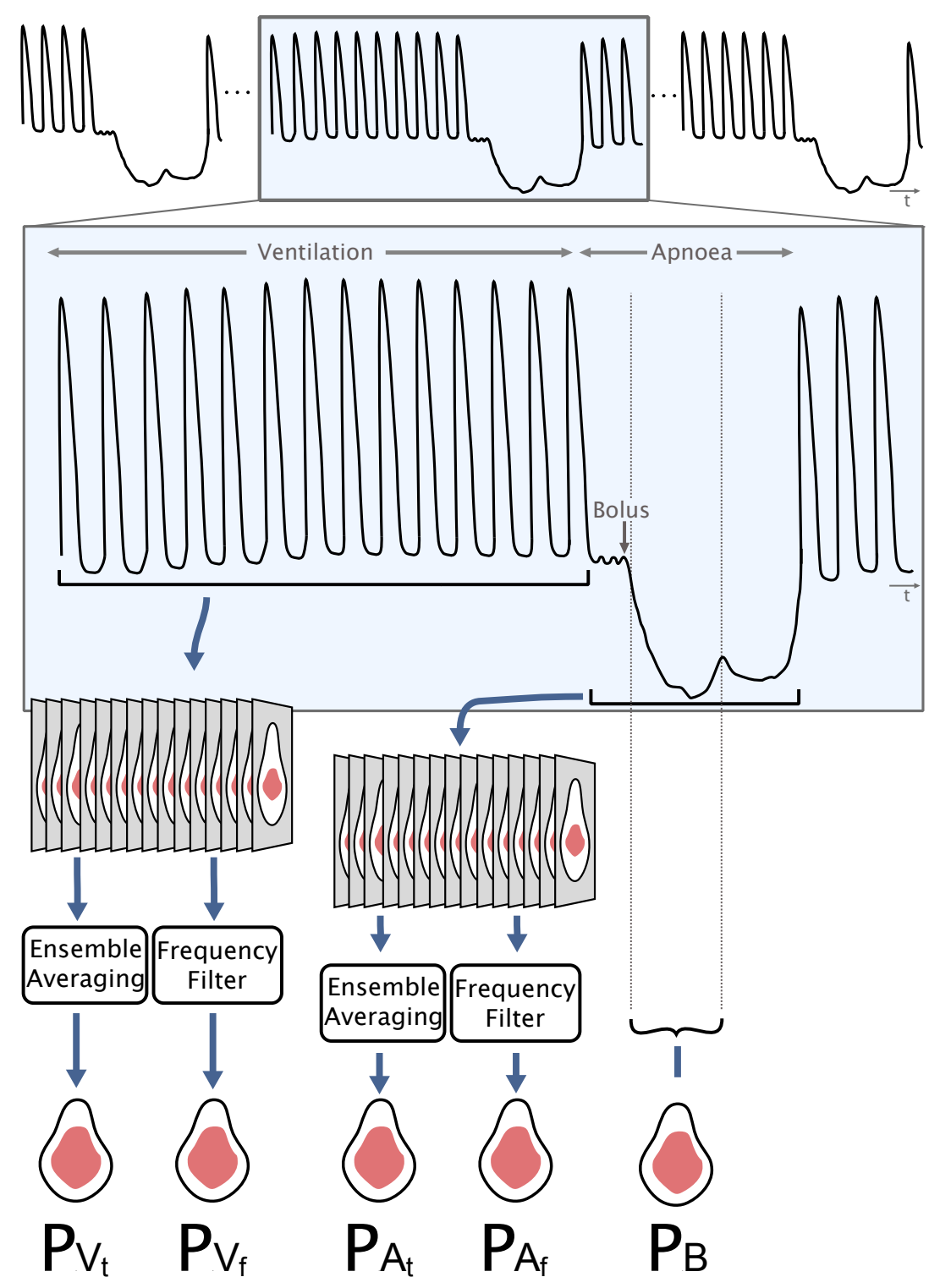

Figure 3.1: This figure is a schematic overview of analysis methods for EIT perfusion. The upper curve illustrates the global EIT signal during a period of ventilation followed by apnoea and renewed ventilation. During apnoea a bolus of conductivity contrasting saline is introduced. From these data 5 fEIT images are calculated: $\mathrm{P}_{\mathrm{Vt}}$ : pulsatility (perfusion) image during ventilation, calculated by ensemble averaging EIT data during ventilation; $\mathrm{P}_{\mathrm{Vf}}$ : pulsatility (perfusion) image during ventilation calculated by frequency filtering EIT data during ventilation; $\mathrm{P}_{\mathrm{At}}$ : pulsatility (perfusion) image during apnoea calculated by ensemble averaging EIT data during apnoea; $\mathrm{P}_{\mathrm{Af}}$ : pulsatility (perfusion) image during apnoea calculated by frequency filtering EIT data during apnoea; $\mathrm{P}_{\mathrm{B}}$ : perfusion image from bolus calculated between a reference measure during apnoea and one during the bolus 


\subsubsection{Data Acquisition and Image Reconstruction}

EIT data was acquired with the Pioneer Set (Swisstom, Landquart, Switzerland) using a custom electrode belt (at an acquisition rate of 20 frames/s). The belt uses 32 brass electrodes equally spaced around the thorax, ultrasound gel was applied to the electrode surface prior to application to ensure good contact and minimize the contact impedance. The selected data in this study comes from lateral positioning changes recorded after 1.5 hours of ventilation and prone to supine positioning changes after 2 hours.

EIT images were reconstructed using GREIT (Adler et al., 2009), which calculates a reconstruction matrix $\mathbf{R}$ from which the reconstructed image is calculated as $\hat{\mathbf{x}}=$ $\mathbf{R y}$, where $\mathbf{y}$ are the time-difference measurements, $\mathbf{y}(t)=\mathbf{v}(t)-\mathbf{v}\left(t_{r}\right)$, where $\mathbf{v}(t)$ represents the data frame acquired at time, $t$, and $\mathbf{v}\left(t_{r}\right)$ measurements acquired at a "reference" time, $t_{r}$ in the case of this experiment the reference was a mean of 10 images preceding the bolus injection.

The linear reconstruction matrix $\mathbf{R}=\mathbf{D} \boldsymbol{\Sigma}_{t} \mathbf{J}^{T}\left(\mathbf{J} \boldsymbol{\Sigma}_{t} \mathbf{J}+\boldsymbol{\Sigma}_{n}\right)^{-1}$ is calculated from a finite element model of the body and electrode geometry $F(\cdot)$ and covariance estimates of the image, $\boldsymbol{\Sigma}_{t}$, noise, $\boldsymbol{\Sigma}_{n}$ (Grychtol et al., 2016), and a spatial filtering matrix, D.

EIT data from this experiment was prone to errors consisting of brief periods of zeroed measurements. Measurements that were zeroed by the device were removed and replaced with linearly extrapolated data to allow for frequency-based analysis overall selected segments of data. A moving median filter with a width of 3 was used to further remove the noise caused by single measurement errors in the signal. 


\subsubsection{Functional EIT Images}

In each animal 4 episodes were recorded - one in each posture - to generate 5 different functional EIT images.

The images Bolus-based measures of lung perfusion $\left(\mathrm{P}_{\mathrm{B}}\right)$ were calculated using time-difference reconstructions. Heart-frequency filtering during ventilation $\left(\mathrm{P}_{\mathrm{Vf}}\right)$ and apnoea $\left(\mathrm{P}_{\mathrm{Af}}\right)$ used frequency analysis of EIT image sequences, as illustrated in figure 3.3 , and ensemble averaging-based methods during ventilation $\mathrm{P}_{\mathrm{Vt}}$ and apnoea $\mathrm{P}_{\text {At }}$ are calculated using ensemble averaging of identified pulsatile components figure 3.4

The following methods were conducted on segments of data collected both during apnoea and ventilation. Apnoea regions were selected as the total time that ventilation was arrested, including the bolus section and had a duration of 30 seconds. The ventilation data was selected as 30 seconds of data immediately preceding the induction of apnoea. Regions of interest including lung, and heart areas in the images were defined by the lamb model provided in EIDORS (Adler et al., 2017a).

\subsubsection{Bolus Injection Image $\left(P_{B}\right)$}

The beginning of the saline bolus injection was determined as the point immediately preceding the drop in impedance from the conductive agent, and is shown in figure 3.2 at the point marked "injection." The mean of 10 images including and immediately preceding the bolus injection were used as the reference to which all bolus images were reconstructed from. To image perfusion, the point with maximum decline in impedance over the sum of the pixels in the lung region relative to the reference was 
selected based on the methods presented by Frerichs et al. (2002). In figure 3.2 this was found at the point marked "perfusion." This method was used as the standard perfusion measuring technique against which the other methods were compared.

\section{Apnoea}

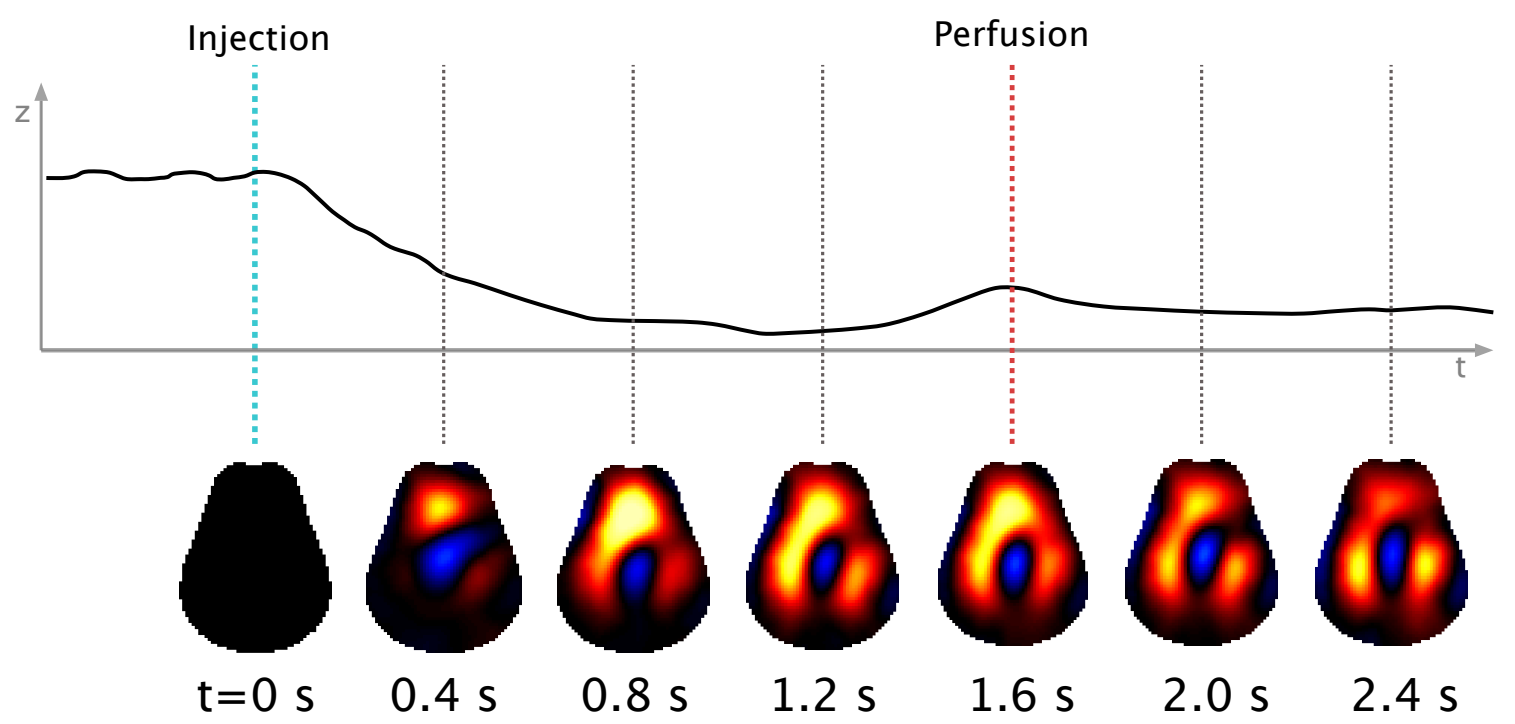

Figure 3.2: $\quad$ The method used to select the perfusion point from the bolus injection is shown in the figure above. The point with the widest spread of high conductivity was selected as the point of perfusion, shown here at 1.6 seconds after the contrast agent injection. The image series shows the conductivity contrast as the bolus injection travels through the thorax.

\subsubsection{Frequency-Filtering}

Heart-frequency EIT images during the selected events were calculated by taking the FFT of the time-series image data after first applying a Blackman window: $w(n)=a_{0}-a_{1} \cos \left(\frac{2 \pi n}{N-1}\right)+a_{2} \cos \left(\frac{4 \pi n}{N-1}\right)$ with $a_{0}=0.42, a_{1}=0.5$ and $a_{2}=0.08$, where $\mathrm{N}$ is the number of time-series EIT images in the selected event.

An FFT was calculated from a series of images restricted to pixels in the heart 
region. From the FFT of all pixels the heart region, the heart frequency was selected as the largest peak between 3 and $4.5 \mathrm{~Hz}$, representing a heart rate between 180 and 240 bpm (typical for a newborn lamb).

The identified heart rate was used to select changes at the heart-frequency in the frequency domain images of the entire thorax. Images at 3 frequencies on either side of the heart rate were also reconstructed to account for changed in heart rate over the course of the data collection. A Blackman window with a length of 7 was applied surrounding the heart frequency to generate a weighted mean of the images, resulting in a single perfusion image from the heart-frequency data.

The output of the frequency filtering method is an image with complex values assigned to each pixel.

Depending on the timing of the pulsatility-based changes within the selected signal the real component of frequency analyzed image did not correspond to the maximum conductivity change in the lungs in every event. In order to correct this, each image was displayed along the axis that gave the maximum real component contained within the lung region to ensure the maximum change in impedance related to pulsatile activity in the lungs was calculated. 


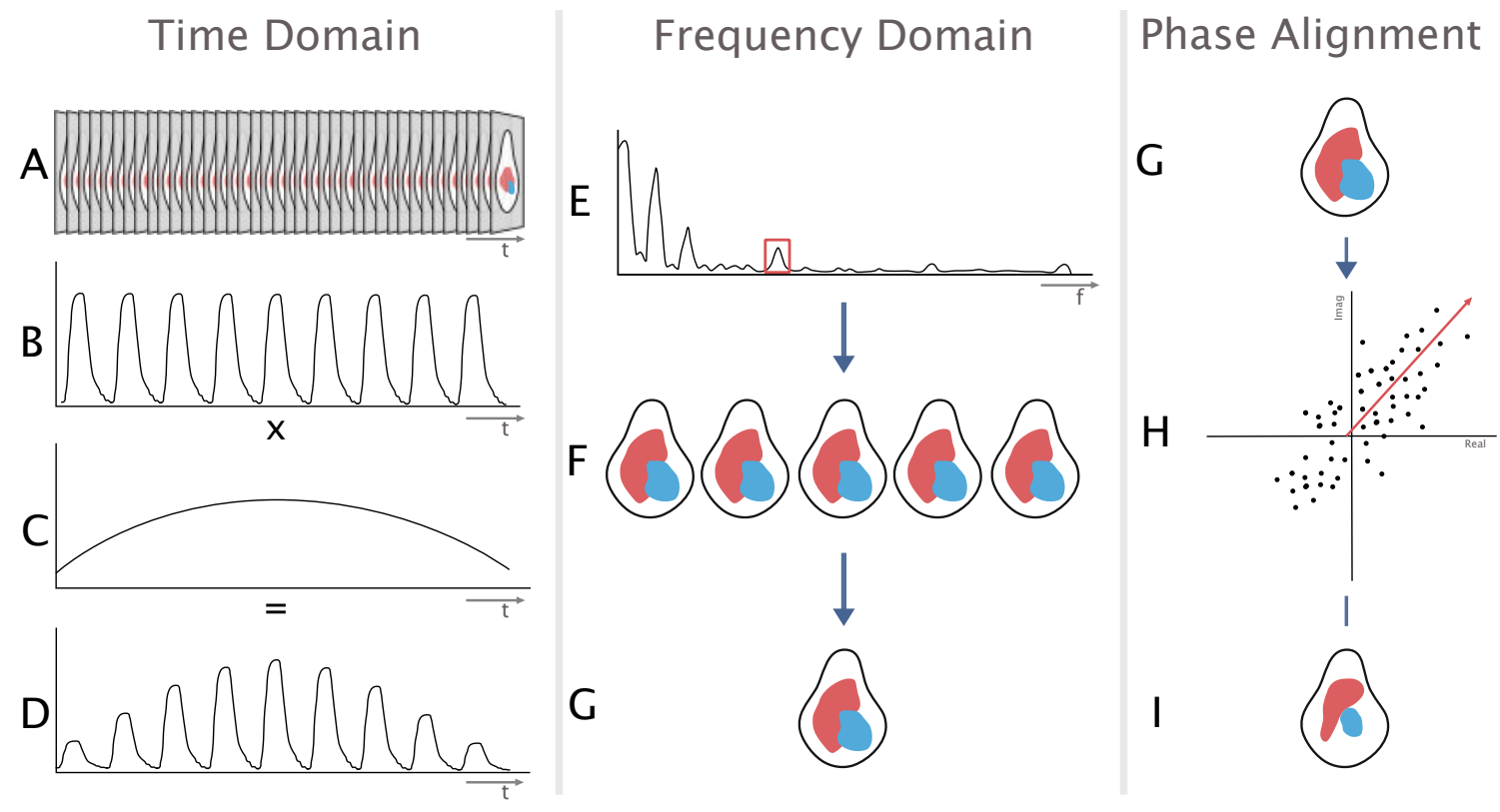

Figure 3.3: Frequency analysis methodology used for obtaining a perfusion image from the time series data. Steps are: A) to reconstruct the images from time series measurements; B) - D) window the time series data before performing a FFT on the data for each element; E) Select the dominant frequency between 3 and $4.5 \mathrm{~Hz}$ as the heart frequency; F) reconstruct the image at the heart frequency and selected nearby frequencies; G) take the mean of the images at the heart frequency using a Blackman window to give greater weight to those closer to the centre; H) I) select the image that will give the maximum real component contained in the lung region.

\subsubsection{Ensemble Averaging}

Time series data of the total impedance signal for each pixel in the heart region was filtered using a bandpass filter to eliminate noise and breathing changes, and allow the heartbeat to be seen clearly in the signal. Peak detection was used on this heart-region data to select the amplitude peaks in impedance change signal at the heart frequency.

Using the identified time points, the global impedance change signal was ensemble 
averaged by overlaying all identified peaks to give an averaged heartbeat. Thirteen images were reconstructed over the course of the heart beat to select the image that resulted in the maximum positive increase impedance within the lung region. This process is outlined in figure 3.4 .

A)

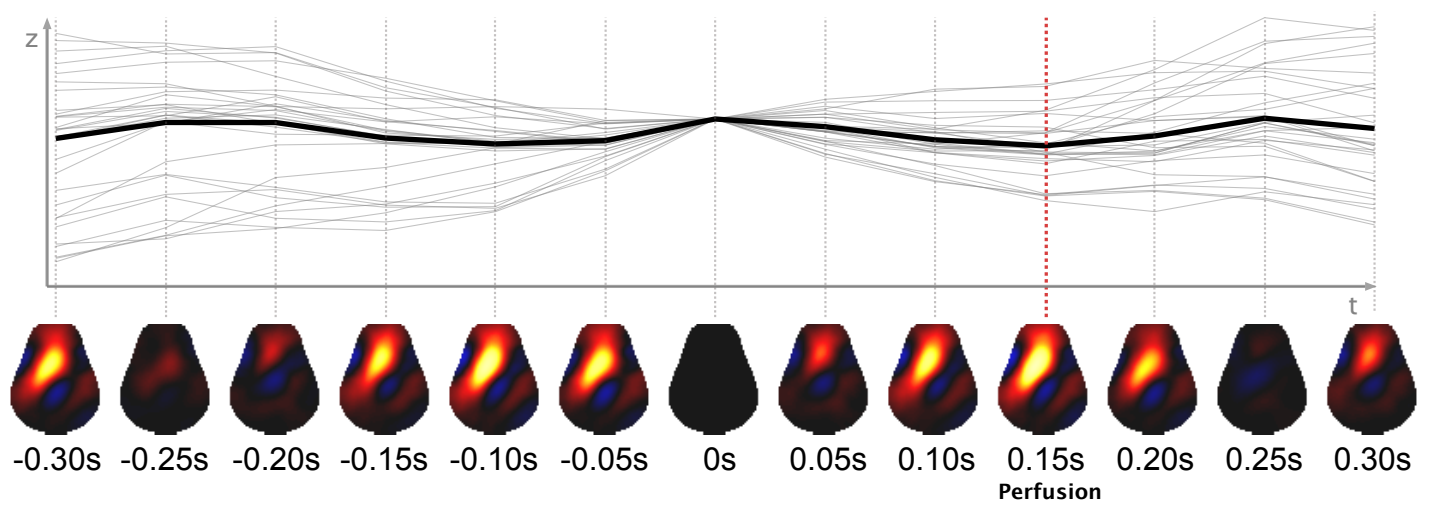

Figure 3.4: Illustration of the stages of the ensemble averaging process: A) an ensemble average of all heartbeats over the time frame is taken from the summed global signal; and B) shows reconstructed images corresponding to each time point in the global ensemble averaged signal above. The selected perfusion image is the image with the maximum impedance increase in the lung region.

\subsubsection{Image Comparison}

To compare the images the Jaccard score between functional EIT images was calculated. Negative impedance changes were removed from the images and the images were normalized.

The Jaccard score was calculated between the reference image calculated using the maximum increase in lung-region conductivity during bolus injection (b), and the frequency-based method (f): $J(x, y)=\sum_{i} \frac{\min \left(b_{i}, f_{i}\right)}{\max \left(b_{i}, f_{i}\right)}$ representing the distance between the two images. 


\subsubsection{Statistical Analysis}

To determine the significance of the change in bolus between postures and methods, the Cohen's d score was calculated to quantify the effect size of the change in the centre of mass of the perfusion image (Cohen, 1977). This was calculated as the difference between two means over the pooled standard deviation, where the difference between the two means is: $\mu_{1}-\mu_{2}$, and the pooled standard deviation is:

$$
\sqrt{\frac{\left(n_{1}-1\right) s_{1}^{2}+\left(n_{2}-1\right) n_{2} s_{1}^{2}}{n_{1}+n_{2}-2}} .
$$

\subsection{Results}

The Jaccard scores for each method were compared between ensemble averaging and frequency filtering methods to determine the regions where performance was best for each method. Figure 3.5 shows a comparison between Jaccard score for each animal, connecting lines indicate different methods performed on the same data segment, while each marker shape denotes a separate posture. 


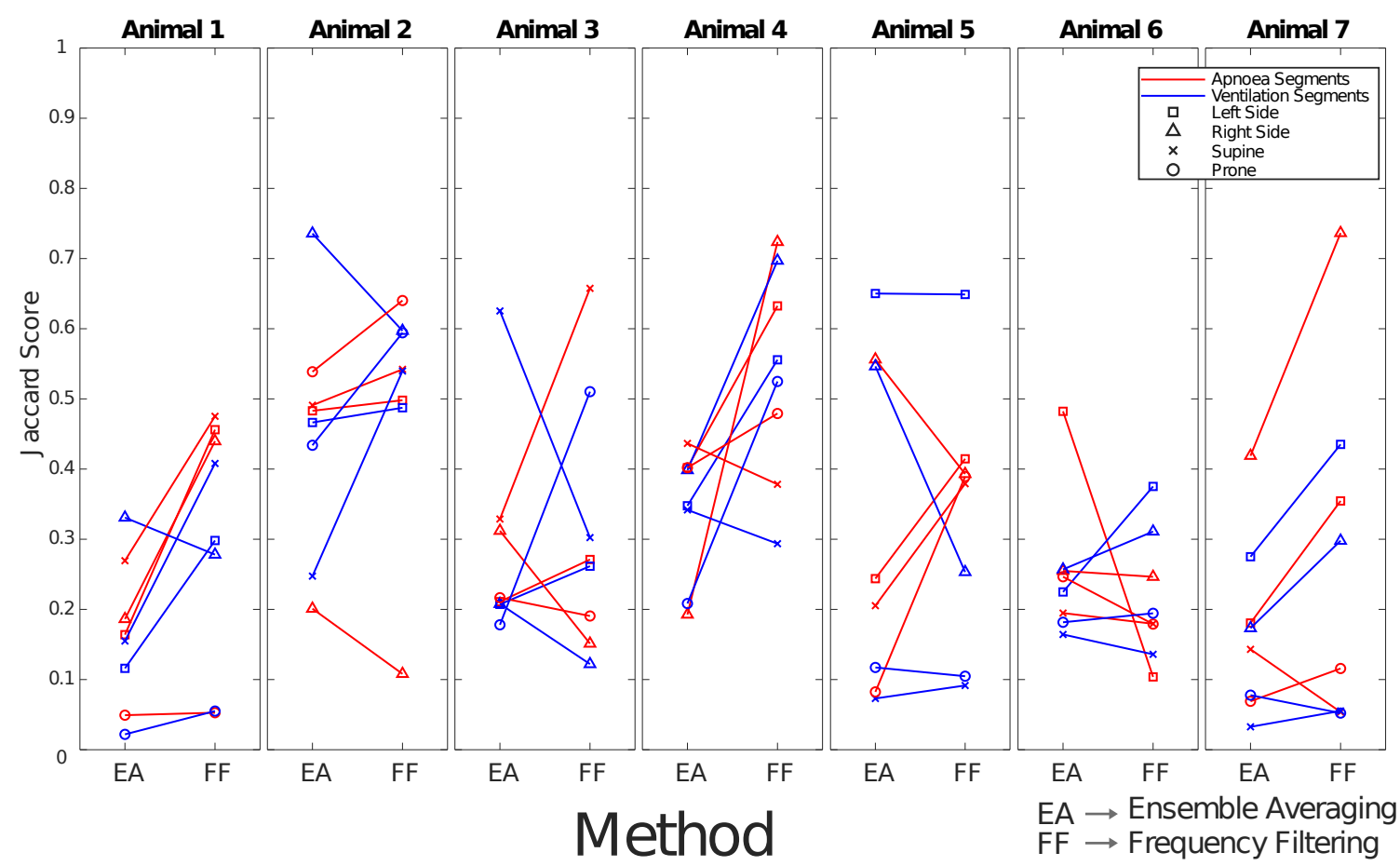

Figure 3.5: Jaccard scores for each method and animal in the comparison. Frequency filtering and ensemble averaging methods performed on the same data segment are connected by solid lines. Red lines and markers indicate apnoea data sections, while blue indicates ventilation data sections. Each posture is denoted by a different shaped marker in the figure.

On average frequency filtering outperforms ensemble averaging based methods of perfusion calculation $(\mathrm{p}=0.04)$, and there is no significant difference in performance of the heart-frequency based filtering techniques during periods of apnoea relative to periods of ventilation.

Of the 56 data regions that were analyzed, the ensemble averaging performed better in 12 cases and the frequency filtering achieved the best performance in 28 cases. There were 16 additional cases where the difference in performance was negligible at less than 5\%. On average, across all images, frequency filtering based methods 
scored $7 \%$ higher than ensemble averaging.

The centre of mass of the perfusion measure images using the bolus injection method had a Cohen's d score of less than 0.1 between posture changes indicating that there is an insignificant or trivial difference in the means relative to the standard deviation (Cohen, 1977). To demonstrate the visually observable changes due to posture change and the high similarities that can be observed between filteringand bolus-based perfusion estimates, frequency filtered images from animal 4 are compared to bolus based methods in figure 3.6 . 


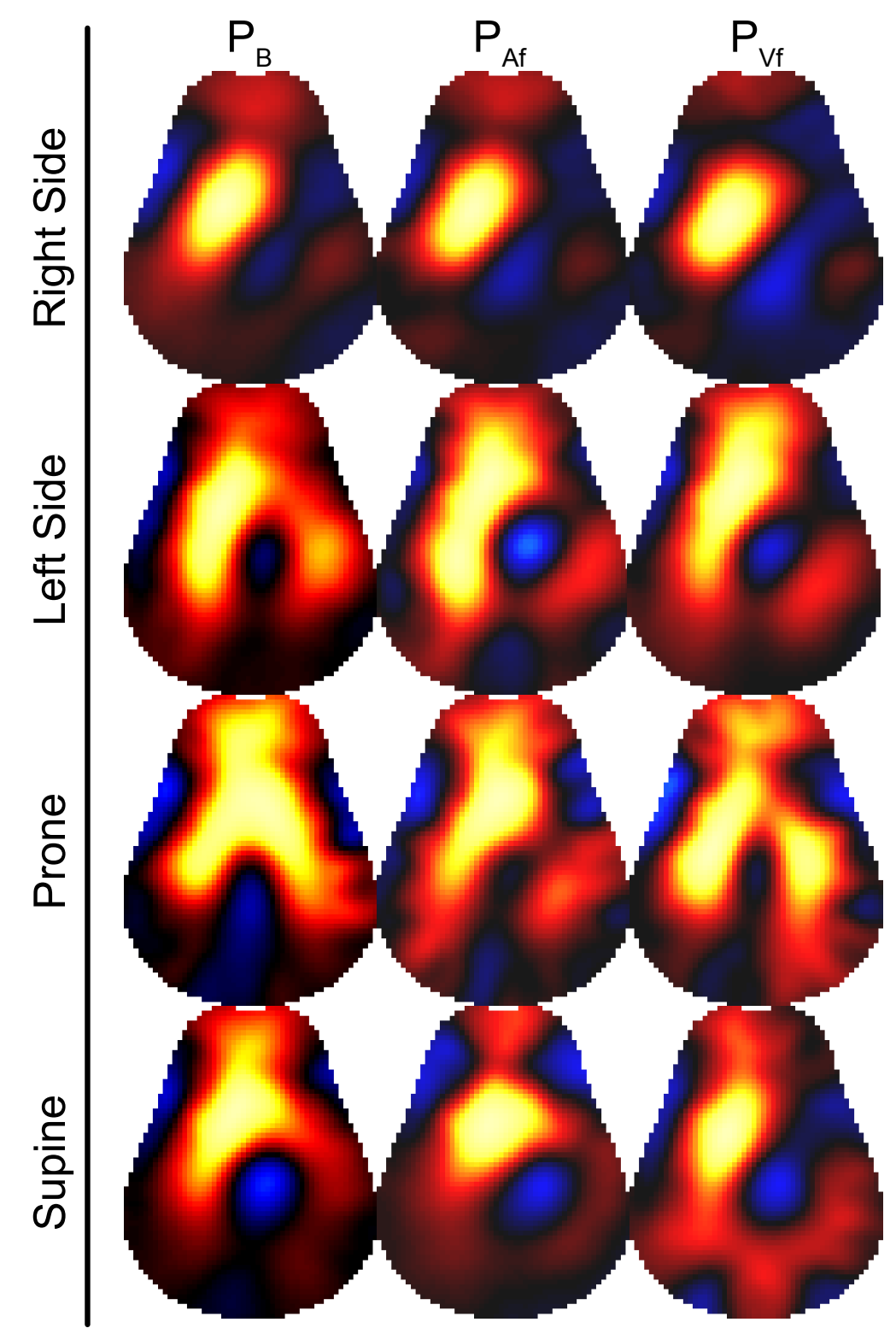

Figure 3.6: This figure shows the tracking of perfusion for frequency filtering measures of perfusion during apnoea and ventilation sections compared to bolus injection for animal 4 . $\mathrm{P}_{\mathrm{B}}$ is the bolus injection image, $\mathrm{P}_{\mathrm{Af}}$ uses the frequency filtering method during apnoea and $\mathrm{P}_{\mathrm{Vf}}$ is the frequency filtering method during ventilation. 


\subsection{Discussion}

Two primary approaches of EIT perfusion calculation have been compared in this paper: injection of a bolus of contrast agent resulting in EIT image changes which produce perfusion measures, and digital filtering of EIT image sequences to extract the heart-frequency components. Additionally, various algorithms have been evaluated for digital filtering-based approaches during mechanical ventilation and short apnoea sequences, using both frequency- and ensemble averaging-based techniques.

There have been few comparisons of these techniques, and we set out to better understand the relationship between perfusion and heart-frequency measures, and between the various filtering approaches used to determine heart-frequency cardiac changes. We selected an experimental protocol using posture change to alter the regional distribution of lung ventilation and perfusion in newborn lambs.

Our first question was "to what extent do heart-frequency filtering-based measures correspond to perfusion?"

The primary results (figure 3.5) use a Jaccard index of the similarity between functional images. Overall it was found that in healthy animals the Jaccard index indicated good agreement with our gold standard. While highly dependent on the data, it was found that there was a high degree of similarity between methods with respect to the overall shape of the perfusion. In both animals 2 and 4, where the signal required little preprocessing before analysis there is a higher Jaccard score across all postures.

The synchronization box was attached to the EIT system but was not used for this experiment, an error in the connection caused brief periods of the signal (less 
than $1 \mathrm{~s}$ ) in some animals to be zeroed. Through careful processing of this signal only brief sections of data were lost and we do not feel this impacts the results.

During the experiment the order of posture change was not randomized. While changes in ventilation due to posture change are not understood to have long-term physiological effects, if there is a longer-term effect of change in posture the lack of randomization will impact the results. Nguyen et al. (2015) were able to image perfusion changes due to induced pulmonary embolisms and using the peak impedance change on dilution curves. However our data presented insufficient variance in perfusion induced by posture change to complete a centre of mass analysis. A higher statistical power could potentially be achieved through initiating posture changes with more dramatic results in perfusion, such as upright to supine (Nakazato et al., 2010).

Throughout the experiment, the perfusion image was selected as the image containing the largest increase in conductivity in the sum of pixels in the lung region, which occurred at different relative times across animals and events. Many factors could affect this including belt positioning changes, and it could be a contributing factor to the inconsistent trends in amplitude changes in the global image across methods. In this ovine model, the belt placement was immediately behind the front legs. In this scenario there was potential for the belt to be in a slightly different position between animals due to size and positioning differences. In future studies or human recordings, it may be possible to place the belt more accurately using a more precise landmark such as the $4^{\text {th }}$ space.

Borges et al. (2012) compared EIT perfusion images using first-pass kinetics and 
heart-frequency filtering-based methods to perfusion measures using SPECT, finding that heart-frequency filtering techniques made systemic errors when used to estimate the perfusion. They also determined that there was no discernible relationship between the magnitude of the SPECT images and the heart-frequency images. This was consistent with the findings of this study that image amplitude of the bolus injection and heart-frequency filtering-based methods was not consistent in all animals. This methodology presented by Borges et al. (2012) was not part of the comparison in this study as the perfusion signal due to the heart could not be consistently identified and removed across all animals. In two dimensions, heart-frequency and ventilation signals have been used to identify the location of the heart and lungs within the EIT electrode plane with known electrode locations and anatomy (Ferrario et al., 2012), but in situations where the electrode location and anatomy are not precisely known EIT tends to perform poorly as a structural imaging modality (Adler and Boyle, 2017). These challenges suggest that configurations with multiple planes of electrodes may be better able to isolate and remove off-plane pulsatility signals related to the heart.

It was observed that the general shape of the perfusion was consistent across all methods despite amplitude variations. One reason for the difference in amplitude change across animals may be due to slight variations in the belt placement and electrode positioning on the animals. If the belt is closer to the heart, there will be a larger heart-frequency component to the signal and there may be a variance in the impedance change due to bolus injection. This study measures perfusion using filtering techniques during apnoea while the bolus injection takes place. The filtering 
approaches are used to eliminate the low frequency change due to the injection of the saline bolus. While the conductivity of blood may be increased after the bolus injection, we do not expect that this will influence the filtering approaches that isolate the cardiac-frequency activity.

Next, we asked, "what are the advantages and disadvantages of different approaches to heart-frequency filtering of EIT data, and which techniques are recommended under which circumstances?"

Our overall recommendation is that, whenever possible, frequency filtering techniques should be used. This is largely because frequency filtering methods tend to be more stable in the presence of noise on the signal. Ensemble techniques are advantageous in some circumstances, because they better use the heart-frequency variability to avoid interference from harmonics of the ventilation at the heart rate. For frequency-filtering techniques, it is necessary to widen the heart-frequency filters to account for such variability. On the other hand, it is sometimes not possible to accurately synchronize heartbeats, due to noise corruption in the signals or the very low amplitude of the heart-frequency signals relative to the ventilation signal. In cases where the signal of the heartbeat was not clearly identifiable through visual inspection of the signal, neither ensemble averaging nor frequency filtering was able to achieve good estimates of perfusion relative to the bolus injection event.

In summary, our goal was to understand the relationship between bolus- and filtering-based EIT measurements of lung perfusion, as well as the relationship between different filtering-based measures of perfusion. Our results indicate there is a common trend between the shape and perfusion estimates of both heart-frequency 
and bolus injection images despite the difference in physiological events behind each measure. Amongst filtering techniques, frequency filtering outperforms ensemble averaging across regions of data where there is noise present and the heart signal cannot be readily identified, and both methods were able to approximate the bolus injection measures equally well when applied to apnoea and ventilation regions of data.

\subsection{Summary}

Two main functional imaging approaches have been used to measure regional lung perfusion using Electrical Impedance Tomography (EIT): venous injection of a hypertonic saline contrast agent and imaging of its passage through the heart and

lungs, and digital filtering of heart-frequency impedance changes over sequences of EIT images. This paper systematically compares filtering-based perfusion estimates and bolus injection methods to determine to which degree they are related. EIT data was recorded on 7 mechanically ventilated newborn lambs in which ventilation distribution was varied through changes in posture between prone, supine, left- and right-lateral positions. Perfusion images were calculated using frequency filtering and ensemble averaging during both ventilation and apnoea time segments for each posture to compare against contrast agent-based methods using the Jaccard score. Using bolus-based EIT measures of lung perfusion as the reference frequency filtering techniques performed better than ensemble averaging and both techniques were applied successfully across both apnoea and ventilation data segments. Our results indicate the potential for use of filtering-based EIT measures of heart-frequency ac- 
tivity as a non-invasive proxy for contrast agent injection-based measures of lung perfusion. 


\section{Chapter 4}

\section{FEM Mesh Refinement for 3D EIT}

\subsection{Motivation}

This thesis proposes the use of novel 3D electrode configurations and internal electrodes to improve sensitivity to cardiosynchronous activity. Unlike surface electrode configurations, models with internal electrodes have a very high sensitivity in the centre of a model where mesh refinement can be challenging. In this chapter we explore the need for mesh refinement with regard to sensitivity accuracy, and develop tools to help control mesh density.

\subsection{Introduction}

EIT is being actively developed to image the movement of air and blood in the thorax (Nguyen et al., 2012), and neurological activity (Holder, 1992). For most applications of EIT the finite element method (FEM) is used to reconstruct images, 
since geometry is irregular and an analytic solution cannot be used. When using the FEM, a mesh is required to reconstruct images. To reconstruct images we require the solution to an inverse problem and therefore we must calculate the sensitivity or jacobian. As described in section 2.4.5, a jacobian matrix represents relationship between the voltage measurements and internal conductivity. In this thesis we use the term mesh to refer to a specific combination of nodes and elements in a finite element model. As more elements are added to a mesh, the accuracy is improved, but the time required to reconstruct and display results is increased. In order to generate accurate results, a mesh that gives the highest accuracy with the fewest nodes is desirable. This way accuracy can be increased, and computing time can be decreased.

Due to the propagation of current in EIT, sensitivity is much higher near the electrodes relative to the centre of the model. This results in an inverse problem that is severely ill-posed, and an uneven sensitivity distribution in the model. An accurate calculation of the jacobian is required to solve the inverse problem accurately, and errors in regions with high sensitivity will disproportionately affect the sensitivity error and the resulting reconstructed image.

It has generally been recommended in EIT literature that meshes be refined near electrodes, where the electric field and sensitivity are largest (Adler and Boyle, 2017) but no thorough analysis has been made to determine how much refinement is required. This recommendation gives rise to two questions: 1) Given a "mesh element budget," what should balance of nodes be between the centre of the model and the electrodes? And 2) How do different freely available meshing tools that are 
commonly used with EIT compare when used to refine 3D meshes?

Previously with EIT, mesh refinement has primarily been either constant, or based on the complexity of geometric surfaces and lines within a model (Grychtol and Adler, 2013). In EIDORS (Adler and Lionheart, 2006) meshes are generated using both Netgen (Schöberl, 1997) and Gmsh (Geuzaine and Remacle, 2009) for 2D and 3D models. Refinement around electrodes is commonly performed by setting a mesh density for the electrodes and allowing the mesh density to decay towards the maximum mesh size. This does not allow the user to specify the rate of decay or precisely control the mesh size.

A model that accurately represents the anatomy of the imaged region can greatly increase the quality of the reconstructed image (Grychtol et al., 2012), but increasing the complexity of mesh surfaces presents additional challenges for mesh refinement. EIT reconstruction software EIDORS enables users to place electrodes on the surface of complex boundaries (Grychtol and Adler, 2013), but the current functionality does not enable control of the refinement around the electrodes or internal structures. Most commercially available FEM packages do not conveniently provide such capability either.

In this chapter we investigate approaches to manage the tradeoff between refinement of the electrode regions versus the bulk volume. We present a comparison between Gmsh and Netgen based mesh refinement around electrodes, and evaluate the effect of mesh refinement techniques on error in the sensitivity matrix, $\mathbf{J}$. 


\subsection{Methods}

\subsubsection{Overview}

We built a cylindrical model in Gmsh and Netgen which was parameterized so that multiple different combinations of mesh refinement were possible. These results were compared to a very high density meshes which was considered the gold standard. The format of the geometry files used to generate these meshes can be seen in appendix A

\subsubsection{Mesh Generation}

A cylinder (with a diameter of $0.5 \mathrm{~m}$, and a height of $0.25 \mathrm{~m} \mathrm{~h}=0.25 \mathrm{~m}$ ) with four square electrodes ( $5 \mathrm{~cm}$ edge length) placed equidistantly around the perimeter at mid-height was meshed with Netgen (version 5.3.1) (Schöberl, 1997) and Gmsh (version 4.7.0) (Geuzaine and Remacle, 2009) meshing software. Current was injected between adjacent electrodes and the voltage was measured between the remaining two electrodes. For 3D meshes an initial analysis was done building on work from Grychtol and Adler (2013) where mesh density was set by specifying the maximum edge lengths permitted on electrode surfaces and in the volume of the FEM. Results were compared against those generated using ultra-fine meshes. Calculations were performed with EIDORS (version 3.10) (Adler and Lionheart, 2006) in Matlab 2019b (The Mathworks, Natick, MA, USA).

Meshes of different sizes were generated with Netgen and Gmsh by manipulating the desired maximum edge length (maxh parameter) for the entire domain and the electrodes. Two mesh analyses were performed. For the first mesh, maximum element 
edge lengths were chosen such as to divide the electrode side of $5 \mathrm{~cm}$ into an integer number of segments of equal size. The maximum mesh edge length ranged from 1 to 7 subdivisions of the electrode edge, while the maximum mesh edge length in the ultrafine reference mesh was 15 subdivisions per electrode edge. Independent reference meshes were generated for each software. Two types of models were generated this way. Constant models $\mathrm{C} 1-\mathrm{C} 7$, where the mesh size was constant, and refined models R1-R7 where the electrode mesh size was specified and dissipated towards an internal mesh element size of $5 \mathrm{~cm}$. Additional refined meshes were generated with reduced mesh size in the internal mesh regions. R1-R7 is referred to as refinement A where the internal element max size was $5 \mathrm{~cm}$. Refinement B had an internal mesh size of $4 \mathrm{~cm}, \mathrm{C}$ was $3 \mathrm{~cm}$ and D was $2 \mathrm{~cm}$. The numeric value in the mesh ID indicated the number of subdivisions per electrode edge. In Netgen the mesh decay was not easily controlled by selecting a refinement level from 1 to 5 , but in Gmsh the size was set to increase evenly from the surface of the electrode to the centre of the model. It should be possible to replicate this mesh density distribution Netgen by using a separate mesh density file that specifies the required density at each point in the geometry, but this technique is not widely used due to its complexity. Figure 4.1 shows example meshes of coarse, fine and refined meshes. Figure 4.2 shows the generated mesh structure for constant refinement meshes around the electrode for both Netgen and Gmsh. 


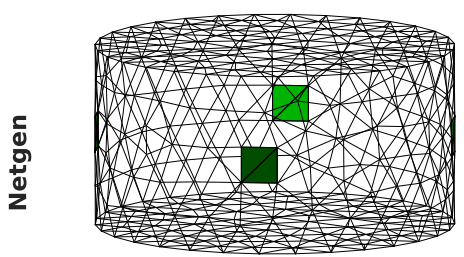

(a) $\mathrm{C} 1$

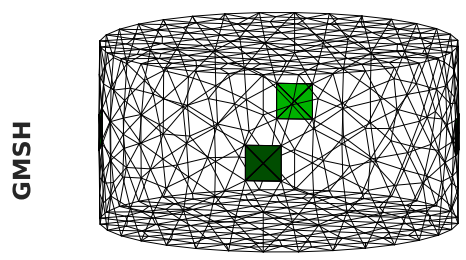

(a) $\mathrm{C} 1$

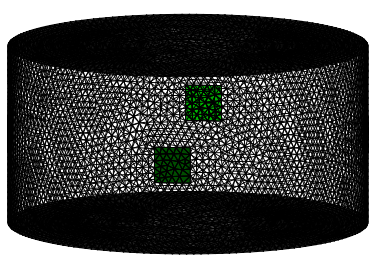

(b) $\mathrm{C} 5$

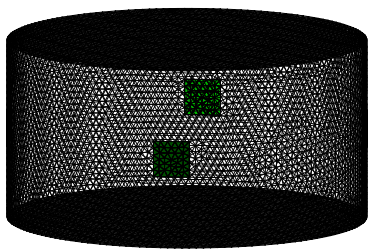

(b) $\mathrm{C} 5$

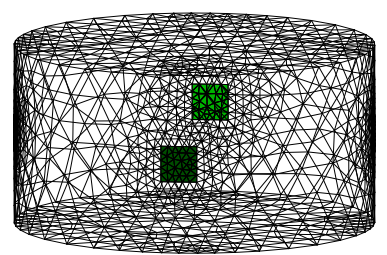

(c) R5

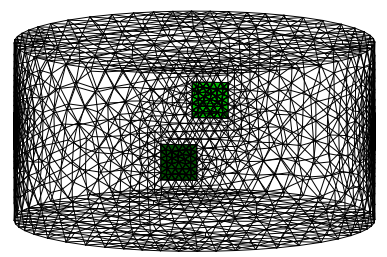

(c) R5

Figure 4.1: Sample meshes generated with Netgen (top row) and Gmsh (bottom row). From left to right: (C1) the coarsest constant mesh; (C5) a refined constant mesh; and (R5) a refined mesh with the same electrode mesh density as C5 but lower internal mesh density. 

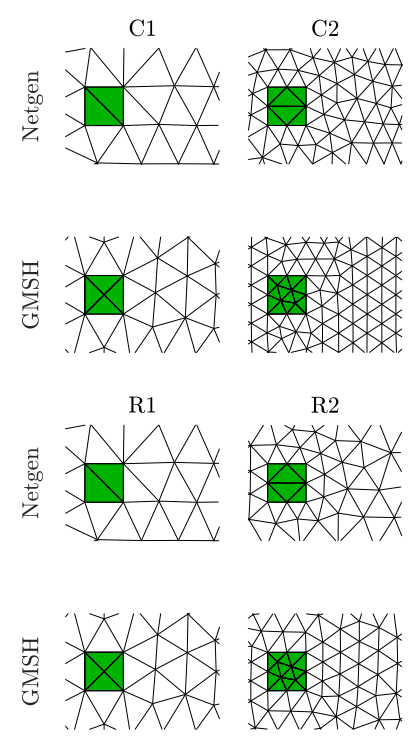

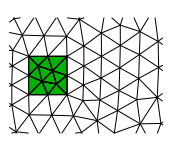

$\mathrm{C} 2$
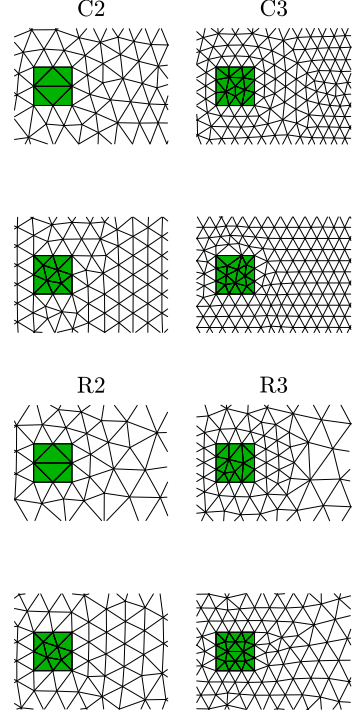
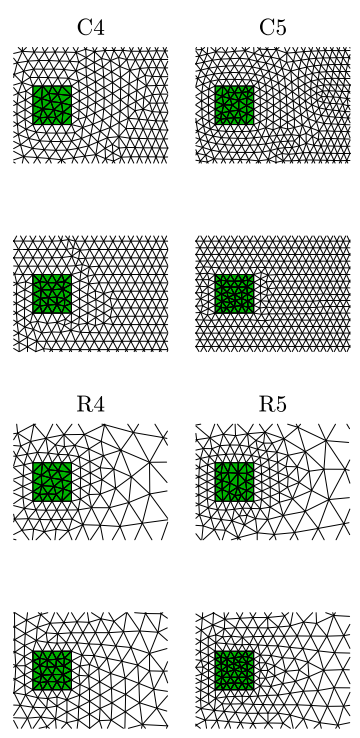
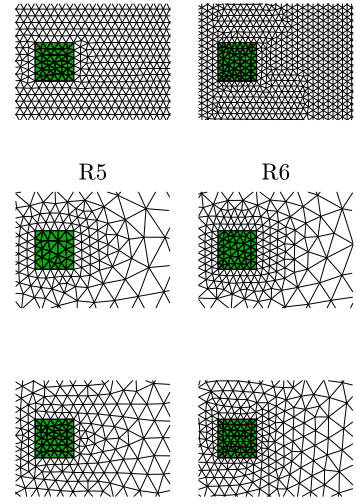

C6
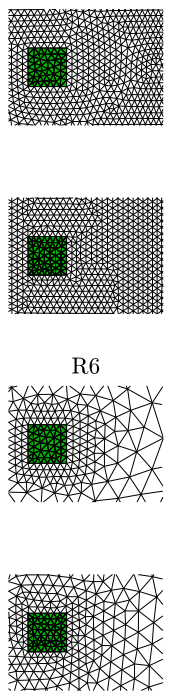
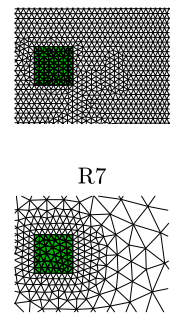

$\mathrm{C} 7$
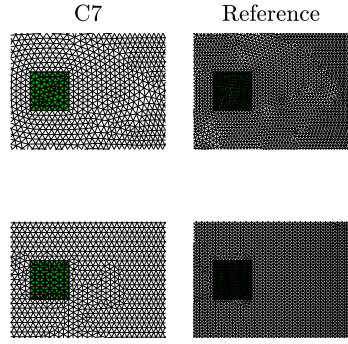

Reference
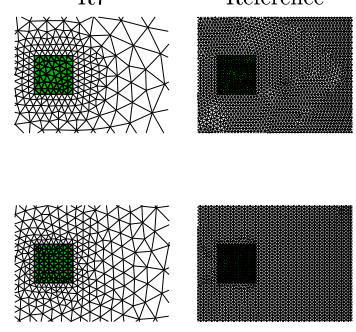

Figure 4.2: A view of the electrode meshing for all constant-density meshes in Netgen and Gmsh. The top two rows show all electrode faces and immediate surrounding surroundings from coarsest $(\mathrm{C} 1)$ to finest $(\mathrm{C} 7)$. C represents the constant mesh refinement and the number represents the specified mesh subdivisions per electrode edge. The reference mesh is equivalent to $\mathrm{C} 15$. The bottom two rows show refined meshes R1 to R7 with both Netgen and Gmsh and shows the rate of mesh dissipation away from the electrodes.

For the second analysis, the distribution of nodes within the model was changed without altering the total number of nodes to give M1-M17. Starting with the constant mesh C3 as M1, the maximum mesh element length on the electrode was decreased by $10 \%$ and the maximum mesh size in the centre was increased so that the total number of elements in the mesh was within $10 \%$ of the original mesh. C3 was chosen as the starting point because several steps of mesh refinement could be generated before the electrode mesh density surpassed the reference meshes. For mesh M17 the specified electrode refinement was equal to the reference mesh. In Netgen the mesh dissipation rate was not further controlled, and in Gmsh the mesh 
density decreased evenly from the electrode surface to the centre of the model. To compare these meshes a section of the model was selected encompassing all points between the centre of the model and a selected electrode face. The average distance, or balance point, along the x-axis of the selected points was expressed as a percentage of the tank radius. This process is illustrated in figure 4.3 .
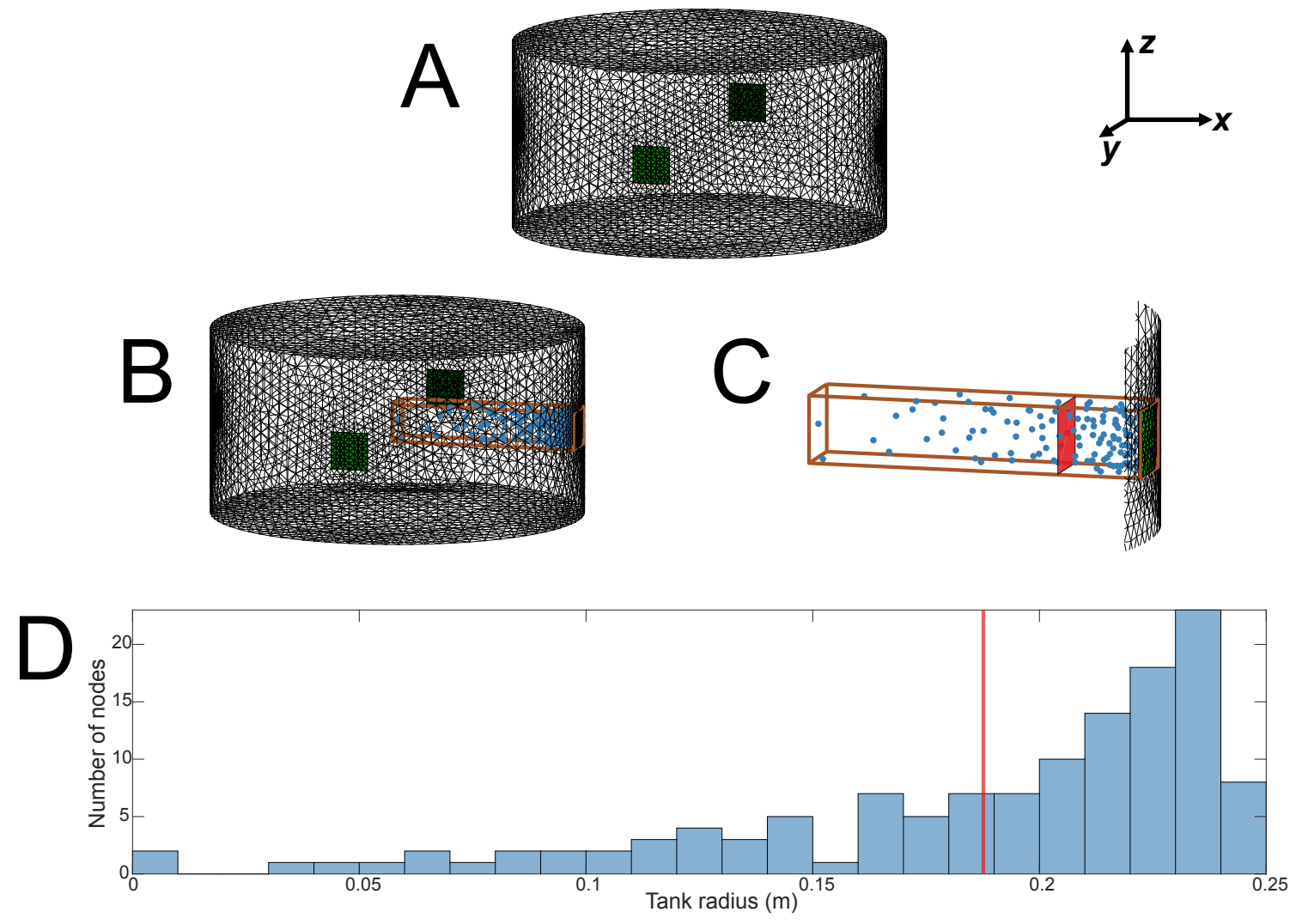

Figure 4.3: A sketch of the process to determine the balance point of generated meshes. A) the starting mesh; B) nodes between the electrode surface and the centre of the model are identified; C) the balance point of the nodes along the $\mathrm{x}$-axis is calculated and indicated by the red plane; D) a histogram showing an example distribution and balance point (red) for the selected model.

When generating meshes to compare across several mesh density profiles as the 
Table 4.1: Mesh parameters for odd numbered meshes generated by Netgen (A) and Gmsh (B) to determine the optimal node balance. Parameters global maxh and electrode maxh refer to the specified input parameters; the remaining columns give parameters from the resulting meshes.

\begin{tabular}{cccc|ccc|llll} 
Mesh ID & $\begin{array}{l}\text { glbl. } \\
\text { maxh }\end{array}$ & $\begin{array}{l}\text { elec. } \\
\text { maxh }\end{array}$ & \# elem. & \# nodes & $\begin{array}{l}\text { \# elec. } \\
\text { elem. }\end{array}$ & $\begin{array}{l}\text { minEL } \\
{[\mathrm{mm}]}\end{array}$ & $\begin{array}{l}\text { maxEL } \\
{[\mathrm{mm}]}\end{array}$ & $\begin{array}{l}\text { minEV } \\
{\left[\mathrm{mm}^{3}\right]}\end{array}$ & $\begin{array}{l}\text { maxEV }^{d} \\
{\left[\mathrm{~mm}^{3}\right]}\end{array}$ \\
\hline \multirow{2}{*}{ M-01 } & A & 16.67 & 16.67 & 31347 & 7095 & 22 & 9.80 & 49.45 & 254.76 & 6851.01 \\
& B & 16.67 & 16.67 & 49210 & 9615 & 25 & 10.32 & 50.00 & 222.87 & 2898.59 \\
M-03 & A & 18.33 & 15.00 & 29639 & 6482 & 22 & 10.75 & 50.41 & 289.80 & 5826.14 \\
& B & 18.33 & 15.00 & 49247 & 9680 & 40 & 7.36 & 37.11 & 172.78 & 2814.55 \\
M-05 A & 18.33 & 13.33 & 29814 & 6589 & 28 & 9.39 & 49.91 & 162.26 & 5648.41 \\
& B & 20.00 & 13.33 & 50749 & 9930 & 42 & 7.80 & 37.93 & 134.41 & 3233.59 \\
M-07 A & 18.33 & 11.67 & 30581 & 6723 & 36 & 8.77 & 47.88 & 141.74 & 6252.36 \\
& B & 21.67 & 11.67 & 53002 & 10429 & 60 & 6.17 & 40.84 & 63.22 & 4077.18 \\
M-09 A & 18.33 & 10.00 & 30690 & 6755 & 42 & 7.86 & 49.18 & 115.45 & 5496.39 \\
& B & 23.33 & 10.00 & 56237 & 11008 & 68 & 5.82 & 43.81 & 62.88 & 4962.89 \\
M-11 A & 18.33 & 8.33 & 31575 & 7030 & 74 & 6.05 & 50.99 & 60.06 & 6086.88 \\
& B & 26.67 & 8.33 & 55545 & 10886 & 96 & 5.51 & 49.72 & 36.84 & 7424.70 \\
M-13 A & 20.00 & 6.67 & 28589 & 6447 & 92 & 4.65 & 51.85 & 20.68 & 6664.11 \\
& B & 30.83 & 6.67 & 54993 & 10825 & 148 & 4.51 & 55.36 & 20.63 & 10453.90 \\
M-15 A & 21.67 & 5.00 & 27775 & 6245 & 158 & 3.46 & 52.60 & 11.13 & 9097.30 \\
& B & 36.67 & 5.00 & 55331 & 11000 & 250 & 3.51 & 61.66 & 7.99 & 15838.09 \\
M-17 A & 30.00 & 3.33 & 39116 & 7590 & 320 & 1.75 & 72.83 & 1.13 & 23783.17 \\
& B & 48.33 & 3.33 & 52947 & 10798 & 548 & 2.32 & 86.60 & 2.72 & 32287.34 \\
\hline \multirow{2}{*}{ REF } & A & 3.33 & 3.33 & 6661789 & 1173243 & 510 & 1.75 & 9.49 & 1.01 & 46.09 \\
& B & 3.33 & 3.33 & 5871464 & 976558 & 554 & 2.10 & 7.59 & 1.58 & 21.65 \\
& & $a:$ & minimum mesh edge length, $b:$ maximum mesh edge length & \\
& & $c:$ & minimum mesh element volume, & $d:$ maximum mesh element volume &
\end{tabular}

balance of the nodes was shifted towards the electrodes, 19 meshes were generated for each software including 2 reference meshes. Table 4.1 shows the parameters of the resulting odd numbered meshes. 


\subsubsection{Simulation}

To calculate the sensitivity of each measurement $\left(\partial v_{j}\right)$ to a change in conductivity $\left(\partial \sigma_{i}\right)$ of each element we use the adjoint method (Polydorides and McCann, 2002) where the sensitivity is given by the jacoabian $(J B)$.

$$
\mathbf{J}_{i j}=\frac{\partial v_{j}}{\partial \sigma_{i}}
$$

The sensitivity was normalized by dividing the sensitivity value for each element by the element's volume. The average sensitivity of 15 planes evenly spaced between the top and bottom edges of the electrodes was used to generate a sensitivity image. The EIT image structure was rasterized onto a $512 \times 512$ array, and divided into 3 regions of interest: the centre of the model (C), adjacent to the electrode (E) and between the centre and electrode (I). The resulting sensitivity for the reference mesh calculated with Gmsh and the selected regions of interest are presented in figure 4.5 .

To calculate the sensitivity error, the difference in sensitivity was computed between the reference model and the refined models to give the total error in sensitivity. The normalized sensitivity error is calculated as the absolute sensitivity error divided by the total sensitivity error for the selected region.

\subsubsection{Electrode Refinement for Arbitrary FEMs}

Our approach for refinement around electrodes in Gmsh with external electrodes also allows for the refinement of arbitrary models with complex structures such as internal electrodes and tissue boundaries. A scenario depicting an approximation 
of a probe entering a bone with different layers of conductivity was modelled. The resulting mesh pictured in figure 4.7 highlights the ability of this technique to be used for refinement around electrodes and the control of mesh density surrounding internal structures which was previously very challenging in EIT software.

\subsection{Results}

Two analyses of mesh refinement were completed. First, comparing normalized sensitivity error between meshes with constant refinement and refinement only at the electrodes; Second, comparing meshes with different levels of electrode refinement and the same number of nodes.

When comparing constant meshes to meshes with refinement at the electrodes only, normalized sensitivity error was decreased as more nodes were added to the mesh and to the electrodes. The sensitivity error was lowest in the constant meshes across both Netgen and Gmsh software. Meshes generated using Netgen provided a slightly lower sensitivity error relative to the respective reference mesh compared to Gmsh, and resulted in meshes with fewer nodes per electrode given the same input parameters. figure 4.4 shows the sensitivity error between constant and refined meshes with respect to the number of nodes per electrode. 


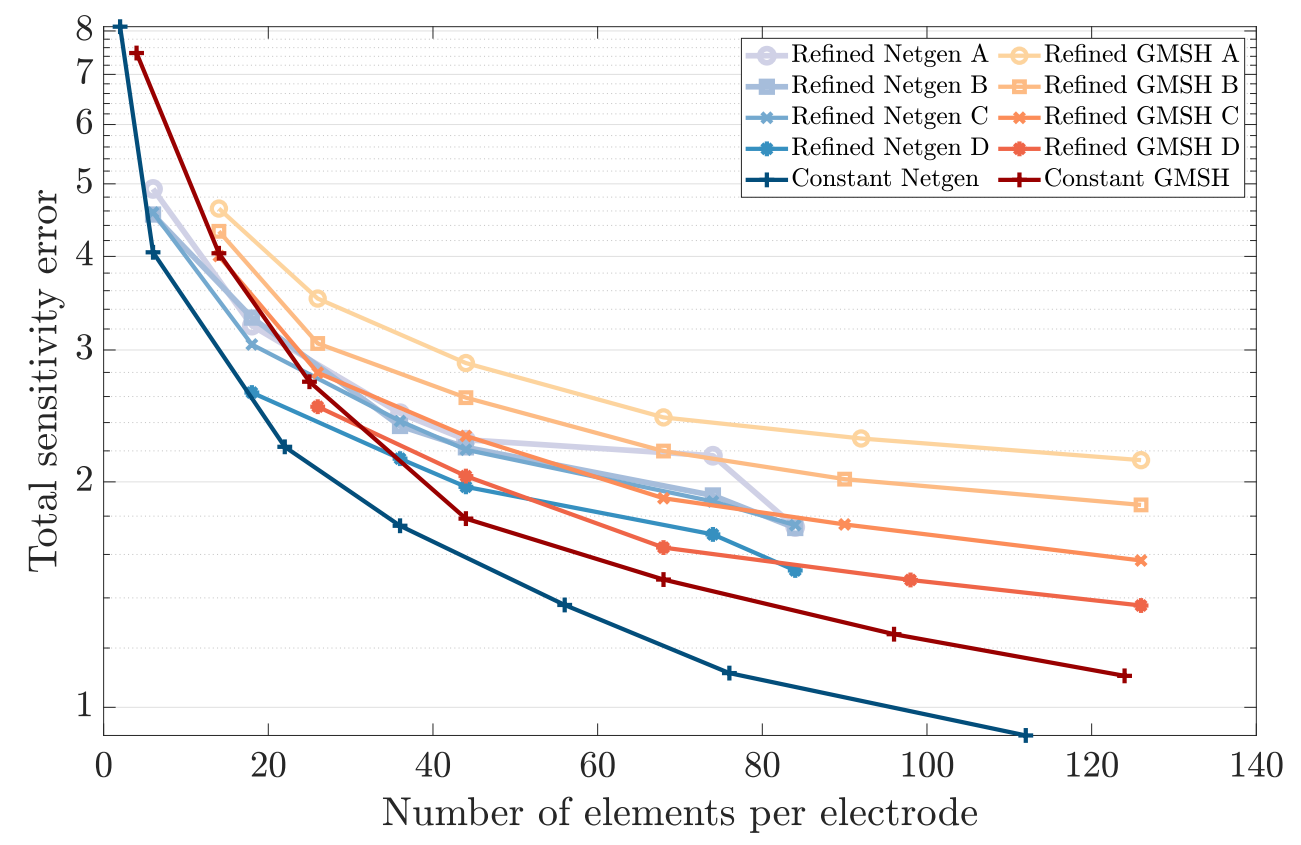

Figure 4.4: Normalized sensitivity error of each mesh as a function of the number of elements per electrode for both Netgen and Gmsh. The darkest lines indicate the constant mesh refinement, lighter lines indicate a larger maximum internal mesh size. The maximum internal mesh sizes are as follows: refinement A $-5 \mathrm{~cm}$; refinement B $-4 \mathrm{~cm}$; refinement C - $3 \mathrm{~cm}$; refinement D - $2 \mathrm{~cm}$.

Example sensitivity profiles for the M-series meshes are shown in figure 4.5. The resulting sensitivity profile near the electrodes more closely matched the reference case when refinement at the electrodes was higher. 
(A)

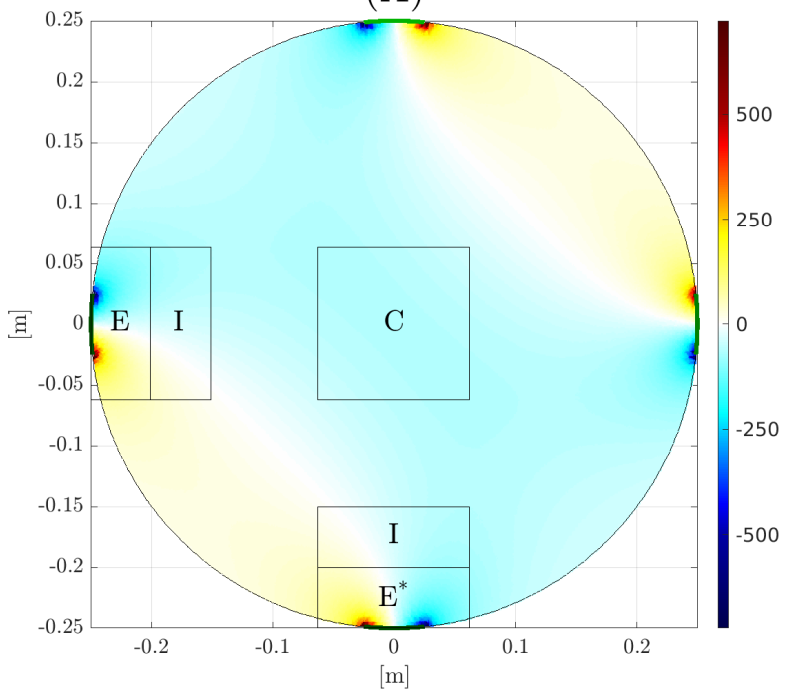

(B)

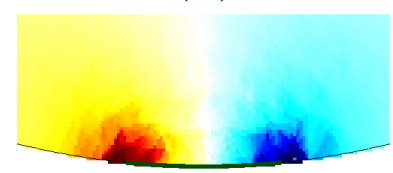

(i)

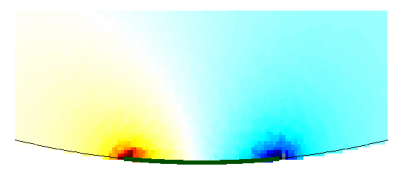

(ii)

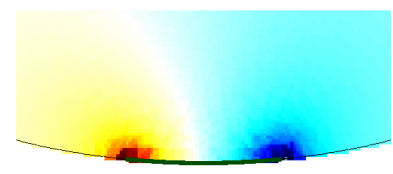

(iii)

Figure 4.5: (A) Sensitivity distribution for the reference mesh (C15) generated in Gmsh with regions of interest used to compare between models. (B) 3 sensitivity distributions in region $\mathrm{E}^{*}$ next to the electrodes: (i) Constant mesh M1 (ii) refined mesh M15 with a balance point of $82 \%$ (iii) reference mesh from (A).

The total sensitivity error across all meshes is plotted vs. the balance point in figure 4.6. For meshes generated with Gmsh the minimum error was achieved when the node balance point was approximately $85 \%$ of the model radius corresponding to model M15-B. For Netgen generated meshes, the minimum sensitivity was achieved in model M13-A at a balance point of approximately $70 \%$. Gmsh achieved a lower sensitivity error measured against the respective reference mesh. For meshes using Netgen refinement, the balance point did not increase evenly as the electrode density was increased and the internal density decreased. To maintain the same number of nodes within the model, Gmsh required a larger internal maxh than Netgen. Gmsh generated meshes with more nodes for the same input parameters, but generally the resulting mesh sizes were closer to those specified. The resulting mesh parameters for 
odd numbered meshes can be seen in table 4.1. Across all meshes the measurement error when computing the voltage measurements was insignificant at less than $0.2 \%$. 

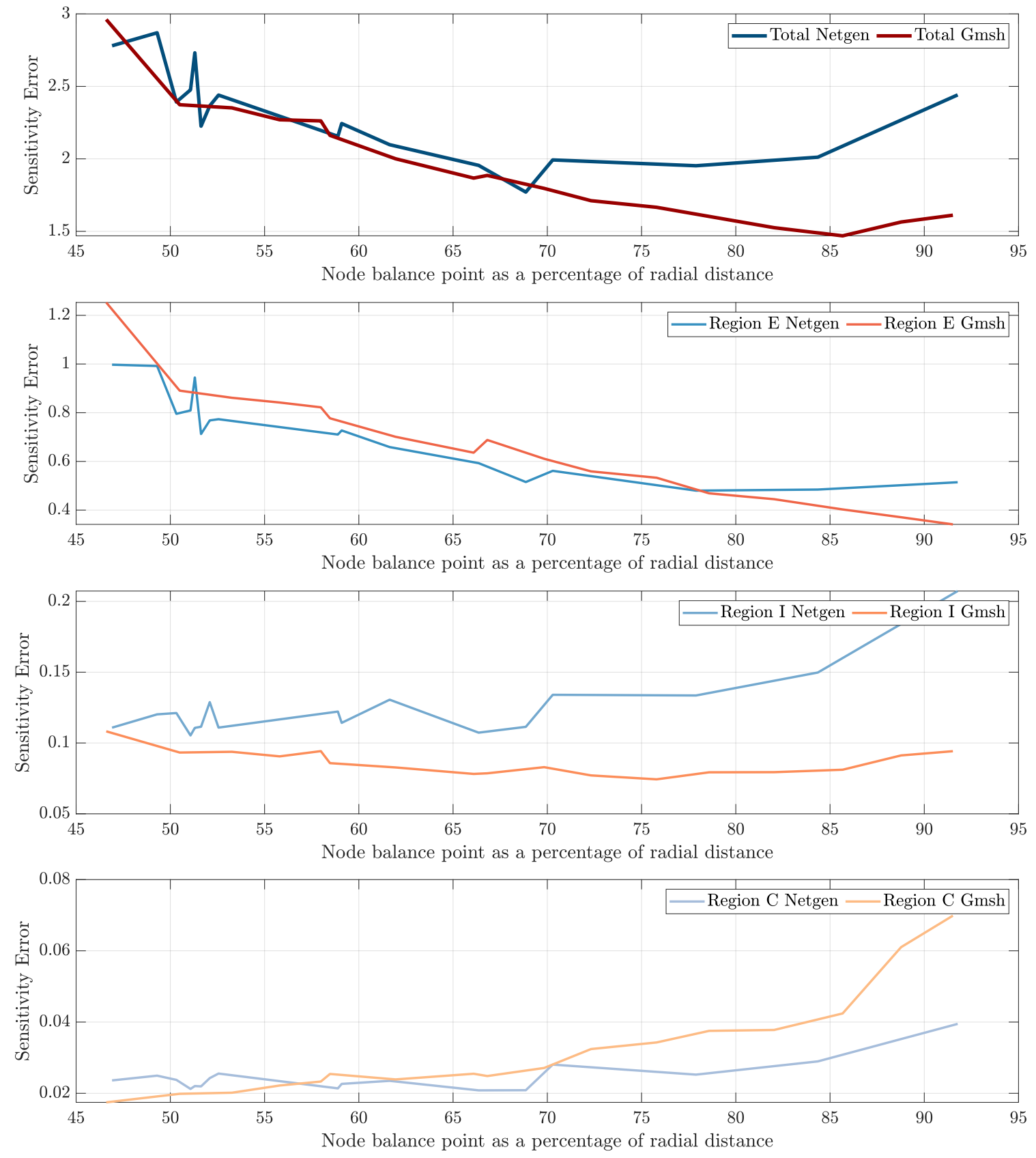

Figure 4.6: Resulting sensitivity error for Netgen (blue) and Gmsh (red) as the balance of the nodes was shifted towards the electrodes. From top to bottom: total sensitivity error, the sum of sensitivity error in region E, the sum of sensitivity error in region I and the sum of sensitivity error in region C. The regions are described in figure 4.5 . 


\subsection{Discussion}

We consider several questions on the requirement of FEM refinement near electrodes and the available tools for mesh refinement in EIT. 1) Given a "FEM element budget," what should balance of nodes be between the centre of the model and the electrodes? 2) How do different freely available meshing tools that are commonly used with EIT compare when used to refine 3D meshes?

While refining meshes surrounding the electrodes is agreed to be useful, there is a lack of systematic analysis of the required refinement level, and controlling such refinement is difficult. Automatic mesh refinement is an area of active work and there are a number of commercial and free products available. We compare two programs used widely in EIT. Our results show that Netgen and Gmsh control mesh refinement differently and the same input parameters result in meshes with different numbers of nodes. figure 4.2 depicts the difference in mesh dissipation rates between Netgen and Gmsh. The mesh size in Gmsh increased gradually from the surface of the electrode towards the centre of the model, whereas the mesh size in Netgen increased much more quickly from the edges of the electrode. While we attempted to control the dissipation rate in Netgen by manipulating the mesh density in the centre of the model, we were unable to achieve a smooth transition between the electrode and internal regions of the mesh.

To analyze the benefit of electrode refinement and the difference between Netgen and Gmsh refinement techniques we consider a sequence of refined meshes compared to a "gold standard," uniformly fine FEM solution. The models were refined either globally or from the electrode surface, and the error in the sensitivity matrix $\mathbf{J}$ 
was compared. figure 4.4 displays the difference in sensitivity between constant meshes and meshes with refinement at the electrodes. The sensitivity error was lower in Netgen across all constant refinement meshes, in part because there were more total nodes than Gmsh meshes with the same number of nodes on the electrode. Refinement around the electrode decreased the total sensitivity error, but still had larger error than meshes with constant refinement due to the smaller number of nodes in the model.

Using the balance point analysis, we were able to determine the optimal distribution of nodes to minimize sensitivity in both Gmsh and Netgen. The minimum total sensitivity error was achieved with a balance point of approximately $70 \%$ for Netgen. This can be seen in figure 4.6. This was mainly due to the rapid dissipation of node density away from the electrodes. Increasing the refinement near the electrode reduced error in region $\mathrm{E}$, but in region I there were insufficient nodes to reduce the sensitivity error. As the balance of refinement approached $90 \%$ the error in region E also started to increase as the node density did not remain fine throughout the entire region. This effect can also be seen in figure 4.2. In Gmsh the optimal balance for refinement was at approximately $85 \%$ of the radial distance. Since mesh density was set to reduce evenly between the electrode surface and the centre of the model, there was a higher density of nodes that was maintained in regions E and I as the electrode refinement increased. The error in the centre of the model was higher in Gmsh meshes, but since this is where the sensitivity is lowest, the total sensitivity error remained lower.

The ability to selectively control mesh refinement in Gmsh also allows users to 
generate complex meshes and control mesh density surrounding internal structures and electrodes. An example mesh is shown in figure 4.7 that depicts a probe entering a bone from the surrounding tissue. These additions fill an important need in EIT to allow for more accurate models of regions surrounding internal structures and electrodes.
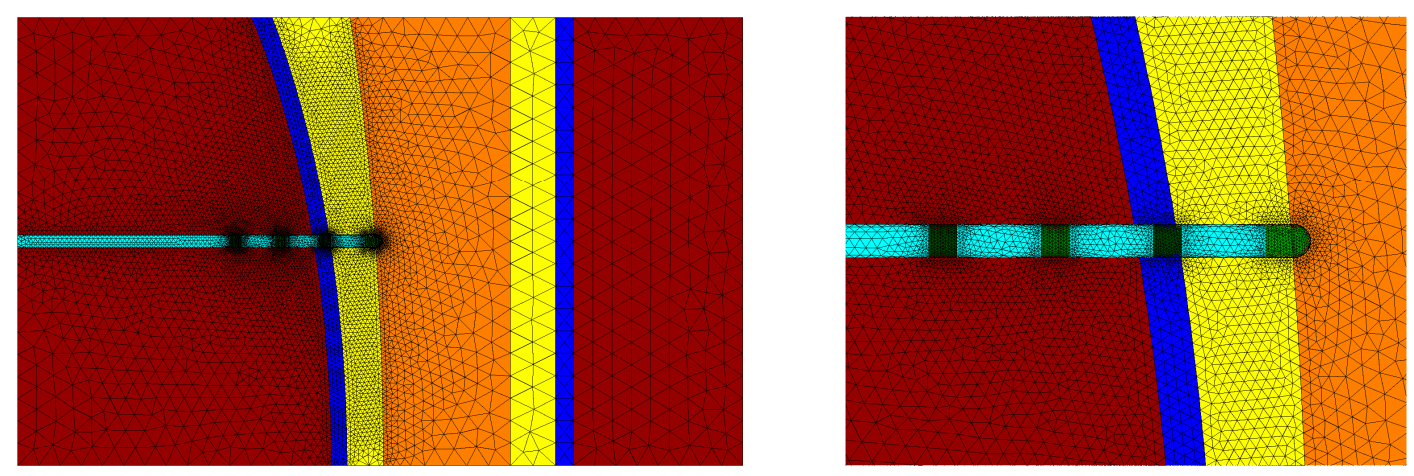

Figure 4.7: Example meshes of a probe entering a bone from the surrounding tissue. Refinement is specified around the electrodes and tissue interfaces near the probe.

Despite the increased ability to control refinement, the results still show that there are discrepancies between the specified parameters and the resulting meshes. The data in table 4.1 show that the maximum edge lengths specified for each mesh were different from the actual maximum edge lengths. As the node density was increased near the electrodes, in Netgen the balance point did not always shift towards the electrode as the maximum node density was not always higher despite specifying a smaller mesh size. In Gmsh the mesh density was closer to the specified values, typically resulting in more nodes in the refined meshes.

The ability to refine meshes selectively in regions where high sensitivity accuracy is required may allow for reduced reconstruction error while reducing meshing times. 
As more electrodes are added and the model complexity is increased, we expect that refinement around the electrodes will continue to reduce total sensitivity error. While this technique can give a starting point to determine the refinement requirement on complex geometries, this analysis cannot be directly applied to arbitrary models. The node balance analysis provides a straightforward method to determine the ideal placement for a given number of nodes to reduce model errors.

\subsection{Summary}

In this chapter we examine the requirement for mesh refinement around electrodes in EIT. While it has been recommended that models be refined around the electrodes, where current density and sensitivity are highest, the level of refinement required is poorly understood. Using a set number of nodes, we investigate the optimal distribution between the electrodes and the volume of a model. A balance point is used to measure the difference in node distribution between the electrode and the centre of the model. To calculate this, all nodes contained between the surface of a selected electrode and the centre of the model were identified and the mean position of nodes along the container axis was computed. We compare refinement strategies across commonly used meshing software in EIT and compare the calculated sensitivity error to results on an ultra-fine reference mesh. In a tank model, for a fixed number of nodes, error in the sensitivity calculation is minimized when the balance point of the nodes is $85 \%$ of the tank radius and the node density dissipates evenly from the electrode surface to the centre of the model. Using this method 
sensitivity error was decreased in regions with high sensitivity. This node distribution technique enables the generation of accurate meshes with fewer nodes that can reduce reconstruction error and meshing time. 


\section{Chapter 5}

\section{Custom EIT Meshes}

This work has been presented in part at: the 21st International Conference on Biomedical Applications of Electrical Impedance Tomography (EIT 2021) (Stowe et al., 2021b).

\subsection{Introduction}

One major challenge of EIT imaging is that the geometry of the external boundary where the electrodes are placed is unknown. Medical images of patients (such as CT images) can provide additional spatial information on patient geometry for the external boundary and location of organs. Recent work has shown that using a custom mesh can improve EIT estimates of lung inhomogeneity (Yang et al., 2021) in ARDS patients, but generating custom meshes manually is not always feasible, and automatic segmentation of CT images is not always reliable. This chapter uses diagnostic CT images from 4 subjects to design and test a tool to create custom 
meshes for each patient. Manual segmentation of CT images can be very time consuming, but unsupervised automatic segmentation does not always perform reliably and can result in inaccurate meshes. In this chapter we combine automatic image segmentation with a manual validation stage to identify an accurate boundary of the body surface and lungs. Once the segmentation is verified, a mesh with custom lung regions is generated to allow for EIT reconstruction on a personalized mesh. A personalized mesh is expected to increase spatial accuracy in reconstructed EIT images. Higher spatial accuracy in identified organs and regions of interest could allow for easier isolation of the perfusion signal, and more accurate measures of perfusion and ventilation with EIT. Motivated by the work presented by Yang et al. (2021), the work in this chapter focuses using custom meshes to improve measures of ventilation in the lungs. We hypothesize this might be able to better guide ventilation and improve treatment outcomes for patients with acute respiratory distress syndrome ARDS.

ARDS is a form of respiratory failure caused by widespread swelling and accompanied by an accumulation of fluid in the lungs. ARDS is a challenging disease to diagnose and treat. No gold standard test exists for diagnosis, and few treatments are effective (Pham and Rubenfeld, 2017). The mortality rate is estimated to be as high as $40 \%$ (Abe et al., 2018). Early studies on the pathology of ARDS suggested adjusting positive end-expiratory pressure PEEP to improve oxygenation in patients (Ashbaugh et al., 1967; Petty, 2001), and recent research suggests that treatment strategies to reduce lung injury during ventilation outperform pharmacological interventions (Duggal et al., 2015). Monitoring ARDS patients during ventilation 
is vital to ensure that ventilator-induced lung injury is avoided (Bates and Smith, 2018), but few appropriate techniques are available. Computed tomography (CT) images used for diagnosis are inappropriate for continuous use due to ionizing radiation exposure, and other global parameters of lung function may not give an accurate estimate of lung homogeneity (Zhao et al., 2009).

EIT was proposed as a monitoring technique for ARDS patients since it is noninvasive and can safely monitor and image the lungs in real time (Denaï et al., 2010. Frerichs et al., 2017). One of the most useful parameters to classify lung ventilation with EIT has been global inhomogeneity (GI) (Hochhausen et al., 2019\} Hough et al., 2016; Hsu et al., 2017; Humphreys et al., 2011, Sribar et al., 2020; Zhao et al., 2012). GI has been identified as a clinically useful parameter to monitor ventilation (Frerichs and Becher, 2019).

The largest issue with using EIT for regional ventilation monitoring is that incorrectly modelling the boundary can introduce large artefacts in reconstructed images (Grychtol et al., 2012). The correct lung boundary is also required to calculate metrics like GI index (Zhao et al., 2009). Incorrectly modelling the boundary and lung area when investigating regional homogeneity can also lead to an incorrect GI estimate (Yang et al., 2021). Yang et al. (2021) found that a mesh with custom segmented external and lung boundaries was more sensitive to changes in lung condition using GI compared with generic models. This suggests a custom mesh with more defined lung regions may also improve sensitivity to lung-based pulsatility and improve measures of perfusion. While GI index is a valuable parameter, it does not give a direct indication of the spatial information in EIT reconstructions. In this 
chapter the centre of ventilation is used instead to compare between EIT recordings and the ventilated region extracted from CT images. This metric is used to determine if a ventilation parameter measured on a custom mesh can be more accurate than when generic meshes are used.

Custom meshes have been created by segmenting the boundaries of lungs and organs to monitor ARDS (Yang et al., 2021), but automatic segmentation of the lung region for a large number of ARDS patients is a challenging problem. Typically, ARDS patients will have a combination of lung swelling, fluid build-up in the lungs, and lung collapse; this obscures the true boundary of the lungs. Figure 5.1 depicts unprocessed CT slices of the $4^{\text {th }}$ intercostal space from 4 subjects with ARDS. This figure highlights the challenges of automatically segmenting lung tissue across several subjects. In some CT images, the arms obscure the external boundary; in others, partial or total occlusions mask the true lung boundary.
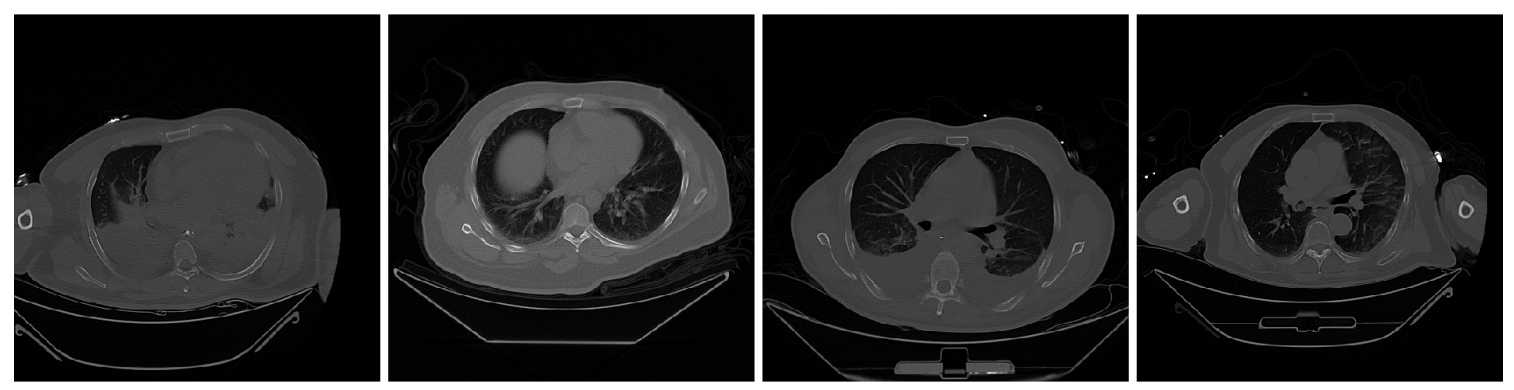

Figure 5.1: This figure shows raw CT data from 4 subjects taken at the $4^{\text {th }}$ intercostal space. These images highlight the challenges with automatic segmentation of the lung and external boundary. In some subjects the external boundary is obscured by the arms, and in other subjects the true lung boundary is masked by occlusions.

This chapter introduces a tool that enables automatic segmentation of the lungs and exterior boundary, with manual correction to facilitate individualized bedside 
monitoring and guide treatment of ARDS. This tool processes and automatically segments the external and lung boundaries in diagnostic CT images, then presents them for manual correction to create custom EIT models. The goal of this chapter is to generate custom models that improve reconstruction accuracy in the lung regions. These models may facilitate real time, individualized ventilation monitoring for ARDS patients.

\subsection{Methods}

This section presents the methodology for:

- Automatic segmentation of diagnostic CT images (section 5.2.1)

- Design of a manual segmentation correction tool (section 5.2.2)

- Mesh generation (section 5.2.3)

- Comparison of ventilation centre of mass between generic and custom EIT models (section 5.2.4) 


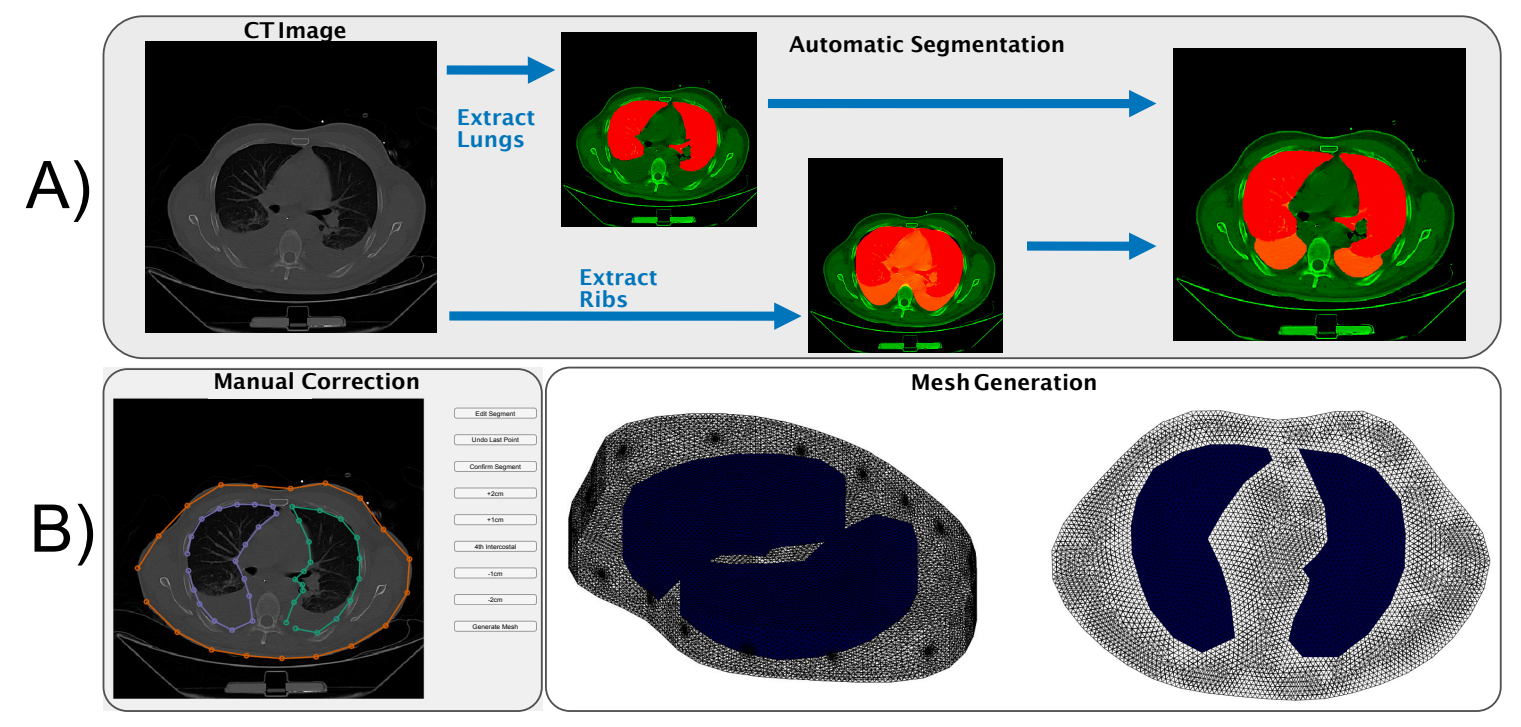

Figure 5.2: An overview of the segmentation and editing process showing: A) A sample raw $\mathrm{CT}$ which was thresholded, scaled and adjusted over several adjacent slices to identify the lung regions and an enclosed rib area, and the resulting lung estimate; and B) A screen capture of the manual mesh correction process and 2 views of the generated mesh.

Figure 5.2 shows a summary of the mesh generation process from raw diagnostic CT data to a custom mesh based on the geometry of the patient. First, CT images are acquired and automatically segmented. Then they are verified or corrected, and a custom mesh is created. Matlab 2021a (Mathworks, USA) with the image-processing toolbox was used for the image segmentation and manual correction interface. Mesh generation and reconstruction was performed with EIDORS 3.10 (Adler et al., 2017a) using Matlab 2019b. At the time of writing Matlab 2019b was required to use certain EIDORS functionality, but Matlab 2021a provided some additional features that improved responsiveness of the graphical user interface GUI 


\subsubsection{Automatic Segmentation of the Thorax}

Automatic segmentation of lung regions in ARDS patients is challenging due to the variability in lung tissue intensity, and the presence of fluid or collapsed lung regions. In some patients the lung regions were not visible in the image and even manual correction of the segmentation required approximation. This project uses an automatic technique to identify the chest cavity using several adjacent vertical CT images to locate the ribs. Even when no lung is visible in the image, this produces a starting point close to the expected boundary which reduces correction time. This tool is designed to approximate the true lung boundary as closely as possible to simplify manual correction if the lungs cannot be segmented. If no ribs or lung are detected, ellipses are placed within the boundary close to the expected lung location that can be corrected manually.

The automatic segmentation step uses a diagnostic CT image with the slice corresponding to the $4^{\text {th }}$ intercostal space identified. To begin segmentation, the user must select the series of CT images they wish to segment, and the frame number that

corresponds to the 4 th intercostal space. For the 4 test subjects, the $4^{\text {th }}$ intercostal space was identified manually using 3D Slicer 4 (Fedorov et al., 2012). This location corresponded to the electrode belt placement for EIT measurements.

A detailed algorithmic outline of the automatic segmentation process is presented in appendix $\mathrm{B}$. 


\subsubsection{External Boundary}

The boundary segmentation steps are shown in figure 5.3 . To segment the boundary, the selected raw $\mathrm{CT}$ slice (A in figure 5.3) was adjusted so that the intensity of the lung tissue was 0 and the maximum image value was 1 (B in figure 5.3). Next the image was eroded and reconstructed to remove small features and retain large structures (C in figure 5.3). Finally, the image was binarized using a threshold of 0.5 (D in figure 5.3), and holes were filled (E in figure 5.3), to give the final boundary ( $\mathrm{F}$ in figure 5.3). 


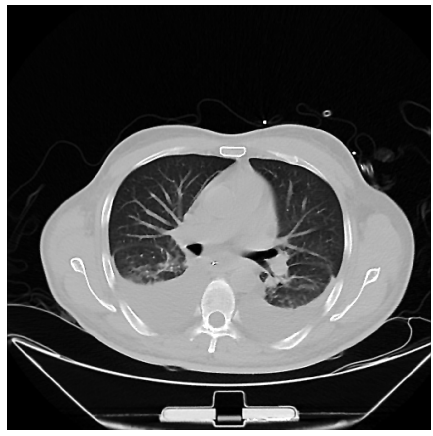

(A)

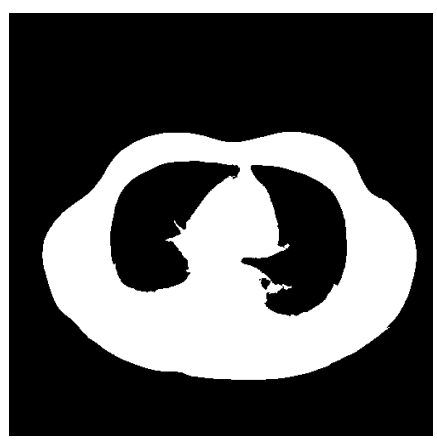

(D)

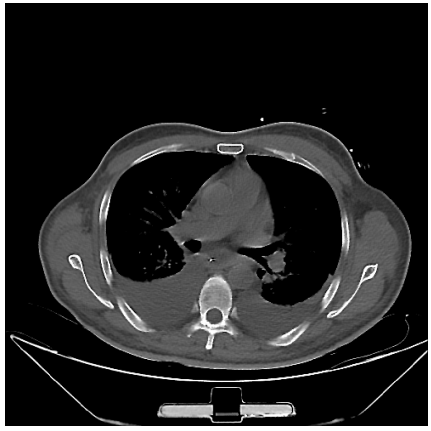

(B)

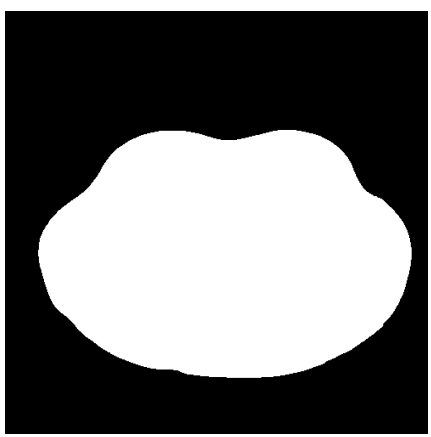

(E)

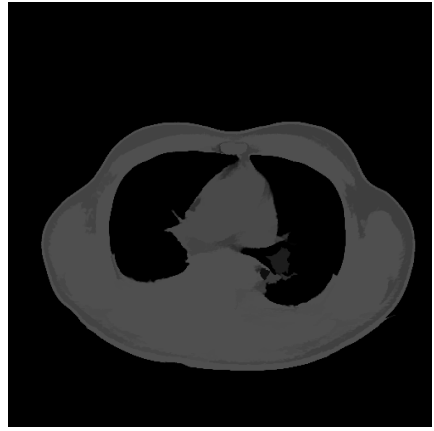

(C)

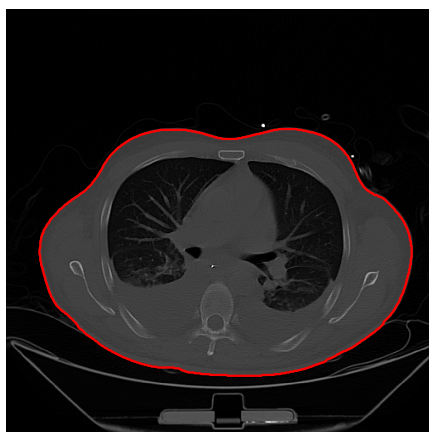

(F)

Figure 5.3: This figure presents the external boundary segmentation process. First, a raw CT image of the 4 th intercostal slice (A) was adjusted based on the lung density (B), eroded and reconstructed $(\mathrm{C})$, then binarized (D) and filled to give the final boundary (F).

\subsubsection{Chest Cavity}

To segment the chest cavity, 7 slices above and below the $4^{\text {th }}$ intercostal space were used, giving 15 slices to segment. To begin, the external boundary of the selected slice was used (segmented using the method in section 5.2.1.1). The initial boundary (A in figure 5.4 was eroded to form a mask from the shrunken boundary shape (B in figure 5.4). This mask was used on a binarized image (threshold of 0.5 ) to extract bones and bright objects from within the thorax ( $\mathrm{C}$ in figure 5.4). The image was 
closed to fill holes and identify bones (D in figure 5.4). Next all 15 adjacent slices were superimposed to combine all rib locations that occurred in more than one slice (E in figure 5.4). To fill holes in the ribcage, a rectangular element with a height of 5 pixels and width of 50 pixels was used to close the top half of the image ( $F$ in figure 5.4). A final thickening operation was performed to ensure continuity ( $\mathrm{G}$ in figure 5.4). All objects connected to the boundary were removed to give the final chest cavity segmentation ( $\mathrm{H}$ in figure 5.4). 


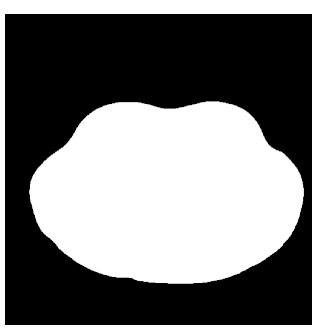

(A)

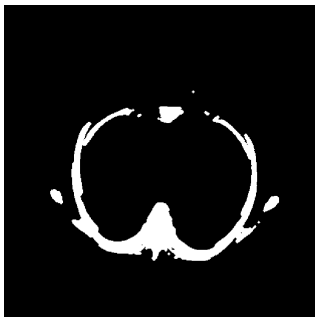

(E)

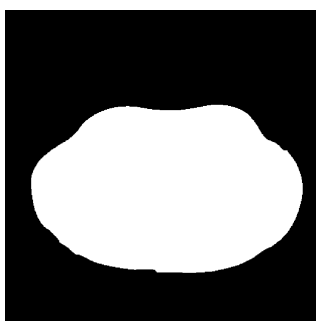

(B)

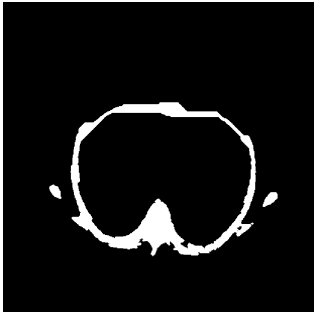

$(\mathrm{F})$

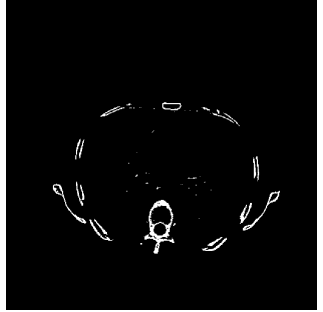

(C)

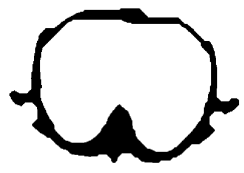

(G)

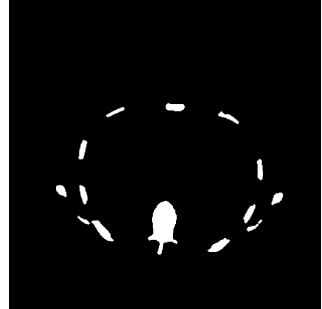

(D)

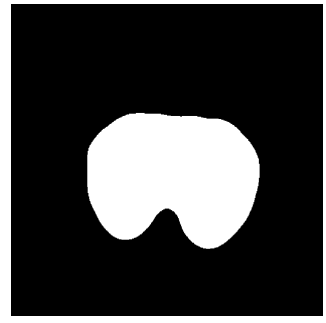

$(\mathrm{H})$

Figure 5.4: The chest cavity segmentation process. For each of the 15 selected slices the initial external boundary (A) was eroded to form a mask from the shrunken boundary shape (B). This mask was used on a binarized image to extract bones and bright objects from within the thorax $(\mathrm{C})$. The image was closed to fill holes and identify bones (D). Next all 15 adjacent slices were superimposed to combine all rib locations that occurred in more than one slice (E). To fill holes, a rectangular element with a height of 5 pixels and width of 50 pixels was used to close the top half of the image (F). A final thickening operation was performed to ensure continuity $(\mathrm{G})$. All objects connected to the boundary were removed to give the final chest cavity segmentation $(\mathrm{H})$.

\subsubsection{Lungs}

The lung segmentation was required to work in patients with ARDS and give an approximate lung boundary when the lungs were potentially collapsed or filled with fluid. To achieve this, a rough lung estimate based on the chest cavity was used together with a segmentation of the ventilated lung. The segmentation process started 
with a segmentation of the chest cavity (A in figure 5.5). First, an estimation of the lung region was made by placing an ellipse in the centre of the chest cavity segmentation at the thinnest central point (B in figure 5.5). Next a ventilated lung estimate $(\mathrm{C}$ in figure 5.5 was made by inverting the binarized image from the external boundary segmentation (D in figure 5.3). Finally, a complete lung segmentation was generated by removing any part of the simple lung estimate (B in figure 5.5) that was within 5 pixels of the ventilated lung region, and combining the two lung estimates with a closing operation. This produced a close estimate of the lung boundary even in cases where little or no lung tissue was visually distinguishable. 


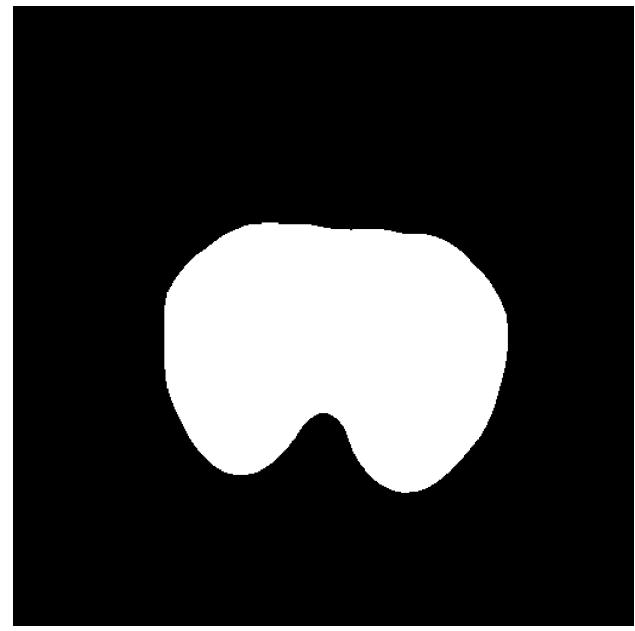

(A)

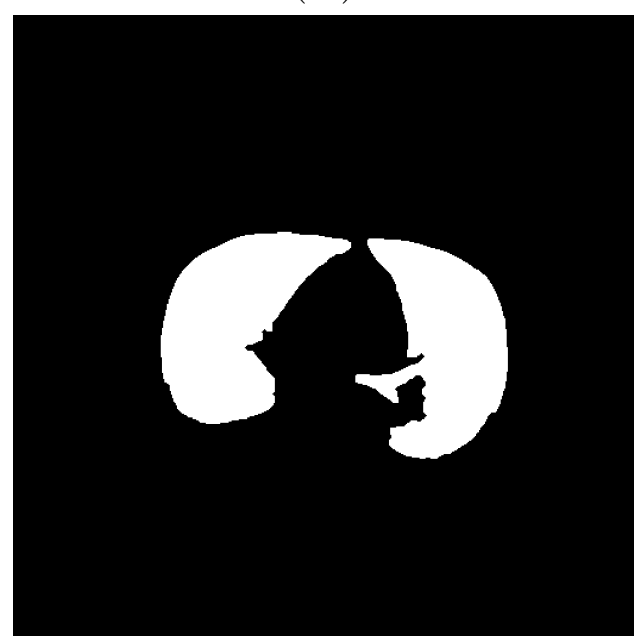

(C)

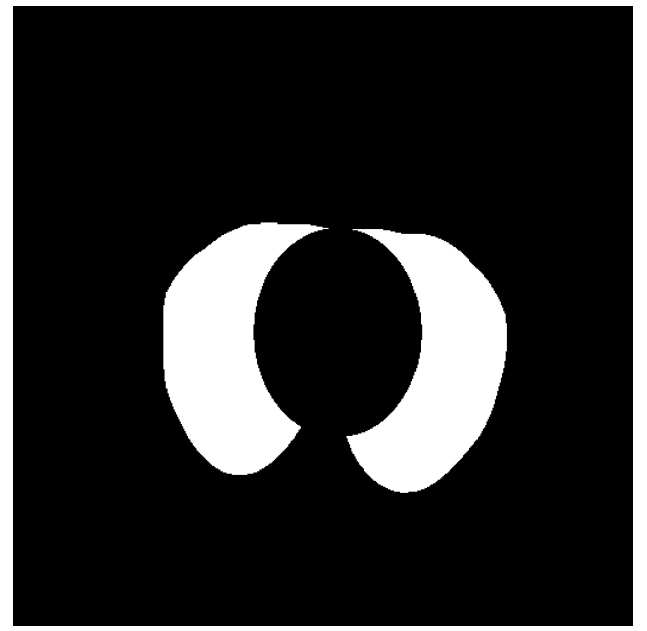

(B)

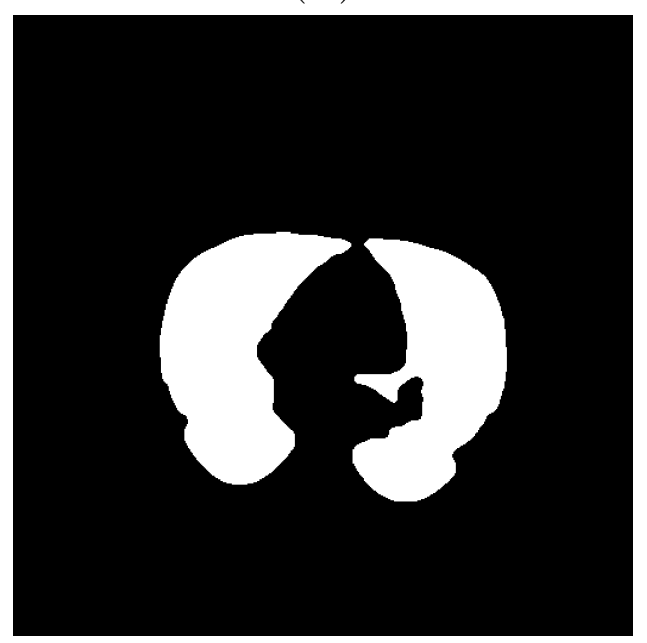

(D)

Figure 5.5: The figure shows the lung segmentation methods. An approximation of the lung boundary was generated using the chest cavity segmentation (A) with an ellipse to remove the heart region $(\mathrm{B})$. This was combined with a segmentation of the ventilated lung region $(\mathrm{C})$ to give a total lung estimate $(\mathrm{D})$. 


\subsubsection{Manual Segmentation Correction}

Although the automatic segmentation was carefully designed to accurately segment the lungs, there were often cases where manual correction was required. To ensure accurate models were generated, an interface for manual correction was created. This tool allowed the user to correct the boundary for a selected CT slice. Sixty control points were overlaid on the CT slice to facilitate correction; Twenty points for each of the lungs and for the external boundary.

The segmentation correction tool was designed to save the segmentation with information on the selected image sequence and frame of the $4^{\text {th }}$ intercostal space for reference. The segmentation correction also allowed users to load previously saved segmentations, and correct them or overwrite them completely. An example of the setup, and available input for the segmentation correction GUI is shown in figure 5.6. To make segmentation simpler, all data such as the frame of the $4^{\text {th }}$ intercostal space was saved after the initial data loading stage. Even if the segmentation was not completed, the user did not have to re-enter patient details when the CT was next loaded. An option was also added to manually input an adjustment value for the automatic segmentation of the ribs. If no ribs were detected during automatic segmentation, an adjustment value could be added to increase or decrease the image binarization threshold. This was not needed as the ribs were successfully identified in all test patients. The software was also designed to give informative error messages to enable troubleshooting, letting the user know if no ribs or no lungs were detected. 


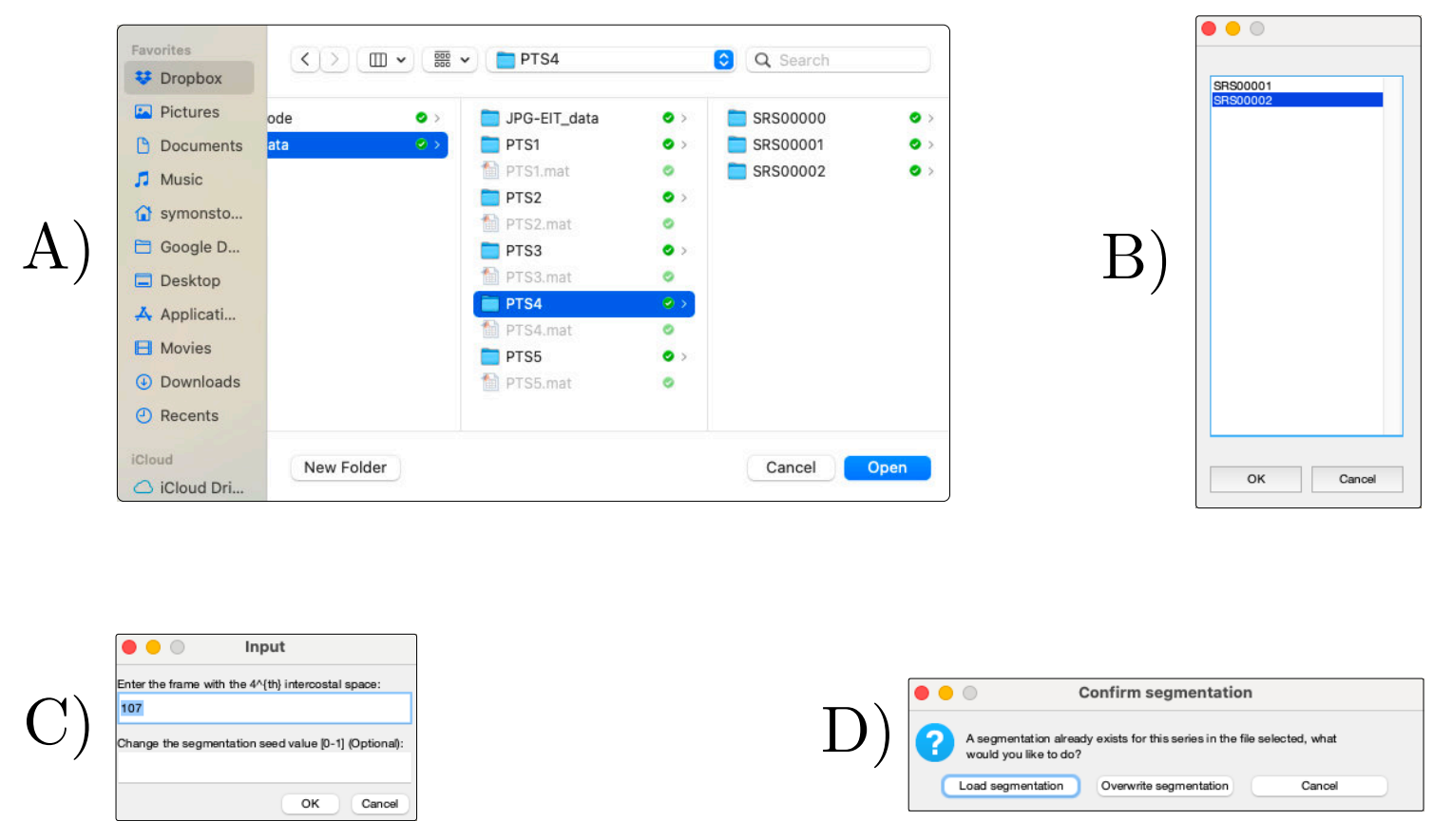

Figure 5.6: The data loading stages for the manual segmentation program are shown above. A) A folder of patient data is selected. B) The desired CT data series is selected. C) The initial segmentation frame of the $4^{\text {th }}$ intercostal space is loaded from the patient settings file or input by the user. Optionally, an adjustment to the initial segmentation thresholding value can be input if the segmentation steps failed to locate ribs in the $\mathrm{CT}$ image. D) If an existing segmentation was saved, the user is asked before a new segmentation is made.

If an error was made during manual correction, the user could undo the last change or revert the selected point to the original location. Keyboard shortcuts were assigned to each of the functions to reduce the time required to segment each slice.

Figure 5.7 shows an example of the Matlab GUI for manually correcting the automatic segmentation. To segment, the user would click Edit Segment then select and move each point that required correction. The first click by the user selected the nearest point, and the subsequent click placed the point at the new location. Buttons on the side allowed the user to select the vertical CT slice for correction, 
save the segmentation, and generate a mesh.

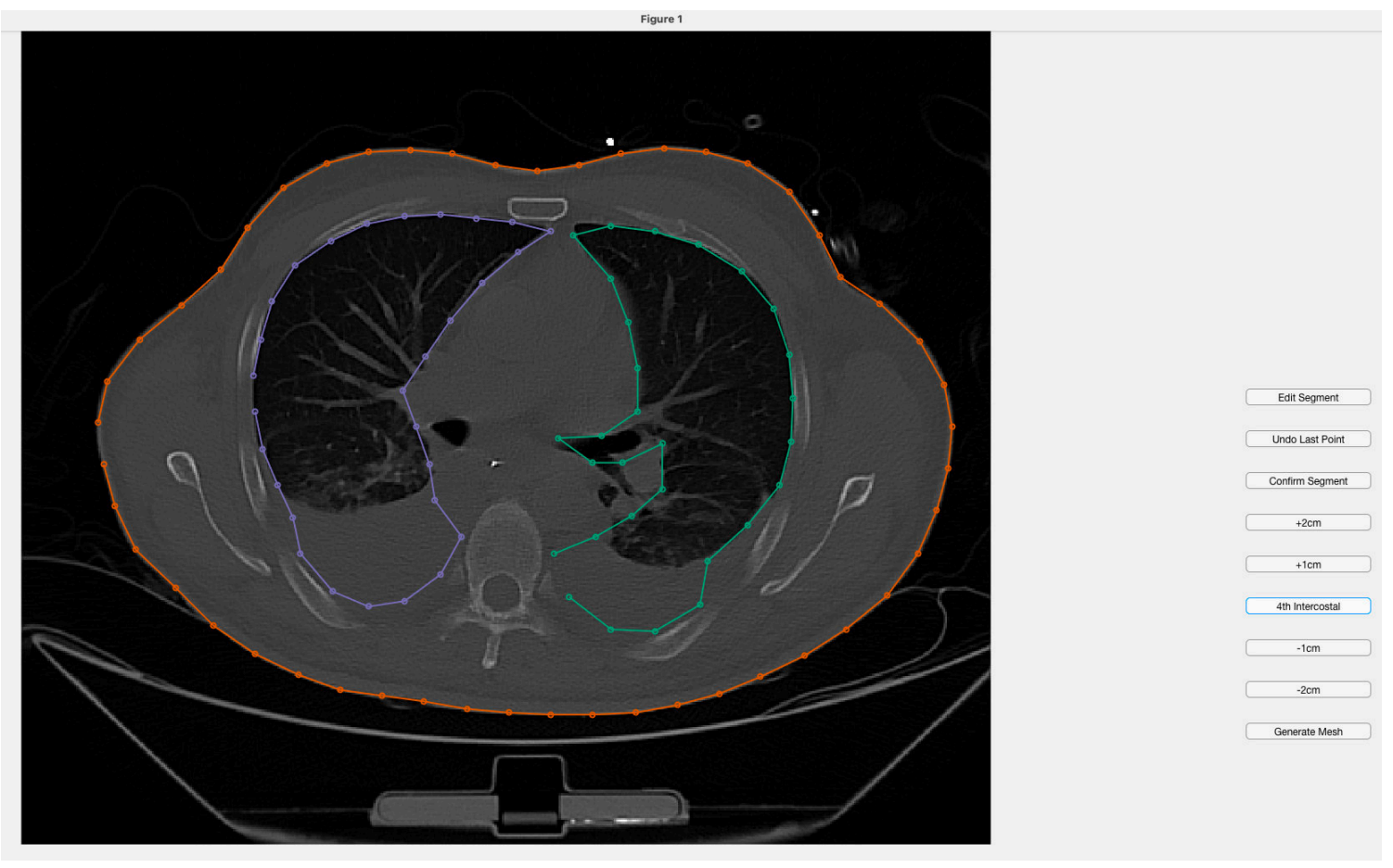

Figure 5.7: An example of a segmentation after being loaded into the segment editor program. The buttons on the right show the options available to the user, and the left shows the CT image for the corresponding slice with the automatic segmentation overlaid.

After moving each point, an arrow was drawn between the original and new location of the point last moved to indicate the change and allow the user to see if the placement was correct. An example of a corrected slice showing the indicating arrow is shown in figure 5.8 . 


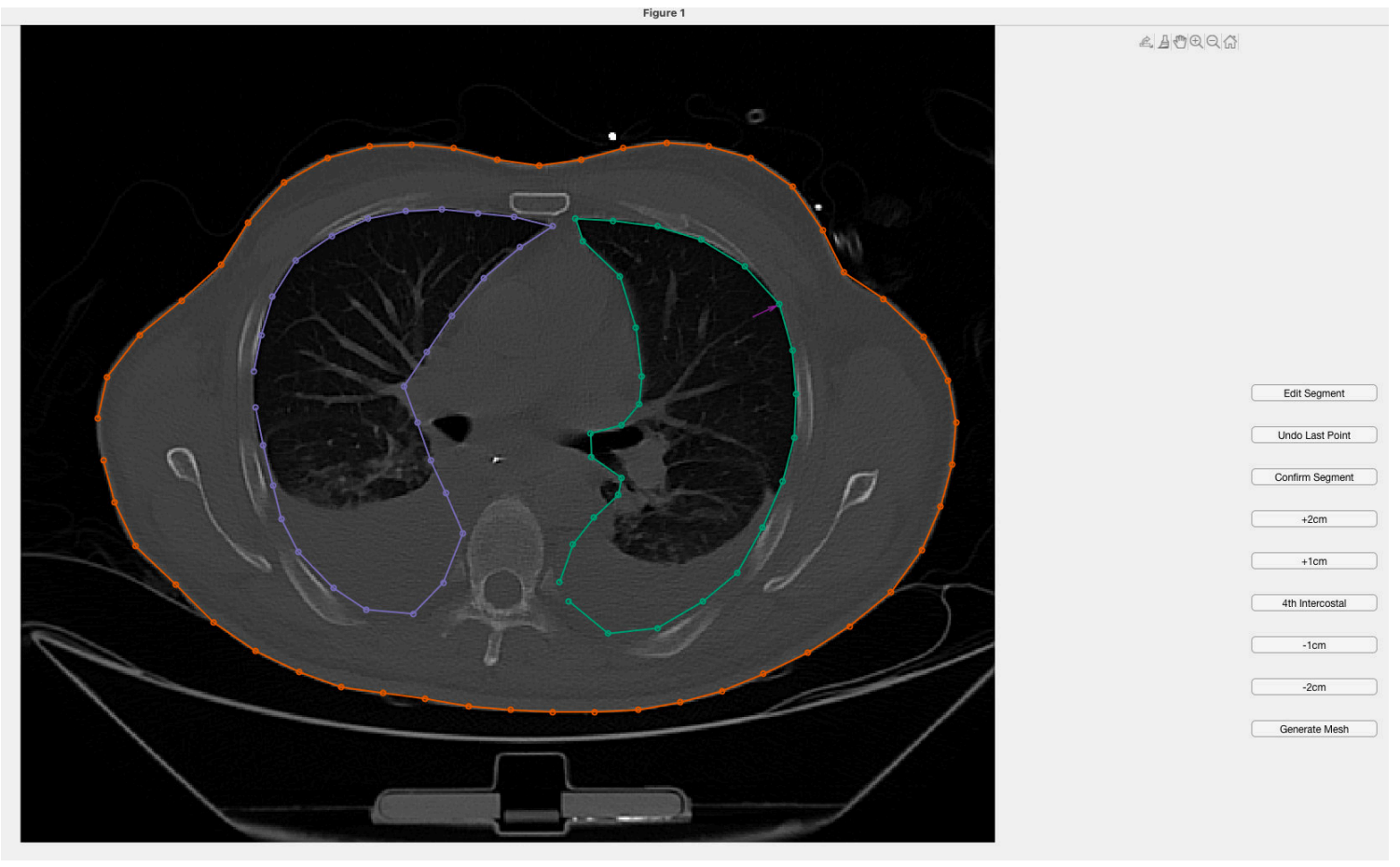

Figure 5.8: An example of a manually corrected segmentation with the arrow indicating the initial and new location of the last moved point. The selected point was moved for illustrative purposes and was not based on the initial segmentation.

The end result was a GUI that allowed for correction and verification of the automatic segmentation results. Spatial information from the DICOM image was also saved so that meshes could be designed to have the correct dimensions and be correctly configured to the patient's size.

\subsubsection{Mesh Generation}

There is currently no available tool to automatically generate $3 \mathrm{D}$ meshes for EIT image reconstruction from a set of points, so the model was simplified using an extrusion technique. The mk_extruded_model function (Grychtol and Adler, 2013) 
in EIDORS v3.10 (Adler et al., 2017a) was used to extrude the boundary of a single segmentation to a height of $20 \mathrm{~cm}$. The segmentation corresponding to the $4^{\text {th }}$ intercostal space was used for extrusion. The lungs were specified 2 ways, the first method extruded 2D lung boundaries to form a 3D lung region, and the second specified a 3D lung boundary that varied over the height of the model based on the segmentation results. To create an accurate 3D lung, we selected all elements of the extruded mesh contained within an alpha shape representation of the lung segmentation with an alpha value of $2 \mathrm{~cm}$.

To generate a generic mesh for comparison the mk_library_model function in EIDORS was used which has available geometry for a cylindrical model with lung regions and a generic model of a human thorax. The conductivity of the lung regions was set to $70 \%$ of the background model conductivity.

All images were reconstructed using GREIT for 2D imaging (Adler et al., 2009). For the GREIT reconstructions the noise figure weighting was set to 0.5 , and 500 targets with a radius of $5 \mathrm{~cm}$ were used for training.

\subsubsection{Evaluation on ARDS Patients}

Diagnostic CT images were acquired from the intensive care unit (ICU) at the Sichuan Provincial People's Hospital in Chengdu, China. The research was approved by the Ethics Committee of Sichuan Academy of Medical Sciences \& Sichuan Provincial People's Hospital (protocol \#201617).

Diagnostic CT data was acquired from 4 male patients aged 39-74 who were diagnosed with ARDS. EIT data were recorded using the Draeger EIT system on all 
patients with a $2 \mathrm{D}$ arrangement of 16 electrodes placed at the $4^{\text {th }}$ intercostal space. All patients were mechanically ventilated at the time of recordings, but the exact ventilation parameters are unknown.

To evaluate the effect of the custom models on lung sensitivity, the centre of mass was computed for both the ventilated region detected in CT images, and the breath volume reconstructed in $\mathrm{CT}$ images. The centre of mass was calculated from EIT images by removing all positive changes in the image, then locating the centre of mass of all pixels that were greater than $50 \%$ of the ventilation signal amplitude. The breath used for the calculation was an ensemble average of all breaths in the recording. The error in the centre of mass was calculated between the EIT reconstructed breath and the CT segmentation, in number of pixels. In this chapter a pixel represents between $0.5-2 \mathrm{~cm}$ depending on the patient size.

\subsection{Results}

The goal of the automatic segmentation algorithm was to generate segmentations that were accurate and could be manually verified. Figure 5.9 shows the result of the automatic segmentation regions. The red overlay indicates the healthy lung segmentation, and the orange overlay indicates regions that were added using the estimation method. The lungs were examined visually to determine how much manual correction was required. During testing we found this level of accuracy was sufficient to complete manual segmentation correction for each subject in under 1 minute. 


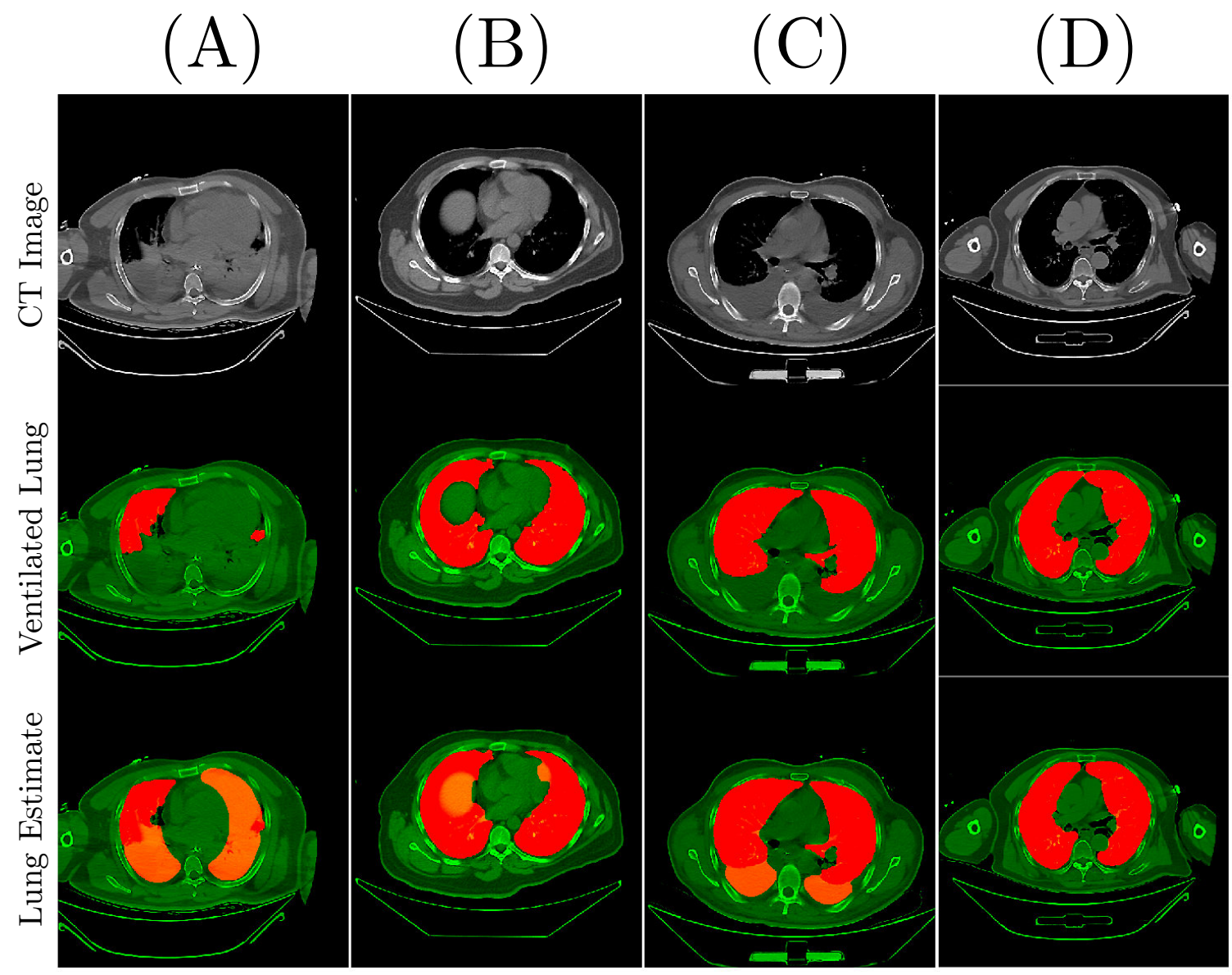

Figure 5.9: This figure shows the results of the automatic segmentation algorithm. The letters A-D represent the 4 patients. Patient A had a significant portion of the lung that was not ventilated, so an estimate was required to obtain lung regions. The ventilated lung estimate is shown in red on the $2^{\text {nd }}$ and $3^{\text {rd }}$ columns. The total lung area determined using the chest cavity and ventilated lung area is shown in the bottom row.

The segmentation results show good identification of the ventilated lung region from the automatic segmentation. The estimated lung regions also helped to give starting approximations for the lungs to decrease required manual correction time when there was limited ventilated lung in the available CT image. Sample meshes generated from the segmented images are shown in figure 5.10 for subject $\mathrm{C}$ (in 
figure 5.9.
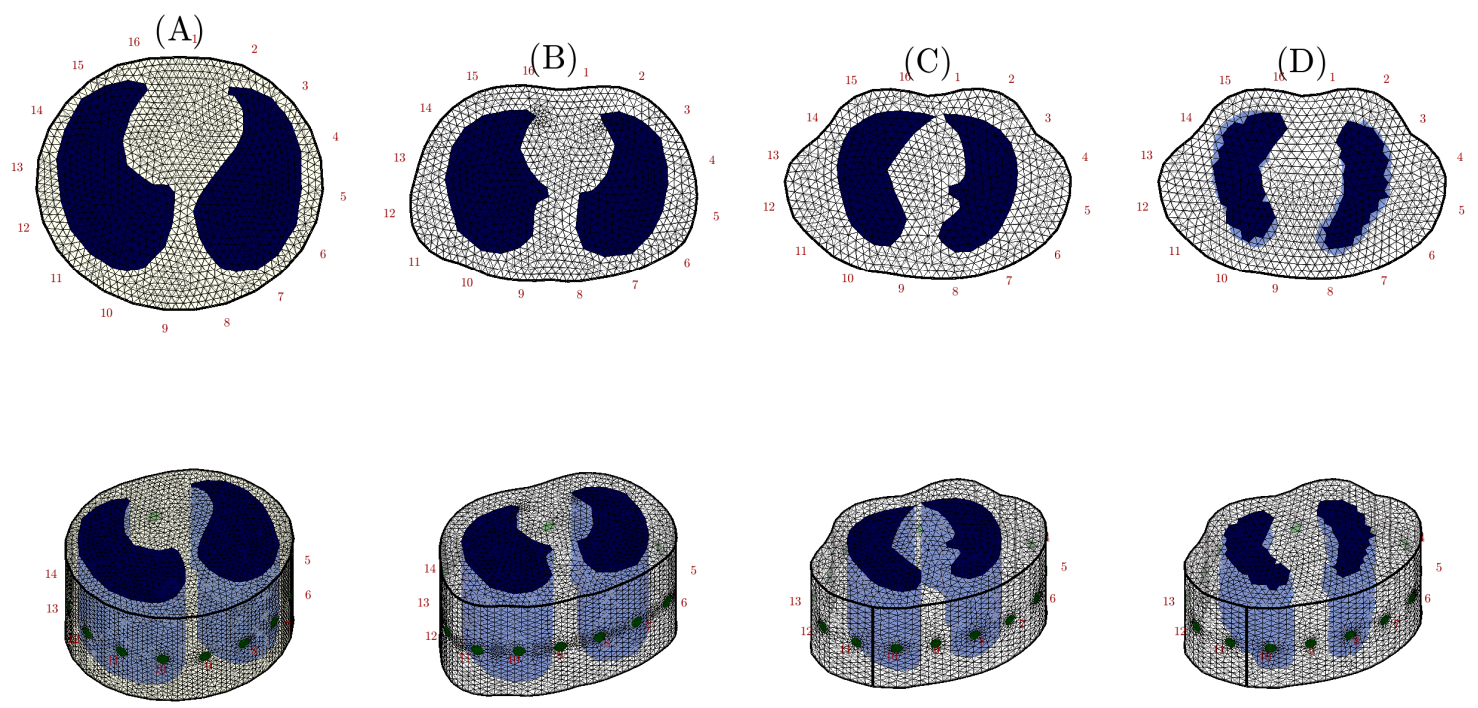

Figure 5.10: Example meshes and electrode locations for subject $\mathrm{C}$ (from figure 5.9). Model (A) is cylindrical with lung regions. Model (B) uses the generic chest model from EIDORS. Models (C) and (D) use the custom segmentation boundaries. Model (C) uses 2D lung boundaries from the $4^{\text {th }}$ intercostal space extruded for the model height. Model (D) selects all elements are within the 3D lung boundary, as identified by the segmentation results.

For each patient there was a single EIT recording ranging from 30 - 60 seconds in length. The ventilation pattern from the CT image was compared to the reconstructed ventilation on 4 types of models. The 4 types of meshes compared are pictured in figure 5.10. The first mesh was cylindrical with approximate lung regions, the second mesh was a generic model for an adult thorax, and the final two meshes were created from the segmentations. To compare the performance across all models, the centre of mass was compared between the CT-imaged ventilation and the EIT-imaged ventilation. The mesh with 3D extruded lung regions resulted in a 
mesh that most closely matched the segmentation, since selecting a complex 3D lung region caused some of the spatial information from the lung segmentation to be lost.

Reconstructed images in figure 5.11, figure 5.12, figure 5.13 and figure 5.14 show a small difference in ventilated regions and centre of mass between custom and generic models. There is a noticeable change in ventilation distribution using the circular model, but the generic thorax model and the custom models show very similar results. There is also a slight shape difference in the ventilation distribution between the generic thorax model and custom models, but there is little measurable difference.

(A)
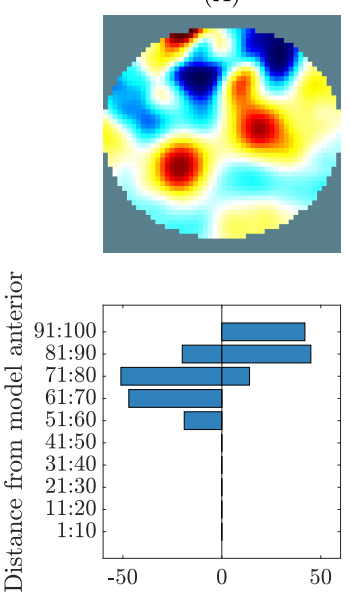

(B)
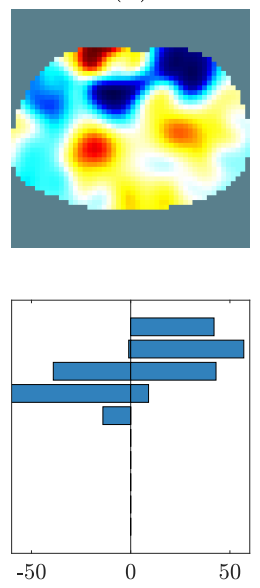

(C)
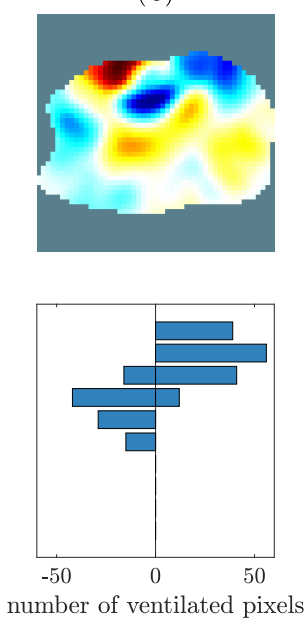

(D)
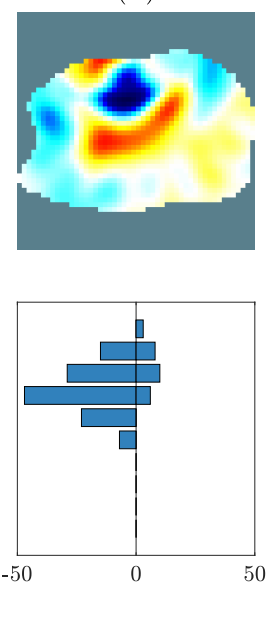

(E)
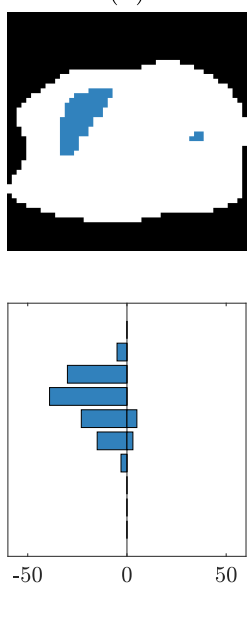

Figure 5.11: Example ventilation distribution for subject A (from figure 5.9). Column A and B are the generic models from the EIDORS library. Columns C and D show the custom models with extruded 3D lungs (C) and complex 3D lung regions (D). Column E shows the distribution of the ventilated lung as segmented from the CT image. 
(A)
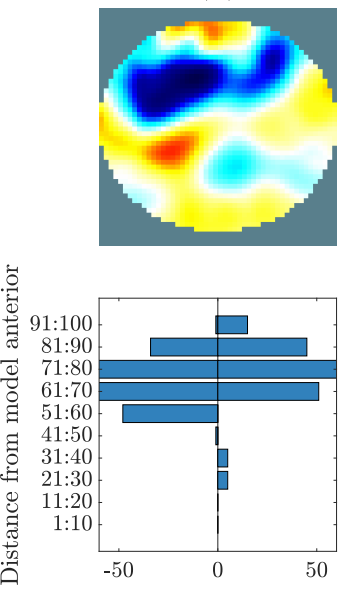

(B)
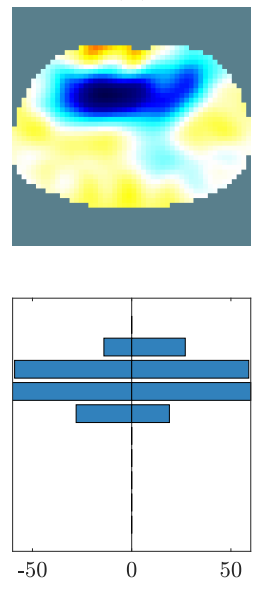

(C)
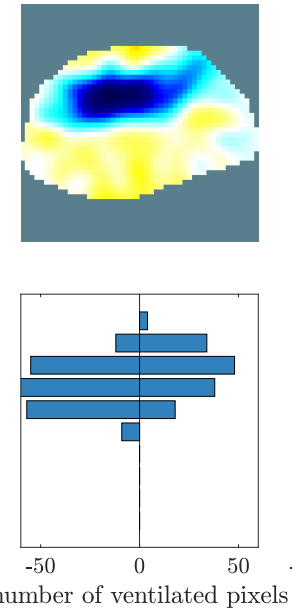

(D)
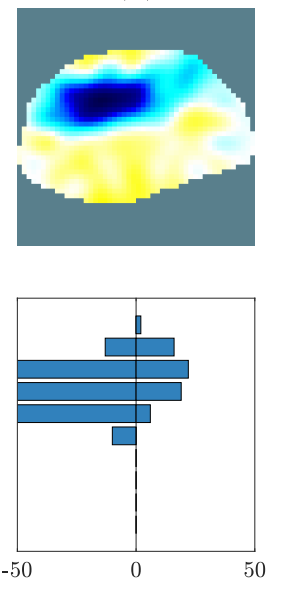

(E)
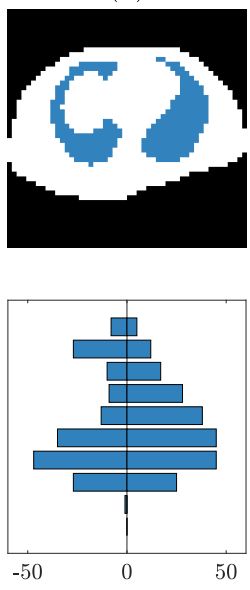

Figure 5.12: Example ventilation distribution for subject B (from figure 5.9). Column A and $\mathrm{B}$ are the generic models from the EIDORS library. Columns $\mathrm{C}$ and D show the custom models with extruded 3D lungs $(\mathrm{C})$ and complex 3D lung regions (D). Column E shows the distribution of the ventilated lung as segmented from the CT image.

(A)
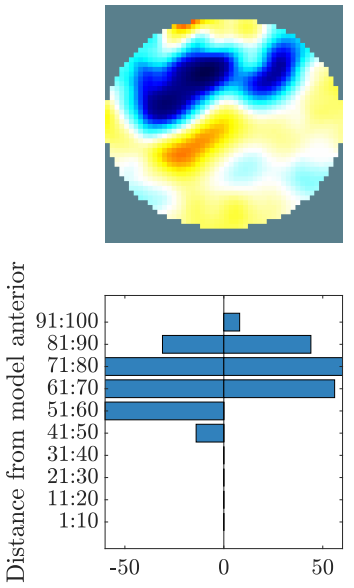

(B)
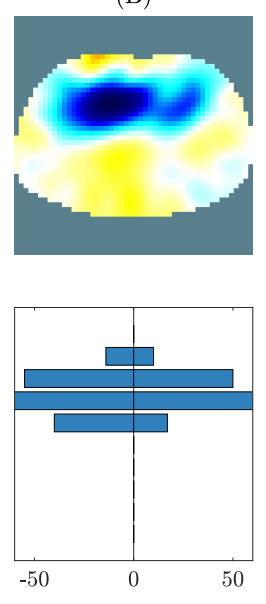

(C)
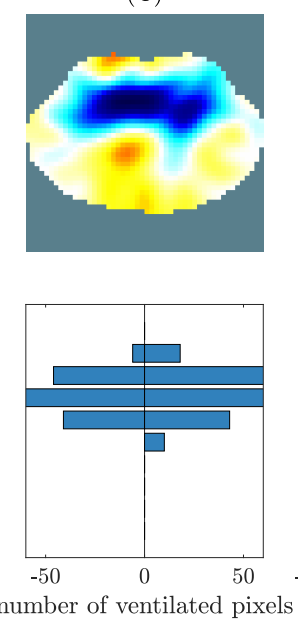

(D)
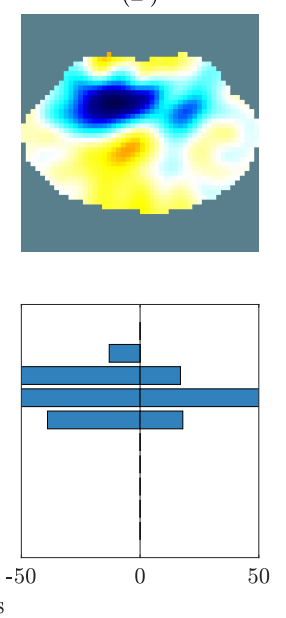

(E)
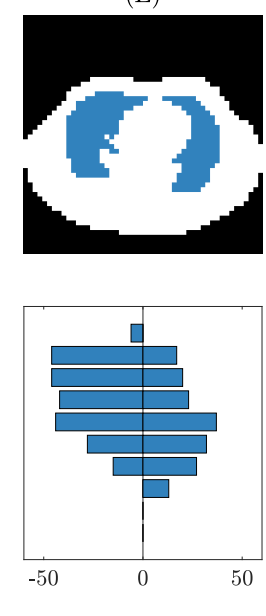

Figure 5.13: Example ventilation distribution for subject $\mathrm{C}$ (from figure 5.9). Column A and $\mathrm{B}$ are the generic models from the EIDORS library. Columns $\mathrm{C}$ and D show the custom models with extruded 3D lungs (C) and complex 3D lung regions (D). Column E shows the distribution of the ventilated lung as segmented from the CT image. 
(A)
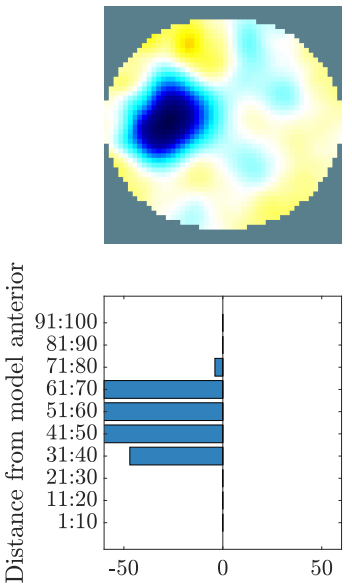

(B)
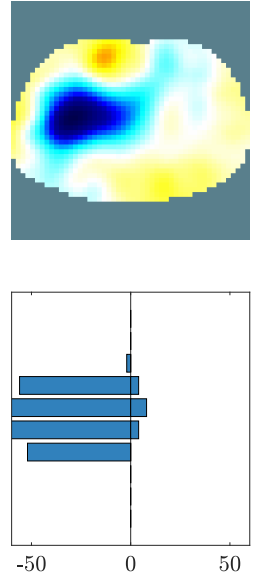

(C)
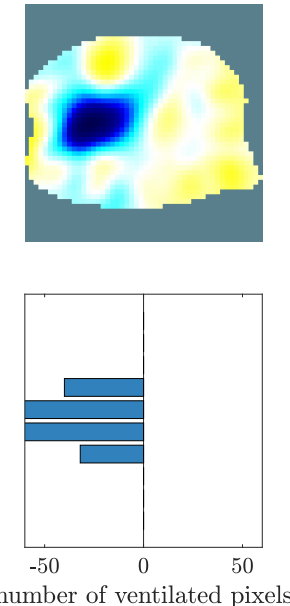

(D)
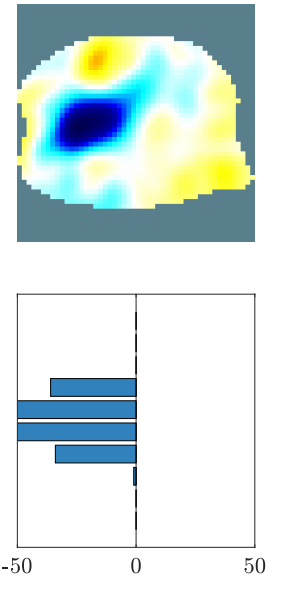

(E)
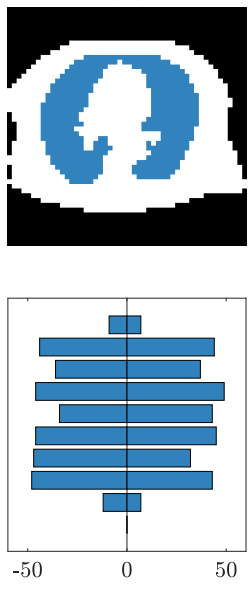

Figure 5.14: Example ventilation distribution for subject D (from figure 5.9). Column A and $\mathrm{B}$ are the generic models from the EIDORS library. Columns $\mathrm{C}$ and $\mathrm{D}$ show the custom models with extruded 3D lungs (C) and complex 3D lung regions (D). Column E shows the distribution of the ventilated lung as segmented from the CT image.

Error in the centre of mass was calculated across all models for each subject as shown in figure 5.15. The error is estimated as the distance in pixels between the centre of mass of the ventilated lung from the $\mathrm{CT}$ estimate and the centre of mass of the reconstructed breath. 


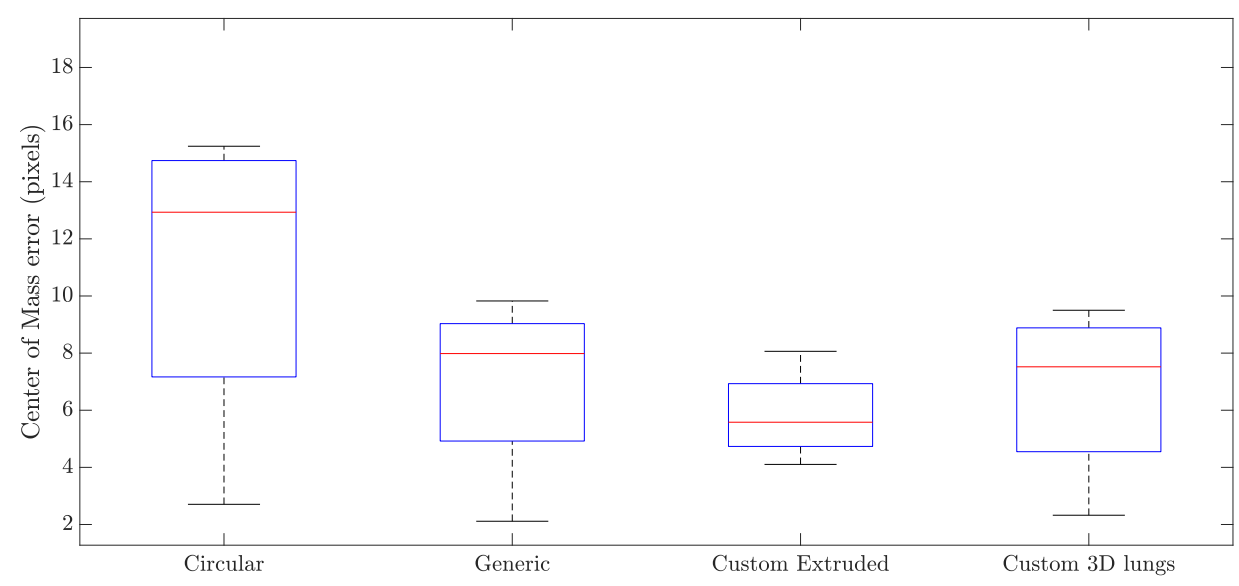

Figure 5.15: This figure shows error in the centre of mass measured in pixels for each of the 4 model types investigated. From left to right are the circular, generic, 3D extruded, and complex 3D lung region models. One pixel represents between $0.5-2 \mathrm{~cm}$ depending on the patient and CT configuration.

Based on this small sample size it seems that the custom extruded models show some improvement over generic EIT models. All body-shaped models performed better than the cylindrical model as measured by error in the centre of ventilation, and there was little difference between the generic model and the model with complex 3D lung regions. There are several potential factors that could contribute to the similarity in performance across most models.

\subsection{Discussion}

The goal of this chapter was to create a tool to automatically segment CT images to improve lung reconstruction accuracy. We believe this may help to enhance individualized ventilation monitoring for ARDS patients. An automatic segmentation 
algorithm was developed to give a baseline segmentation of the lungs which gave an approximate starting point for the true lung boundary. A tool for manual correction was designed that enabled quick, accurate corrections of automatically generated CT images. Segmented images were used to generate meshes with accurate boundary shapes and lung outlines.

Previous studies looked at the effect of model accuracy on GI index and found that a more accurate body surface and lung boundary improved results (Yang et al., 2021). The results presented here show only a small improvement when examining difference in the centre of ventilation. A large sample size is required to determine if the improvement is consistent. One factor that was different between the two studies was the value of lung conductivity. Yang et al. (2021) used a conductivity value of 1.3 times the background conductivity for the lung tissue. This is atypical since the conductivity of lung tissue is considerably lower than other tissues in the body. In this chapter a lung conductivity of $70 \%$ of the background conductivity was used. Using a value of 1.3 times the background conductivity did not decrease the centre of mass error across custom models. There was also a difference in the centre of ventilation error when comparing between both custom models. One potential reason is that when selecting the elements that were contained within the complex 3D lung regions, the boundary of the lungs was distorted slightly and a less accurate representation of the lungs was given. If images were acquired with a 3D arrangement of electrodes, the use of a complex 3D volume to represent the lungs could have more of an effect.

There are several other factors that may contribute to the mismatch between the 
CT ventilation and the EIT imaged ventilation. First, the difference in posture and condition between the CT and the EIT image was not known. The EIT belt was not in position during the CT scan, so there was potential that the patient position and posture were slightly changed. Although this may contribute to a slightly different shape of the thorax, it is not likely to drastically change the boundary of the chest cavity. One of the larger factors is likely the unknown locations of the electrodes. Since the exact location of the electrode is unknown, the 16 electrodes were places evenly around the thorax in the model. Modelling the electrode locations incorrectly can have a large impact on the resulting EIT image, causing artefact and lower reconstruction accuracy (Boyle and Adler, 2011). Since the CT images acquired contain spatial information, there is potential to improve accuracy of the electrode placement on the models. The EIT system that was used has several available belts with different electrode spacing and points of attachment. If the user was able to select the belt used and the electrode spacing in the software, the electrode locations could be more accurately approximated. Alternatively, an image or CT with the electrode belt in place could allow precise estimation of the electrode location and spacing. It may also be feasible to reconstruct the electrode location partially using a movement jacobian (Soleimani et al., 2006), although it is not currently possible to do this with the GREIT algorithm.

In addition to improving the accuracy of electrode placement in the meshes, there is still future development planned for the segment editor and automatic segmentation. Initial tests were done on the $\mathrm{CT}$ and EIT data from 4 patients, and validation across a wider dataset is required to ensure stability of the automatic segmentation 
and ease of editing. Improvements to the appearance of the interface and documentation must also be added before the software is easy to use in a clinical setting. Work is currently underway to obtain several more sets of CT and EIT data to validate the automatic segmentation and manual correction steps. Once the number of patients is increased, the project will use these meshes to build a library of meshes that can be extended for use on patients without CT. A library of meshes coupled with machine learning based on physical attributes could allow for custom meshes to be used on patients even when no CT data is available.

When using the complex 3D lung region model, there was a slight reduction in the amplitude of a conductive artefact that appeared in the centre of the images. It is possible that the 3D aspect of the model allows the $3 \mathrm{D}$ path of the current to be modelled more accurately (Adler and Boyle, 2017), but the extent of the benefit is limited when using a 2D arrangement of electrodes (Grychtol et al., 2016). The segmentation techniques could be used to create an even further customized model with different conductivity regions assigned to fluid in the lungs and collapsed regions. While this could give more spatially accurate reconstructions, we still do not expect a high degree of spatial accuracy using EIT. The advantage of EIT is primarily the high temporal resolution. Additionally, the collapsed regions and liquid in the lung may move over time. Identifying the entire lung region allows us to track changes in impedance within the lung region.

When creating meshes using the ng_mk_extruded_model in EIDORS (Grychtol and Adler, 2013), accurate models with a good representation of the boundary are created, but Netgen occasionally fails to mesh the electrodes on models. We pre- 
sume this is due to irregular and concave curves. This occurred several times on the segmented data, so a more robust meshing or electrode placement technique will be required. Placing complex 3D lung boundaries helped to reduce errors that were introduced by tight corners on lung segmentations, but electrode and boundary meshing errors were still present in this model.

\subsection{Summary}

The diagnostic CT images required for ARDS patients are an excellent source of spatial information about the size and location of organs in a subject. A segmentation and boundary-editing tool was developed to generate accurate meshes of the external and lung boundaries from CT images. The custom meshes with an extruded boundary show a slight reduction in error when comparing centre of ventilation to the CT images. In ARDS patients with very poor lung health, segmentation of the actual lung boundary is challenging. However, with a multi-step process including automatic segmentation and manual verification, we generated accurate customized meshes for a small number of ARDS patients. Small benefits were observed when reconstructing ventilation images on custom meshes, and additional steps to improve the modelled electrode locations could help to further improve reconstructions. Work is currently underway to validate and improve the segmentation tool on a larger dataset of ARDS patients. Methods to reduce the impact of sensitivity to electrode location error on reconstructions, or correctly identify correct electrode locations could help to further improve this technique. 


\section{Chapter 6}

\section{Internal Electrode Sensitivity}

This sensitivity analysis work has been presented in part at: the 42nd Annual International Conference of the IEEE Engineering in Medicine $\mathcal{E}$ Biology Society (EMBC 2020) (Stowe and Adler, 2020).

\subsection{Introduction}

Currently, the most common implementations of EIT in biomedical applications measure voltages and inject currents from the body surface using one or two planes of electrodes. Internal electrodes are not used clinically despite promising simulation studies that have shown improvements in reconstruction accuracy and internal sensitivity in 2D (Nasehi Tehrani et al., 2012a; Nasehi Tehrani et al., 2012b). In practice, EIT images with internal electrodes are challenging to interpret as the measurements are prone to errors due to the high sensitivity near the internal electrodes and the complexities of probe placement and reconstruction (Czaplik et al., 2014). 
As discussed in chapter 3, one of the main challenges of perfusion imaging is the large difference in amplitude between the ventilation and cardiac signals. When using external electrodes, the respiratory amplitude is an order of magnitude larger than the cardiac component. Techniques such as breath holds, induced apnoea (Leathard et al., 1994; Stowe et al., 2019), or injection of a contrast agent (Frerichs et al., 2002) can reduce the impact of the respiratory signal, but they are not feasible for long-term or continuous monitoring. Other methods such as averaging a large number of signals together have been used (Eyüboğlu et al., 1989, Vonk Noordegraaf et al., 1998), but as discussed by Deibele et al. (2008) these do not allow real-time monitoring and may miss sudden changes in perfusion. Filtering techniques have been implemented to help isolate the cardiac component of the signal, but there is occasionally overlap between the cardiosynchronous component and harmonics of the ventilation signal. This makes signal separation more challenging (Leathard et al., 1994, Zadehkoochak et al., 1992).

Internal electrodes have been proposed several times as a method to continuously increase sensitivity in central regions of the chest and improve EIT reconstruction accuracy (Czaplik et al., 2014; Nasehi Tehrani et al., 2012b; Nguyen et al., 2020; Pilkington et al., 1989; Schuessler and Bates, 1995). Past research in 2D identified a sixfold increase in the cardiac frequency component of the EIT signal when 2 of 16 total electrodes were placed in the esophagus or trachea compared to a typical external configuration (Czaplik et al., 2014). However, it is not clear whether the identified increase in cardiac-frequency amplitude stems from pulsatile (motion-based), changes or whether these changes represent perfusion. 
There is potential for clinical use of internal electrodes to monitor ventilation, perfusion and hemodynamic changes in the intensive-care unit (ICU), where patients typically have breathing and feeding tubes in place. EIT applications in development include mechanical ventilation guidance and monitoring (Frerichs et al., 2017), perfusion monitoring (Frerichs et al., 2002; Smit et al., 2003), blood pressure monitoring (Proença et al., 2017, Solà et al., 2011), and cardiovascular output (Braun et al., 2018a). For all of these uses of EIT, internal sensitivity in the centre of a model could provide increased accuracy. In an intensive-care environment where feeding and breathing tubes are used, internal electrodes could provide several advantages without additional invasiveness. It has even been suggested that internal electrodes may replace electrodes on the back, which are challenging to place for critically ill patients who cannot be safely or easily moved (Czaplik et al., 2014). Internal electrodes placed in both the esophagus and the trachea have been used, and both have shown increased sensitivity to cardiosynchronous EIT signals (Czaplik et al., 2014). Esophageal electrodes may be slightly easier to implement clinically as there are fewer hygiene concerns.

Especially while monitoring perfusion where one of the main challenges has been the small amplitude of the cardiac signal (Nguyen et al., 2012), internal electrodes offer potential for improvement. This chapter presents a 3D EIT configuration with internal electrodes that can be used to maintain a high sensitivity in regions with large pulsatile components in the centre of a model.

Some studies have suggested different current injection and measurement patterns to improve sensitivity when using internal electrodes (Nasehi Tehrani et al., 2012a). 
This chapter presents a comparison between an electrode injection pattern to increase internal sensitivity to the standard "skip 4" measurement and injection pattern used in $3 \mathrm{D}$.

The sensitivity benefits of internal electrode imaging in 3D are assessed in simulation to determine the feasibility of using internal electrodes to improve perfusion monitoring. We expect to see that internal electrodes increase sensitivity in 3D configurations as they do in 2D. This chapter provides proof of principle for internal electrode configurations in 3D to improve EIT sensitivity.

\subsection{Methods}

\subsubsection{Tank Model}

This section explains the techniques used to generate 3D meshes with different external and internal electrode configurations on a tank model. Different numbers of electrodes were added to visualize the sensitivity increase and determine if accurate constructions were feasible. To analyze the sensitivity changes due to different electrode configurations finite element models (FEMs) of a cylindrical tank were created with each of the tested electrode configurations. Figure 6.1 shows the four different configurations that were tested: a 2D electrode ring of 32 electrodes; a 3D configuration of 2 layers of 16 electrodes $(3 \mathrm{D}(\mathrm{a}))$; a second 3D configuration of 2 layers of 15 electrodes plus 2 central internal electrodes inline with the electrode planes $(3 \mathrm{D}(\mathrm{b}))$; and a final 3D configuration of 2 layers of 14 electrodes with 4 central internal electrodes evenly spaced between the electrode planes $(3 \mathrm{D}(\mathrm{c}))$. 


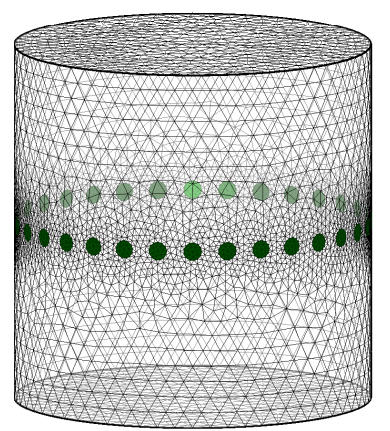

2D

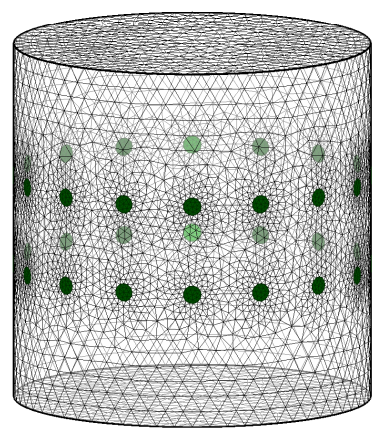

3D(a)

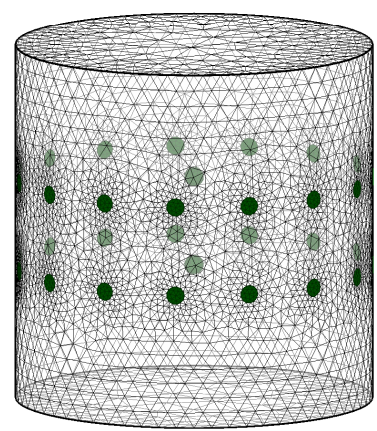

3D(b)

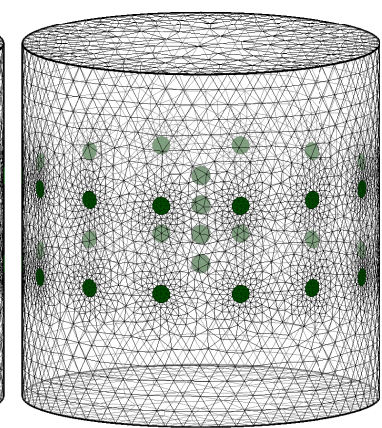

$3 D(c)$

Figure 6.1: 4 configurations of electrodes were tested: 2D) a single ring of 32 electrodes; $3 \mathrm{D}$ (a) 2 rows of 16 external electrodes; 3D(b) 2 rows of 15 external electrodes with 2 internal electrodes; and $3 \mathrm{D}(\mathrm{c}) 2$ rows of 14 external electrodes and 4 internal electrodes.

The tank in the simulations has a height of $2 \mathrm{~m}$, radius of $1 \mathrm{~m}$, and the electrode radius is $0.05 \mathrm{~m}$ for both the round external electrodes and the spherical internal electrodes. In the 3D configurations, the plane separation is $0.5 \mathrm{~m}$ and in all configurations the radial spacing between electrodes is equal. The background conductivity of the tank was $1 \mathrm{~S} / \mathrm{m}$ and the conductivity of the target was $10 \mathrm{~S} / \mathrm{m}$.

When reconstructing images a conductive target was added to the tank at a height of $1 \mathrm{~m}$ at the midpoint of the tank radius. The target object radius is $0.4 \mathrm{~m}$.

\subsubsection{Image Reconstruction}

To generate EIT images from voltage measurements, the 3D GREIT reconstruction algorithm was used (Grychtol et al., 2016). A spherical conductive target with a radius of $20 \%$ of the tank radius was placed midway between the centre and boundary of the tank, in a region with typically low sensitivity. The inverse problem hyper- 
parameter was selected so that in all instances the amount of measurement noise that propagated from the measurements into the final images was equal. 500 training targets with a size of $5 \mathrm{~cm}$ were placed within the model to train the GREIT algorithm.

\subsubsection{Sensitivity Calculation}

The sensitivity is then calculated from the jacobian $(J)$ of the reconstruction matrix as:

$$
S_{i j}=\frac{\sqrt{\sum_{i} \vec{J}_{i j}^{2}}}{V_{i}}
$$

where $V_{i}$ is the volume of each respective voxel.

\subsubsection{Current Injection and Measurement}

For this analysis external electrodes are placed in a "square" pattern (Grychtol et al., 2016) and the internal electrodes are placed from top to bottom.

The current injection pattern used in this analysis was the typical "skip 4" injection pattern that has been shown to yield a good sensitivity distribution in 3D EIT (Grychtol et al., 2016). A new stimulation and measurement pattern is also used in a model with two internal electrodes that increases the number of measurements on the internal probe. This stimulation pattern is consistent with the skip 4 pattern, but for the measurements, every second measurement is replaced with a measurement between the boundary and the internal probe. This results in the same total number of measurements, but many more measurements use the internal probe. This new 
stimulation pattern is shown in figure 6.2. All measurements are made between the top and bottom plane of electrodes.
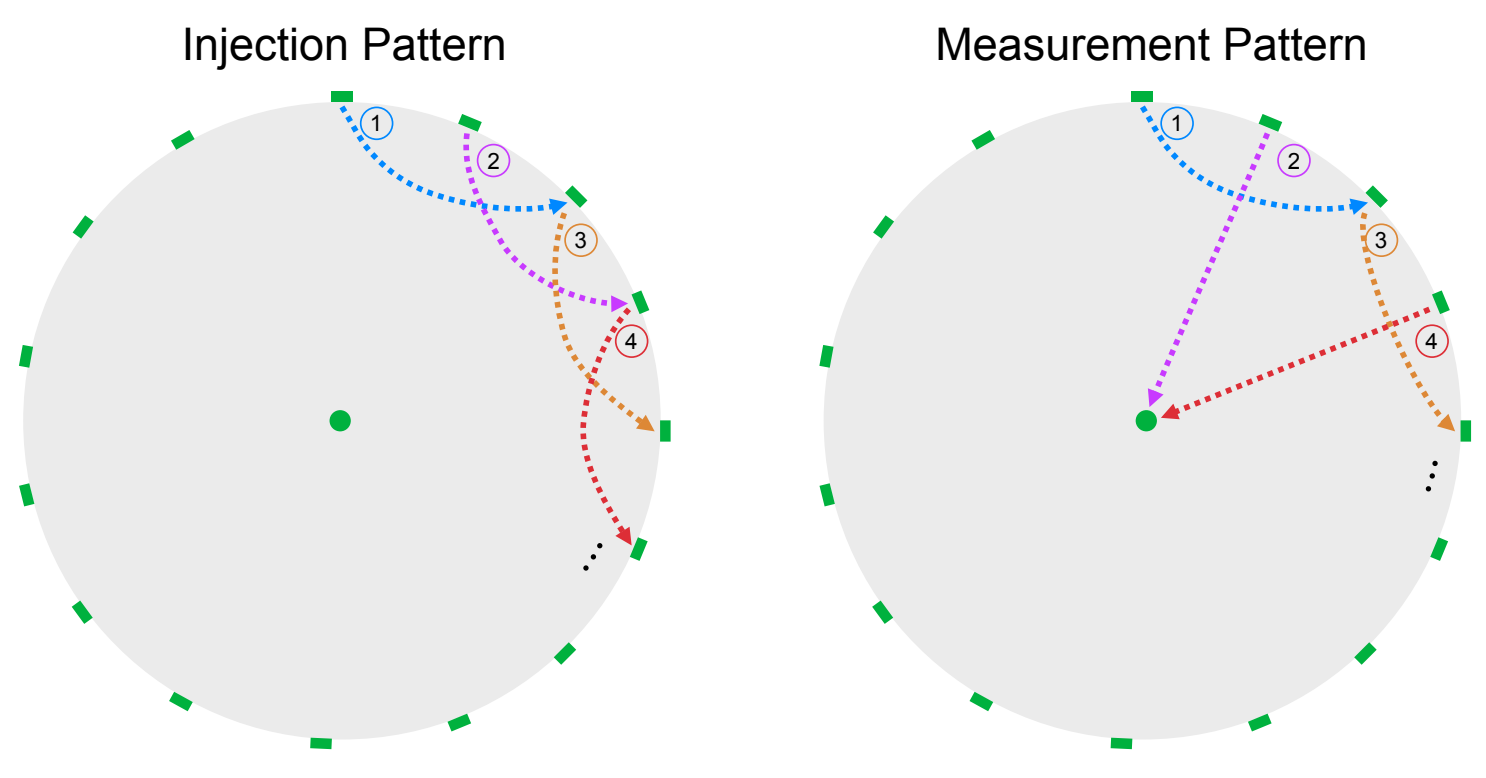

Figure 6.2: A proposed current injection and measurement pattern for EIT imaging with 2 internal electrodes. The injection pattern is a typical "skip 4 " pattern injecting between every $5^{\text {th }}$ electrode in a square electrode layout and the measurement pattern replaces every $2^{\text {nd }}$ measurement in the typical method with a measurement between the internal probe and external rings. Note: this figure does not differentiate between upper and lower electrode planes, but all injections and measurements are done between the 2 planes.

\subsection{Results}

Reconstructions of the conductive object with and without additive noise are shown in figure 6.3. With and without additive measurement noise, all models are able to reconstruct the target object. Measurements with internal electrodes appear to reconstruct the target closer to the actual size compared to configurations with external electrodes. 

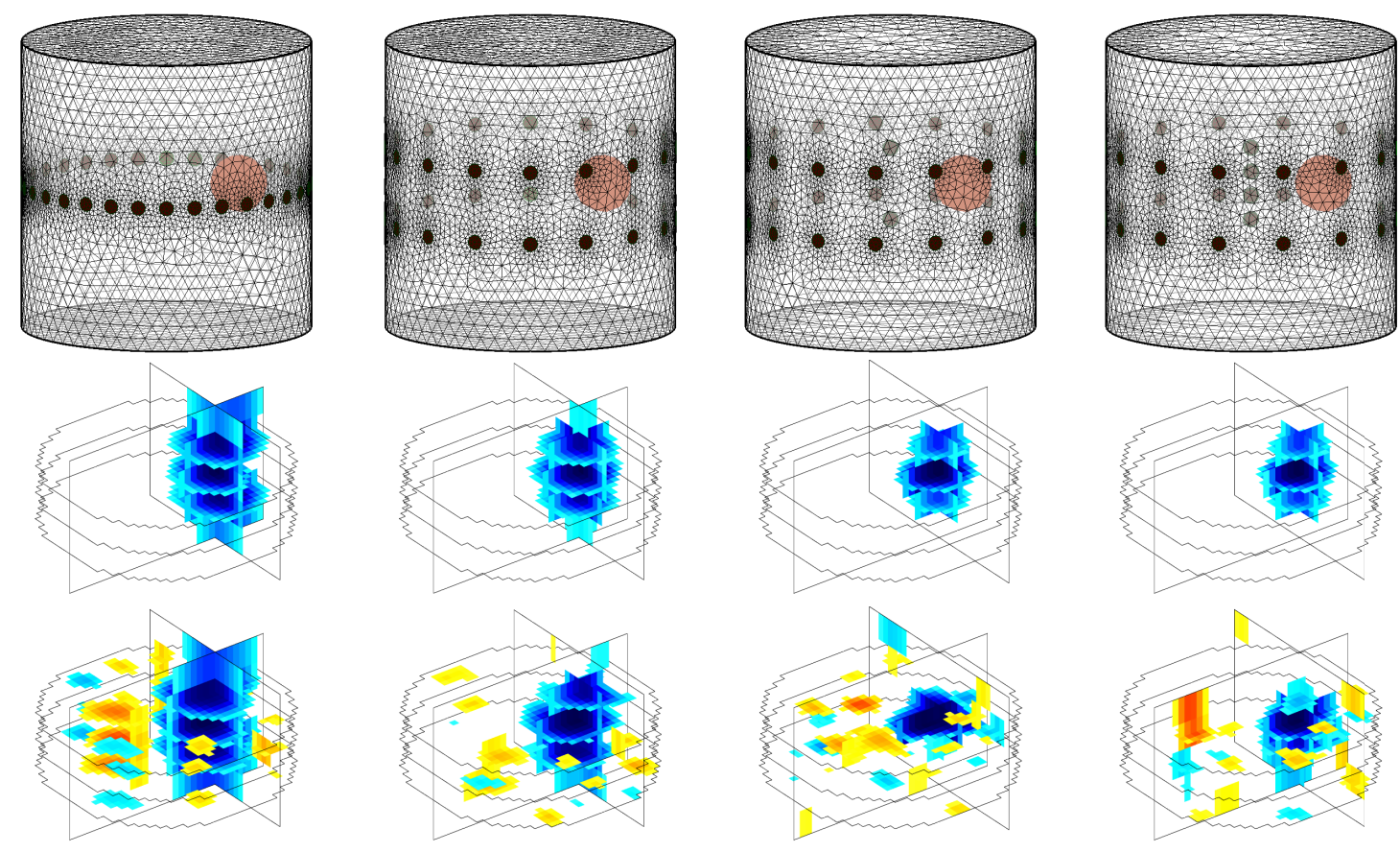

2D

3D(a)

3D(b)

$3 D(c)$

Figure 6.3: The top row shows reconstructions with no additive noise, and the second row shows reconstructions on measurements with $5 \mathrm{~dB}$ of additive noise.

A 2D arrangement of electrodes is not able to distinguish between conductivity changes above and below the electrodes, which contributes to the large reconstructed area. For this reason it is unusual to display reconstructions with a 2D ring of electrodes in 3D, but the resulting image helps to visualize the improvement of $3 \mathrm{D}$ electrode arrangements.

Figure 6.4 shows internal sensitivity distribution changes when using two and four internal electrodes compared to the typical 2D and 3D configurations with only external electrodes. The highest central sensitivity can be seen when using four 
internal electrodes. This electrode configuration also gives a higher sensitivity in between the internal electrodes and the tank boundary.
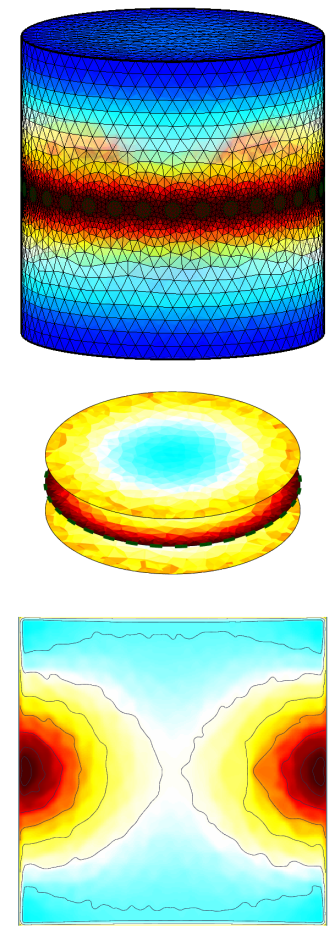

2D
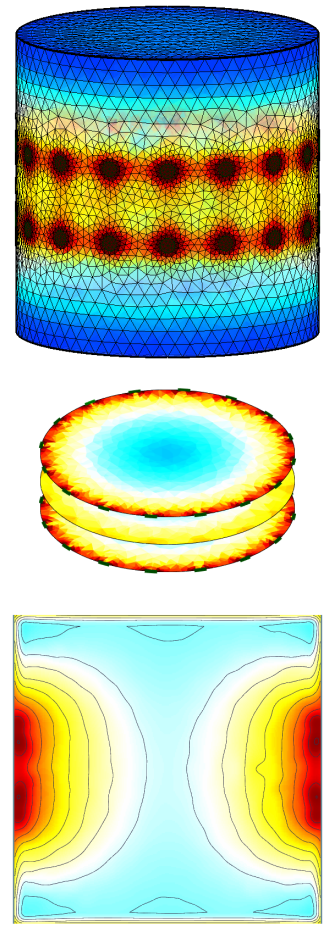

$3 D(a)$
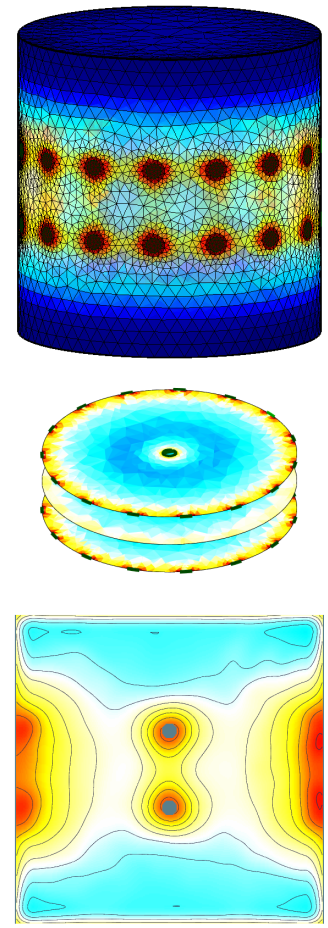

3D(b)
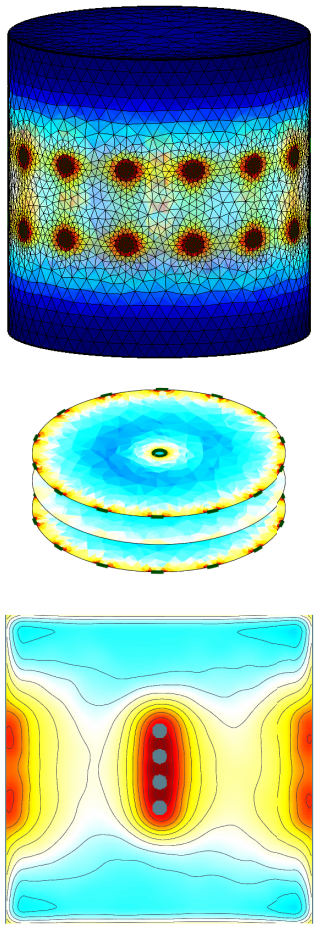

$3 D(c)$

Figure 6.4: Sensitivity distributions for electrode patterns from left to right: A single 2D electrode plane; 2 electrode planes of 16 electrodes each; 2 internal electrodes and 2 external electrode rings of 15 electrodes; 4 internal electrodes arranged between 2 planes of 14 external electrodes

These results show the expected increased sensitivity in the central regions of the model. To further improve internal sensitivity a new measurement pattern is proposed that uses more measurements between the internal probe and peripheral electrodes. The sensitivity of the proposed pattern was compared to the sensitivity profile of the same configuration using the basic "skip 4" injection pattern. Using this 
injection pattern results show a further increase in sensitivity in the internal regions without increasing the measurement acquisition time. The sensitivity distribution for the new injection pattern is pictured in figure 6.5 .
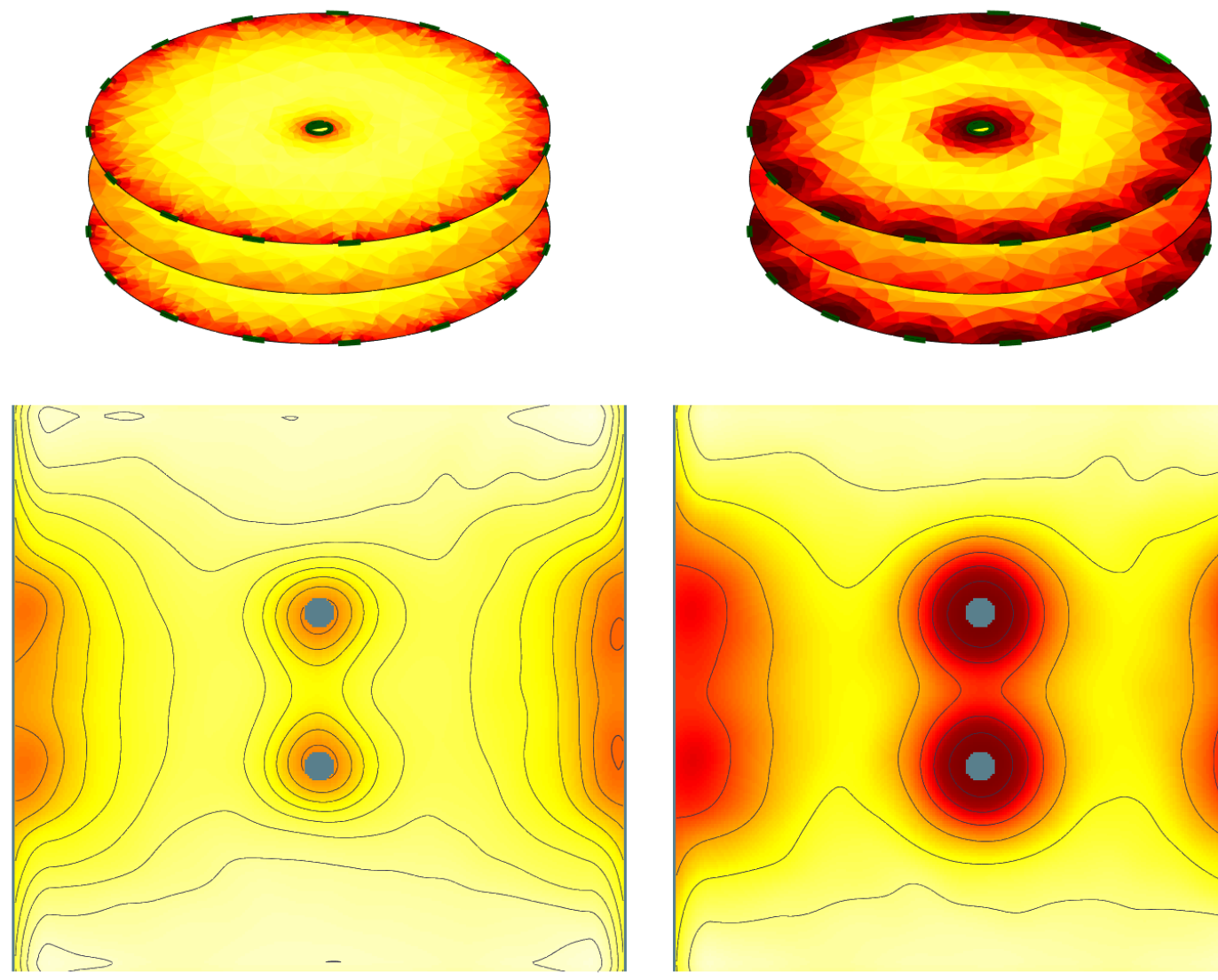

3D(b)

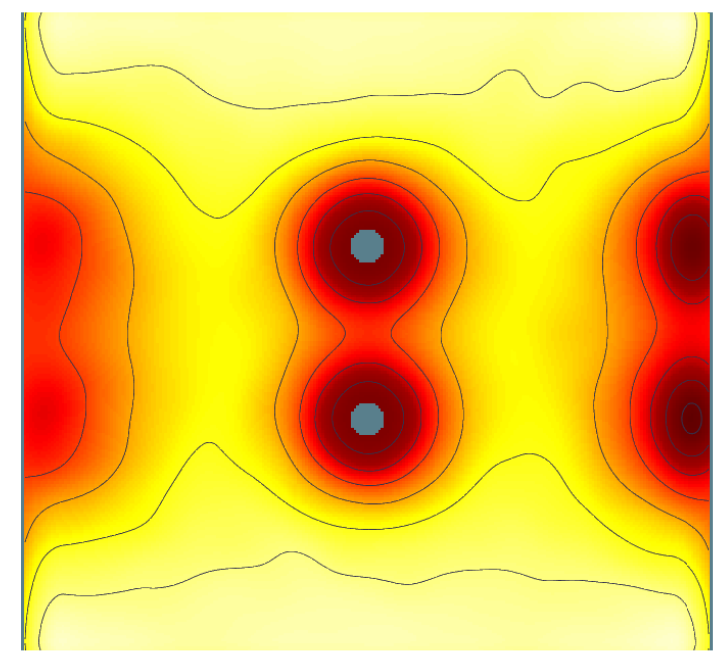

3D(b*)

Figure 6.5: A comparison between the sensitivity distributions for a typical "skip 4" injection pattern pictured on the left $(3 \mathrm{D}(\mathrm{b}))$ and the modified injection and measurement pattern on the right $\left(3 \mathrm{D}\left(\mathrm{b}^{*}\right)\right)$. 


\subsubsection{Sensitivity in an Ovine Model}

A sensitivity comparison was also computed for an ovine model with different electrode configurations and is shown below in figure 6.6. Due to the location of the esophagus in adult sheep and the distance from the heart adding internal electrodes resulted in a minimal sensitivity decrease in the heart region of the model $(<1 \%)$, but there is a large sensitivity increase in the area immediately surrounding the probe.

(A)

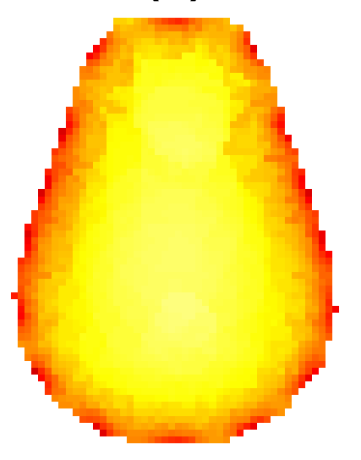

(B)

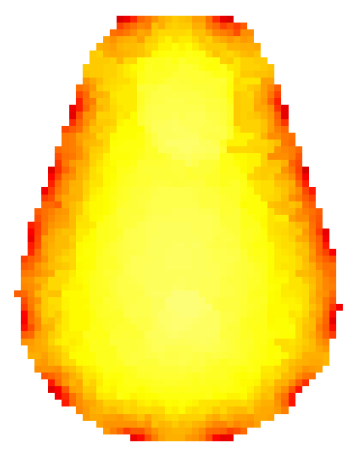

(C)

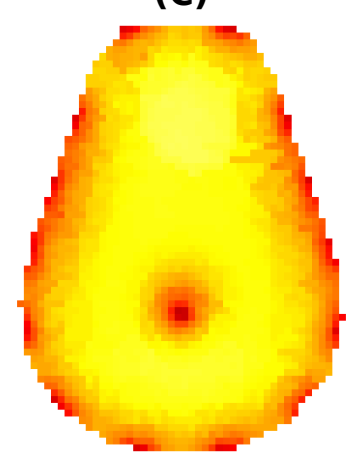

Figure 6.6: Sensitivity distribution averaged across 10 evenly spaced layers between the electrode planes in the lamb model for: A) 32 external electrodes B) 28 external electrodes c) 28 external electrodes and 4 internal electrodes.

\subsection{Discussion}

Based on the simulations presented in this chapter internal electrodes were able to increase the sensitivity in the central regions of the model. Reconstructions in 3D with GREIT were able to accurately reconstruct the location of an object in 3D with both two and four internal electrodes in the presence of $5 \mathrm{~dB}$ measurement noise.

These results align with findings from Nasehi Tehrani et al. (2012) who demon- 
strated that internal electrodes in 2D result in a higher reconstruction accuracy and a much higher internal sensitivity relative to external configurations.

These results demonstrate that the benefits of internal electrodes seen in 2D are also realizable in $3 \mathrm{D}$, but there is more work required to quantify and validate the improvements in real-world settings. Additionally reconstructions on a static target with a static electrode probe are relatively simple. It has been shown that electrode motion and boundary deformation are major contributing factors to error in EIT recordings (Boyle and Adler, 2011; Grychtol et al., 2012), and this has been presented as an additional concern when using internal electrodes that there is currently no solution for (Nguyen et al., 2020). The following chapter explores this source of error in more detail and provides a solution to correct for internal motion.

While the modified current injection pattern increased sensitivity in central regions of the model, it is not likely feasible for real-world use. The majority of commercially available EIT systems use fixed stimulation and measurement spacing and would not be able to accommodate this type of measurement. Additionally if movement of the internal probe is one of the main sources of artefacts when using internal electrodes, it is possible that using the probe for more measurements will not improve the reconstructed image.

Sensitively modelling in an ovine model showed a large increase in sensitivity surrounding the internal probe, but no improvement in sensitivity in the heart region. The ovine model is different than the human model where the heart is much closer to the esophagus. The ovine model may be sufficient to analyze the feasibility of internal electrodes for ventilation and lung perfusion imaging, but may not be comparable to 
data collected in humans due to the difference in physiology.

These results serve as a starting point for 3D EIT, demonstrating that sensitivity increases in the centre of the model when using internal electrodes. Four internal electrodes gave the largest increase in internal sensitivity. The next chapter elaborates on the benefit of internal electrodes demonstrating reconstruction accuracy in simulations.

\subsection{Summary}

Reconstructions using the GREIT algorithm with internal electrodes on an esophageal probe were able to give increased sensitivity in internal regions. This shows promise for increased sensitivity to cardiosynchronous signals and may allow better isolation of pulsatile motion related to perfusion. The following chapter further investigates the impact of motion on internal electrodes. 


\section{Chapter 7}

\section{Internal Electrode Motion}

Preliminary animal reconstructions have been presented in part at: the 21st International Conference on Biomedical Applications of Electrical Impedance Tomography (EIT 2021) (Stowe et al., 2021a).

\subsection{Introduction}

Internal electrodes have been shown several times to improve sensitivity in the internal regions of a model, and improve reconstruction accuracy (Nasehi Tehrani et al., 2012a Nasehi Tehrani et al., 2012b; Nguyen et al., 2020 Pilkington et al., 1989; Schuessler and Bates, 1995). When used in a clinical setting, they have shown an increase in sensitivity to cardiosynchronous EIT signals in most subjects (Czaplik et al., 2014), but they have not been widely used in real-world settings. Image reconstructions using internal electrodes are challenging and have not yet been used consistently in-vivo. One of the main factors that may contribute to confounding 
real-world EIT recordings is movement of the internal probe, although it is not clear how much movement of the probe impacts EIT measurements. Typically when reconstructing EIT images, using difference imaging reduces the impact of systematic errors, but electrodes on an internal probe can move suddenly and may change location during measurements. The effect of this electrode motion is not well understood and currently no analysis exists that explores the effect of internal probe movement on reconstructed images (Nguyen et al., 2012). This chapter presents an analysis of the impact of internal probe movement on EIT reconstructions using internal electrodes, and introduces a technique to correct for probe movement that is up to $10 \%$ of the model radius.

As discussed in section 2.2, several factors contribute towards the cardiosynchronous signal in EIT. One of the largest contributions is movement; both the movement of body structures and the movement of electrodes contribute significantly to the measured impedance and the resulting reconstructed image (Adler et al., 2017b Proença et al., 2015). The previous chapters support that more accurate meshing and adding internal electrodes may help to identify structures and organs, and isolate their impact on reconstructed images, but the problem of electrode motion is amplified when internal electrodes are used. Internal electrodes placed on a probe are challenging to locate within a subject due to variation in individual geometries and incorrectly modelling the electrode locations can introduce an artefact in the image. (Boyle and Adler, 2011). In time difference EIT these effects are reduced (Adler and Boyle, 2017) but the larger problem appears to be the movement of the internal probe relative to the external electrodes between measurements. Recent 
work by Nguyen et al. (2020) reviewed the potential application of internal electrodes in cardio radiofrequency ablation and discussed the need for an analysis of the effect of motion on the internal electrode, and a solution to correct for its impact on EIT reconstructions.

Movement correction algorithms using a movement jacobian have been designed in 2D and 3D (Gómez-Laberge and Adler, 2007; Gómez-Laberge and Adler, 2008, Soleimani et al., 2006) and used to reconstruct electrode movement on 3D models with a 2D arrangement of electrodes (Boyle, 2016). There are several available methods to calculate the movement jacobian (Boyle et al., 2017). The simplest of which, the naïve perturbation method (Gómez-Laberge and Adler, 2008), has been used in this chapter for this proof of concept.

As an internal probe moves towards one side of a model the distance between the probe and the external electrodes changes. This results in lower impedance where the distance has decreased, and a higher impedance where the distance has increased. Without knowing the new probe location we can reduce the effect of this error, but there still remains a mismatch between the modelled and actual probe locations. The resulting artefact that is reconstructed can give hints regarding the motion of electrodes, although it can be difficult to separate the effect of electrode movement from biological impedance changes of interest (Boyle, 2016).

This chapter presents a method that builds on currently available algorithms, to create a new, corrected model that helps to compensate for the effect of electrode movement. The known shape of an artefact due to movement of the probe is used to derive the probe location, and create a corrected model to improve reconstruction 
accuracy in the presence of probe motion.

The goal of this chapter is to develop and validate a technique to reduce the impact of probe movement on image reconstructions, and then to test this technique in an animal model. Although simulations in 2D, and now in 3D, show that internal electrodes can provide a desirable increase in sensitivity, the benefit has not been realized in real-world recordings. The relatively large amount of promising simulation work compared to the limited real-world results suggest that this is a challenging problem. It has been suggested that error due to electrode motion may be a major contributing factor to the EIT signal on internal electrodes (Nguyen et al., 2020), and we believe this may be one of the largest sources of error in real-world EIT measurements. We investigate the impact of motion on internal electrodes and explore techniques to solve this problem. This chapter presents a novel method to correct for electrode displacement artefacts, and evaluates how it is able to improve image reconstruction. This new technique is compared to established techniques for 3D EIT image reconstruction and used to reconstruct ventilation images in an animal model for a limited number of subjects.

\subsection{Methods}

This chapter describes the analysis for both: a simulation study correcting for probe motion in a tank model, and preliminary in-vivo recordings that were conducted in conjunction with a larger experimental protocol. 


\subsubsection{Simulations}

Simulations in a tank model were used to model electrode motion and measure its effect on reconstruction accuracy. Simulations were done using EIDORS (v3.10) (Adler et al., 2017a) and Matlab 2019b (Matworks, Natick, MA, USA). Within EIDORS, meshes were generated using Netgen (version 5.3.1) (Schöberl, 1997).

\subsubsection{Tank Model}

28 external electrodes were placed on a tank model in two planes of 14. 4 internal electrodes were placed evenly between the top and bottom external electrode planes. The tank radius and height were $1 \mathrm{~m}$, and the external electrode radius was $5 \mathrm{~cm}$. The external electrode planes were placed at a height of $0.3 \mathrm{~m}$ and $0.7 \mathrm{~m}$ on the tank. The internal electrodes were spaced evenly between the external electrode planes at $0.3 \mathrm{~m}, 0.433 \mathrm{~m}, 0.567 \mathrm{~m}$ and $0.7 \mathrm{~m}$. The internal electrodes were specified in two different ways. The first used spheres with a radius of $2.5 \mathrm{~cm}$, and the second used a hollow cylinder with a radius of $2.5 \mathrm{~cm}$ containing cylindrical electrodes with a radius of $2.5 \mathrm{~cm}$ and a height of $5 \mathrm{~cm}$. External electrodes were placed in a "square" electrode configuration (Grychtol et al., 2016). Both models are shown in figure 7.1. 
(A)
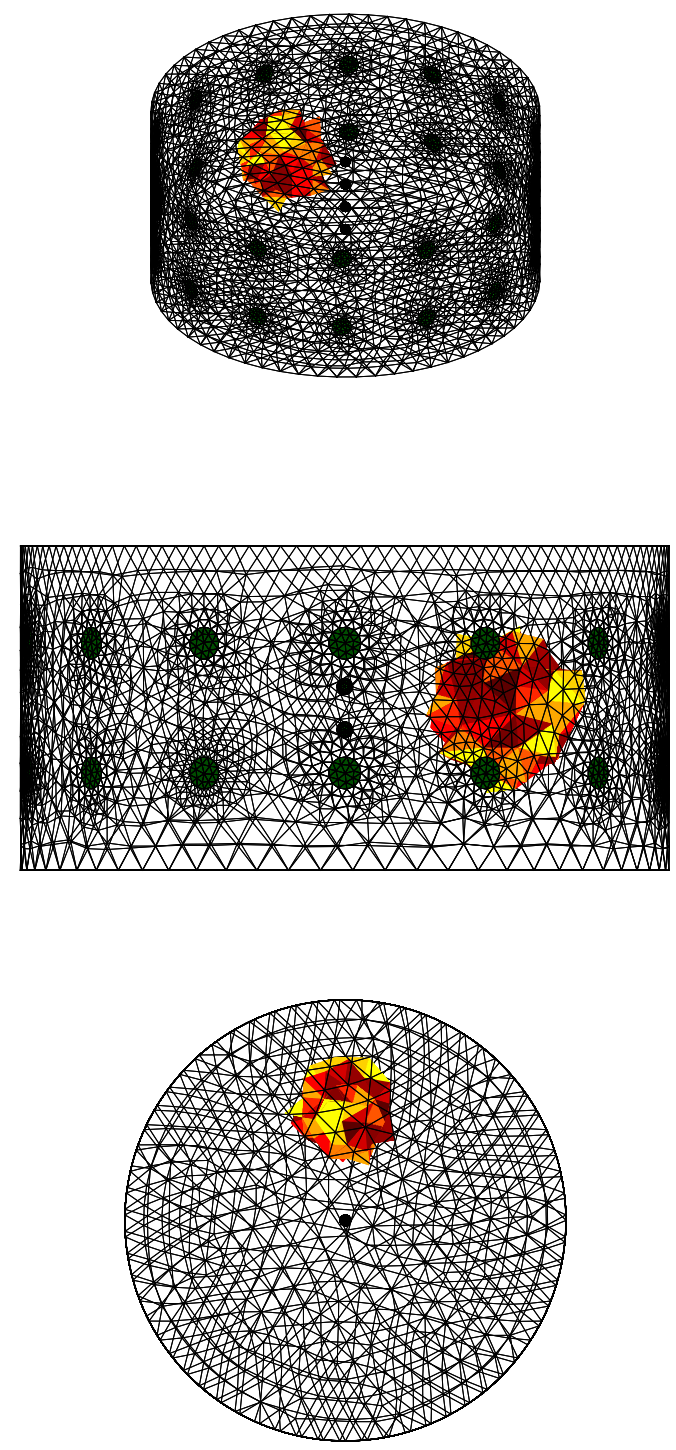

(B)
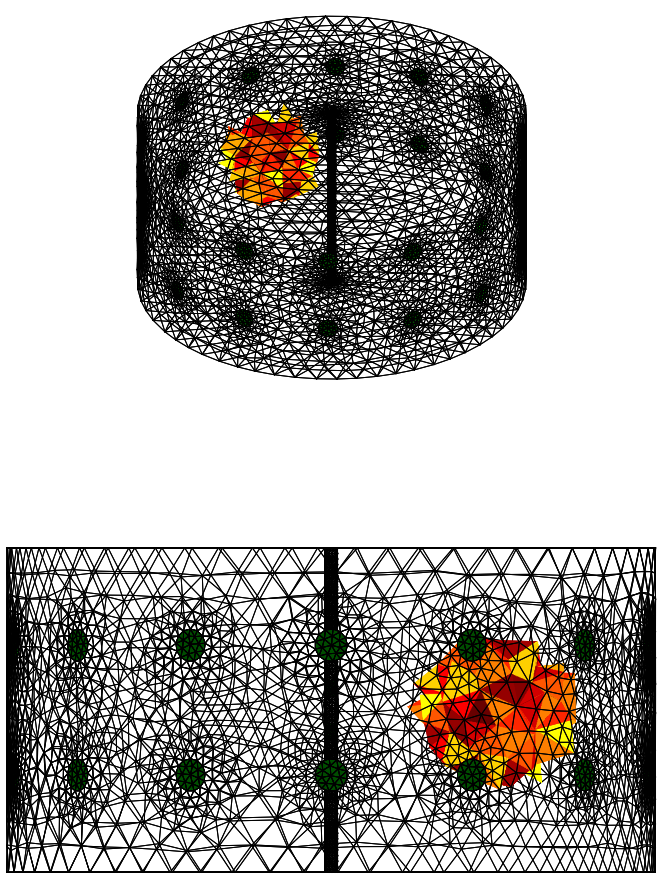

Figure 7.1: $\quad$ On the left (A) internal electrodes were modelled as 4 spherical electrodes between the external electrode planes. On the right (B) the internal electrodes were created using a hole through the centre of the model and using cylindrical electrodes on the inner surface of the model. The conductive region was specified by setting the conductivity of all elements contained in the target to be twice the background conductivity. 
These models were compared to verify that both performed adequately with motion correction, as internal electrodes are challenging to model and many different techniques are used. When using internal electrodes on a complex model, it is currently easiest to add electrodes along a hollow tube cut through the centre, since a small circle can be specified in EIDORS functions that are designed to make extruded models. This circle, when extruded up with the boundary, will form a small hole at the probe location where electrodes can be placed.

To specify a conductive region, elements within a radius were assigned a conductivity of twice the background conductivity of the tank model. The conductive target was placed midway between the centre of the tank and the boundary. The radius of the target was $20 \mathrm{~cm}$. Figure 7.1 shows the conductive target for both internal probe types.

\subsubsection{Measurements}

The skip 4 current injection and measurement pattern was used as it can be easily implemented on most EIT systems, and was shown to have a very even sensitivity distribution in 3D EIT (Grychtol et al., 2016).

Reference measurements were made with no conductive object and electrodes in the centre of the model. Measurements with a conductive object were made with the probe centred, then with the probe was shifted by 1, 5, and 10 percent of the tank radius in a randomized direction. The direction was randomized between trials but was consistent for each of the 1,5 and 10 percent probe error models that were compared. 


\subsubsection{Movement Correction}

As shown in section 2.4.5, the jacobian in typical EIT reconstruction represents the sensitivity of the voltage measurements to a change in conductivity. In this chapter we will refer to this as the conductivity jacobian $\left(J_{C}\right)$. A similar formulation can be used for the movement, but instead of the sensitivity to change in conductivity, we want the jacobian to represent the change in measured voltage sensitivity due to movement of the probe. to calculate the movement jacobian we can use:

$$
\left[J_{M}\right]_{l}=\frac{\Delta \mathbf{V}_{l}}{\Delta \mathbf{r}_{l}},
$$

where $\mathbf{r}$ is the change in position of an electrode $l$. The movement jacobian is a derivative of the measurements with respect to the movement. To approximate the movement derivative we can use a perturbation to shift each electrode on the internal probe by a small amount $(0.001 \mathrm{~m})$, and then calculate the change in voltage measurements (Gómez-Laberge and Adler, 2008).

The movement correction jacobian was calculated using the methods presented by Gómez-Laberge and Adler (2008). In a model with a centred electrode probe and uniform conductivity, each of the four electrodes on the probe was perturbed by 0.001 $\mathrm{m}$ in each of the $\mathbf{x}, \mathbf{y}$, and $\mathbf{z}$ directions. A measurement was made for each electrode and each of the three dimensions of movement. The resulting measurements were divided by the perturbation amount. The measurements on each electrode $\left(V_{j}\right)$ and from each direction of movement $(\mathbf{x}, \mathbf{y}$, and $\mathbf{z})$ were combined to form the movement 
jacobian $\left(J_{M}\right)$ using the following equation from Gómez-Laberge and Adler (2008):

$$
J_{M}=\left[\frac{\partial \mathbf{V}_{j}}{\partial \mathbf{x}}, \frac{\partial \mathbf{V}_{j}}{\partial \mathbf{y}}, \frac{\partial \mathbf{V}_{j}}{\partial \mathbf{z}} \ldots \frac{\partial \mathbf{V}_{n}}{\partial \mathbf{x}}, \frac{\partial \mathbf{V}_{n}}{\partial \mathbf{y}}, \frac{\partial \mathbf{V}_{n}}{\partial \mathbf{z}}\right]
$$

This movement jacobian was used in conjunction with the following single-step formulation for a reconstruction matrix presented by Adler et al. (1994). The regular impedance-based reconstruction matrix is denoted by $R_{C}$ and the jacobian for impedance-based reconstruction is denoted by $J_{C}$.

$$
R_{C}=\left[J_{C}\right]^{T}[W]\left(\left[J_{C}\right][W]\left[J_{C}\right]^{T}+[W]\right)^{-1}
$$

$W$ in the above equation represents the Laplace prior (Soleimani et al., 2006). Combining this formulation with the movement jacobian yields the following equation for the reconstruction matrix $\left(R_{M}\right)$ (Soleimani et al., 2006), where $R_{M}$ denotes the reconstruction matrix for motion correction.

$$
R_{M}=\left[J_{C}\right]^{T}[W]\left(\left[J_{C}\right][W]\left[J_{C}\right]^{T}+\mu\left[J_{M}\right]\left[J_{M}\right]^{T}+[W]\right)^{-1}
$$

In the above equation $\mu$ represents the weighting of the motion correction. In this experiment the motion correction weighting was set to 100 .

Combining equation (7.3) and equation (7.4), a reconstruction matrix to reconstruct exclusively the noise due to movement $\left(R_{N}\right)$ in an image can be generated.

$$
R_{N}=R_{M}-R_{C}
$$


An image $\left(X_{N}\right)$ is reconstructed using the measurements $(b)$ and reconstruction matrix $\left(R_{N}\right)$ as follows:

$$
X_{N}=b R_{N}
$$

Three images: $X_{C}, X_{M}$ and $X_{N}$ were reconstructed from their respective reconstruction matrix.

A new movement correction strategy had been built around the reconstructed image $X_{N}$. As a probe is moved towards electrodes on the edge of the model, conductivity is increased where the distance is decreased resulting in a conductive object in the reconstructed image. Using $R_{N}$ we are able to reconstruct the size and shape of the artefact due to only electrode motion, and determine the approximate direction and amplitude of the probe movement. In the reconstructed image showing only the motion artefact, the centre of mass of the conductive artefact is located. This is assumed to be the direction of the motion. The amplitude of the electrode motion is estimated as half of the distance between the conductive artefact and the probe. This method is illustrated in figure 7.2 Images were reconstructed and displayed on a 64 by 64 grid to give a more accurate representation of the electrode position in the reconstructed image. 
(A)

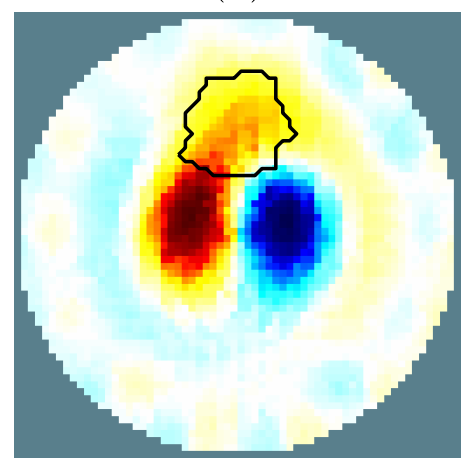

(B)

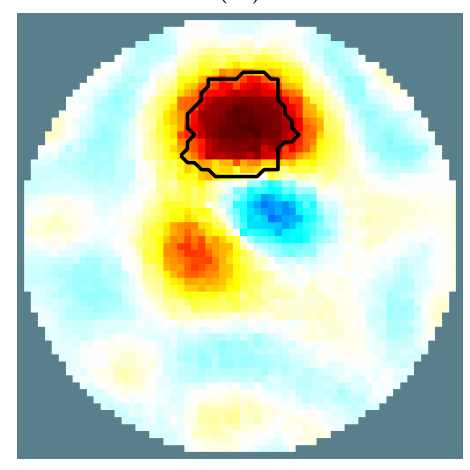

(C)

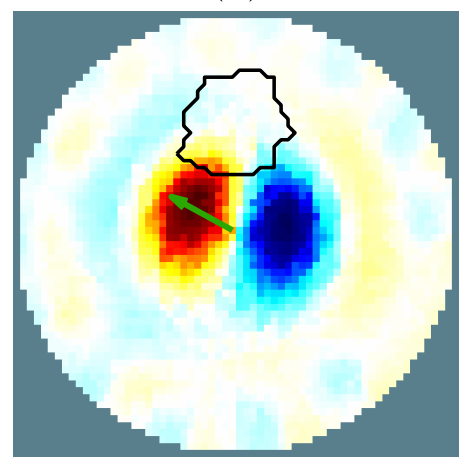

Figure 7.2: This figure shows the 3 scenarios of the initial image reconstruction. Image (A) shows the image reconstructed using the typical conductivity jacobian from equation 7.3 . Image (B) shows the image reconstructed using the movement jacobian and equation equation (7.4). Finally image $(\mathrm{C})$ shows the isolated image artefact reconstructed using the reconstruction matrix in equation 7.5 . The centre of mass of the conductive object in (C) is used to estimate the probe location and direction of movement. The green arrow indicates the calculated direction of movement (multiplied by 5 for visibility). The black line indicates the outline of the true target location. The images depict an average of 10 slices between the external electrode planes.

Using the probe location estimate calculated from $X_{N}$, a new model was reconstructed with the probe repositioned to the identified location. This model was then used to calculate a new reconstruction matrix using equation (7.4) an updated movement jacobian based on the new probe location.

\subsubsection{Image Comparison}

To simplify processing of the reconstructions, 10 images between the external electrode planes were averaged together to generate a 2D representation of the 3D data. This did not allow for analysis of movement in the vertical direction, but we do not expect this to be a major contribution in real-world data. The reconstructed images 
were compared in two ways. First, the accuracy of the reconstruction was evaluated by computing the Jaccard index (Jaccard, 1912) between the actual and imaged boundaries of the conductive target. The imaged boundary was drawn at half the maximum value of the brightest object in the image. The second metric computed was a noise estimate. This was calculated as the amplitude of the imaged object relative to the amplitude of the entire image through the following equation:

$$
N=1-\frac{A_{\text {object }}}{A_{\text {image }}}
$$

Where $A_{\text {object }}$ is the amplitude of the object, $A_{\text {image }}$ is the amplitude of the image, and the ratio is subtracted from 1 so that a noise estimate of 0 corresponds with all of the image signal originating from the conductive target. In this case a low noise estimate is better.

\subsubsection{In-vivo Recordings}

This experiment was approved as an amendment to an existing protocol (2018-2131) by the Université de Sherbrooke Ethics Board. The pregnant ewe carrying two lambs was premedicated using an intramuscular injection of $10 \mathrm{mg} / \mathrm{kg}$ of ketamine and intubated. Anesthesia was maintained using 2-4\% isoflurane in conjunction with conventional mechanical ventilation (CMV). Lambs were birthed prematurely at 120 days of gestation (normal gestation is 147 days) through caesarean section. One lamb was ventilated with either CMV or total liquid ventilation (TLV) for 6 hours. The other lamb served as a paired control to assess lung and cerebral inflammation resulting from ventilation. 
Data were collected in three ewes during CMV using the SenTec EIT Pioneer Set. A skip 4 measurement pattern was used with an injection current of $3 \mathrm{~mA}$ at $30 \mathrm{kHz}$. Electrodes 29 to 32 were connected to the internal electrode probe.

\subsubsection{Internal Electrode Probe Design}

The internal electrode probe consisted of four brass electrodes on a flexible tube. Each electrode was $1 \mathrm{~cm}$ in length and the spacing between electrodes was $2 \mathrm{~cm}$. The electrodes were created from a brass sheet with a thickness of $2 \mathrm{~mm}$ that was bent to the shape of the flexible tubing and soldered to achieve a smooth surface. The electrodes were glued in place on the tube, protruding $2 \mathrm{~mm}$ from probe surface. The tube radius was $0.5 \mathrm{~cm}$. The constructed probe is shown in figure 7.3 . 


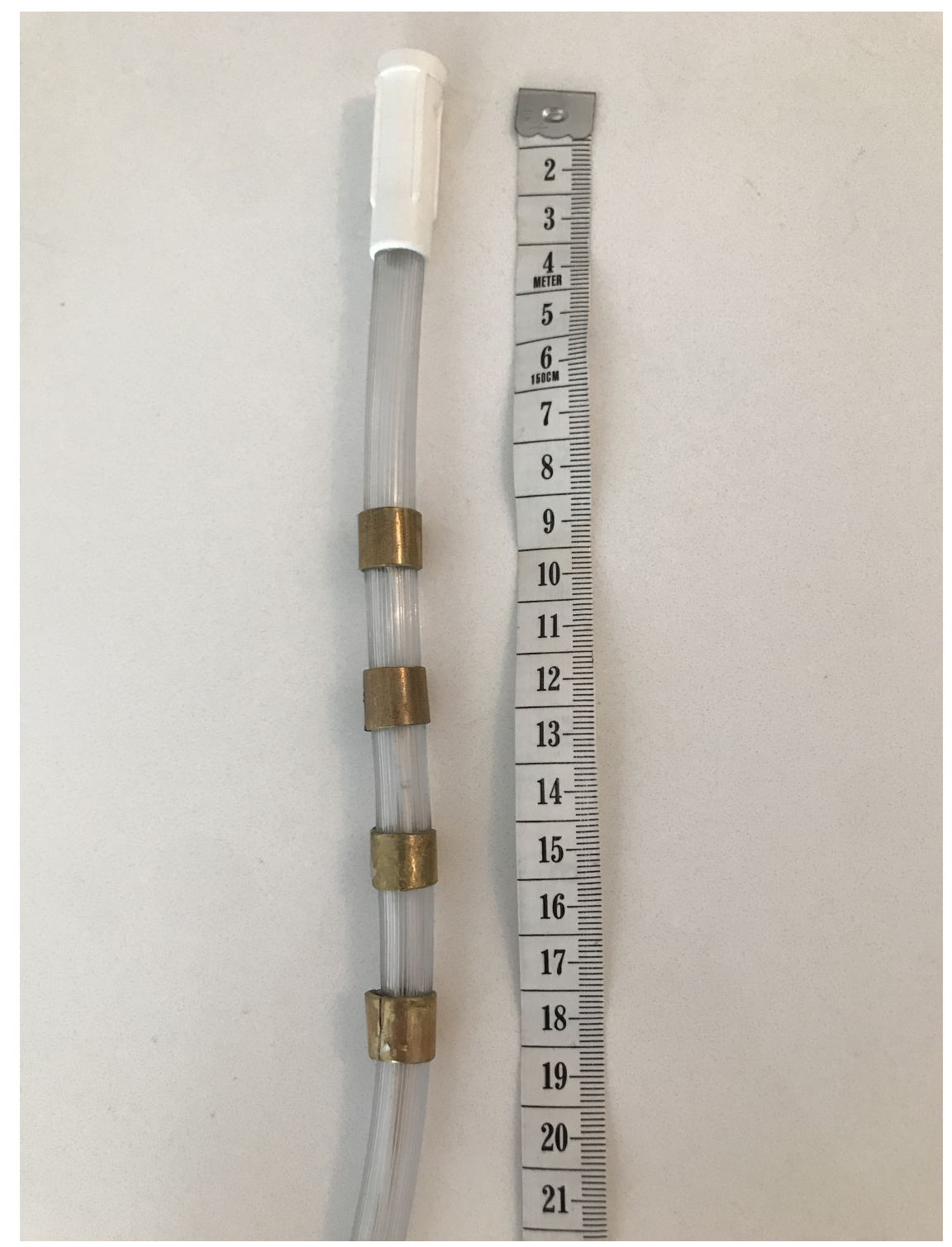

Figure 7.3: A prototype of an internal electrode probe for esophageal use in an ovine model. Four brass electrodes are bent around the outside of a flexible tube designed for esophageal use. Edges of the electrodes are soldered and filed to be smooth. The electrodes are glued in place and wires run down the hollow centre of the tube to connect to the EIT system. 


\subsubsection{Electrode Placement}

The ewes were shaved and 28 silver-silver chloride electrodes were placed in 2 rows immediately behind the front legs with $10 \mathrm{~cm}$ separation and equal spacing around the thorax. The esophageal probe was aligned with the electrodes externally and the required insertion depth was marked. The electrode probe was then coated in a conductive gel for lubrication and to ensure good contact with the esophageal wall, then inserted in the esophagus up to the marked point.

\subsubsection{Ovine Mesh}

An internal probe was added to an ovine mesh by adapting the mk_library_model function in EIDORS (Adler et al., 2017a). A circular region was added to the library geometry, and was extruded upwards to make a hole at the probe location. The probe was inserted into the mesh just anterior to where the spine would be, and posterior to the defined lung region.

The location of the probe in the model relative to lung and heart regions is indicated in figure 7.4 . 


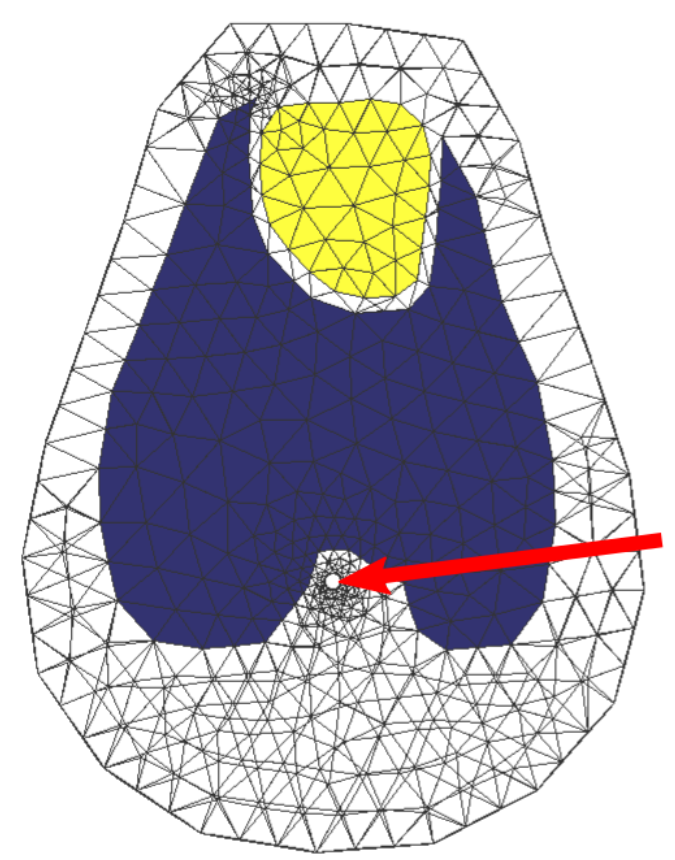

Figure 7.4: This figure shows the probe location relative to the lungs and heart. The probe was located at the expected location of the lungs, just anterior to the spine.

External electrodes were placed in a square pattern on the boundary, and internal electrodes were added as cylindrical objects in the central hole of the model. The resulting model is shown in figure 7.5 . 

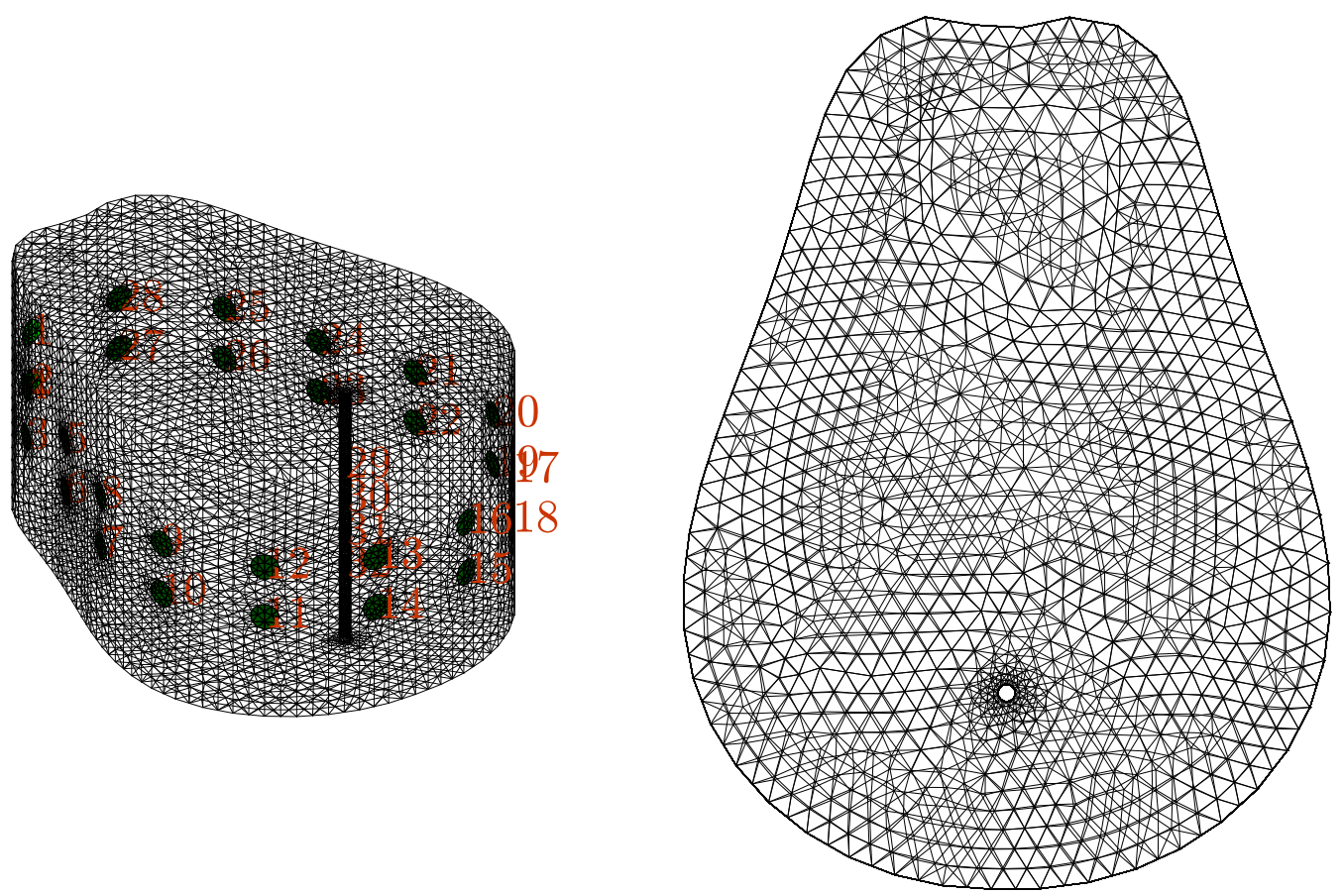

Figure 7.5: An internal electrode was added to an ovine model by creating a hole at the desired probe location and adding cylindrical electrodes at the desired heights. External electrodes were placed in a "square" electrode configuration on the boundary. Electrodes 1 to 28 were external and 29 to 32 were internal.

\subsubsection{Reconstruction}

To reconstruct images 4 techniques were used. First we compared the 3 techniques in section 7.2.2, and GREIT was also used on reconstructions of real data. GREIT for 3D reconstruction has been used with internal electrodes (Nasehi Tehrani et al., 2012a Nasehi Tehrani et al., 2012b), and has been shown to perform well in 3D configurations of external electrodes (Grychtol et al., 2016).

To reconstruct images with GREIT 500 training targets with a size of $5 \mathrm{~cm}$ were specified, and a noise figure of 0.5 was used. Images were reconstructed at 10 
slices between the electrode planes and summed together to form one display image representing the $3 \mathrm{D}$ volume.

\subsubsection{Pulsatile Amplitude}

The goal of this analysis is to quantify the sensitivity increase with regard to per-

fusion. Although the cardiosynchronous signal may have many sources, we use the amplitude of the cardiac frequency in recorded EIT data as a proxy for sensitivity to perfusion-related changes.

To measure the advantage provided by internal electrodes with regard to cardiosynchronous signal detection, a fast Fourier transform (FFT) of each signal was calculated. The cardiosynchronous component was selected as the highest amplitude near the recorded heart rate range of $65-80 \mathrm{bpm}$. This was divided by the amplitude of the ventilation frequency, which was the largest low-frequency amplitude in the FFT of the detrended signal. This was repeated for all 12 recordings across 3 animals.

\subsection{Results}

The following sections present the results for both simulation and in-vivo work.

\subsubsection{Simulation}

When comparing different techniques of modelling internal electrodes, it was found that there was no measurable difference between simulations of models using spherical 
internal electrodes relative to the cylindrical electrodes. The results are presented using the cylindrical model to be consistent with the lamb models. The probe was moved in the $-\mathbf{y}$ direction for figures and calculations in the results.

Figure 7.6 shows the result of the three reconstruction methods. The boundaries of the reconstructed and actual targets are outlined to highlight the performance. For all rows of the figure, the outline of the "true" conductive region is slightly different. This is because when the internal probe is placed in a new location, the entire internal mesh is slightly different. This results in a slight change in the number of elements that are in the target region of the model. All reconstruction techniques were able to reconstruct the object correctly with $1 \%$ movement, but there is more blurring and additive noise in the reconstruction without motion correction. With 1\% movement, both movement correction techniques are able to accurately reconstruct the probe location, but there is a slightly lower level of noise when using the new correction technique. When the probe is moved by $5 \%$, there is a more pronounced difference between reconstruction techniques. When no motion correction is used, the amplitude of the artefact is larger than the amplitude of the reconstructed image. With basic motion correction the conductive object is identified correctly, but there is additional noise present in the image. The new technique reconstructs the image accurately and reduces most of the background noise introduced by probe movement. The new method is the only technique able to reconstruct the correct target location with probe motion of $10 \%$ of the tank boundary between measurements. The Jaccard index and noise estimate for each method are presented below in table 7.1 and table 7.2 
Table 7.1: The Jaccard index was calculated for each of the reconstructions in figure 7.6 Method A does not use any motion correction. Method B incorporates the movement jacobian, and method $\mathrm{C}$ uses the new probe location correction technique. For Jaccard index, a score closer to 1 is better.

\begin{tabular}{cccc}
\hline Movement (\% of radius) & Method A & Method B & Method C \\
\hline 1 & 0.732 & 0.809 & 0.802 \\
5 & 0.038 & 0.654 & 0.808 \\
10 & 0.003 & 0.000 & 0.577 \\
\hline
\end{tabular}

Table 7.2: Noise estimate values calculated for each of the reconstructions in figure 7.6 Method A does not use any motion correction. Method B incorporates the movement jacobian, and method $\mathrm{C}$ uses the new probe location correction technique. For the noise estimates a lower score is better, a score of 0 indicates all image changes occur within the target boundary, and a score less than 1 indicates most of the changes in the image are due to the identified target.

\begin{tabular}{cccc}
\hline Movement (\% of radius) & Method A & Method B & Method C \\
\hline 1 & 0.588 & 0.537 & 0.529 \\
5 & 0.781 & 0.669 & 0.618 \\
10 & 0.777 & 0.747 & 0.718 \\
\hline
\end{tabular}


For movement of $1 \%$ of the tank boundary, motion correction using only the movement jacobian was roughly equivalent to the probe location correction algorithm. Across all other scenarios, the probe location correction algorithm achieved a higher Jaccard score. When interpreting the Jaccard score, a value of 1 indicates a perfect match between the actual and reconstructed boundary and a score of 0 indicates that there is no overlap between the actual and reconstructed locations. Across all scenarios the new technique to correct for motion had the lowest noise estimate score. When other techniques were used additional objects were present in the reconstructions outside of the target objects. When comparing the noise scores, a value closer to 0 is better. A value over 1 indicates that the brightest object in the image was not the actual target. The way that the noise estimate was calculated always assumed the brightest object was the reconstructed image, so no noise scores over 1 were recorded. 
(A)
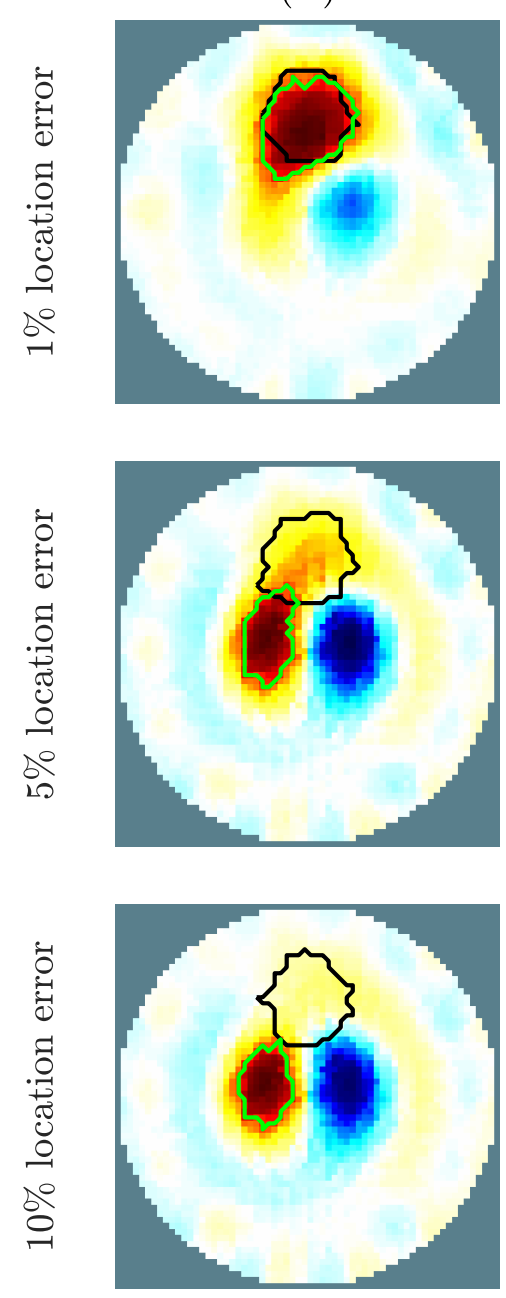

(B)
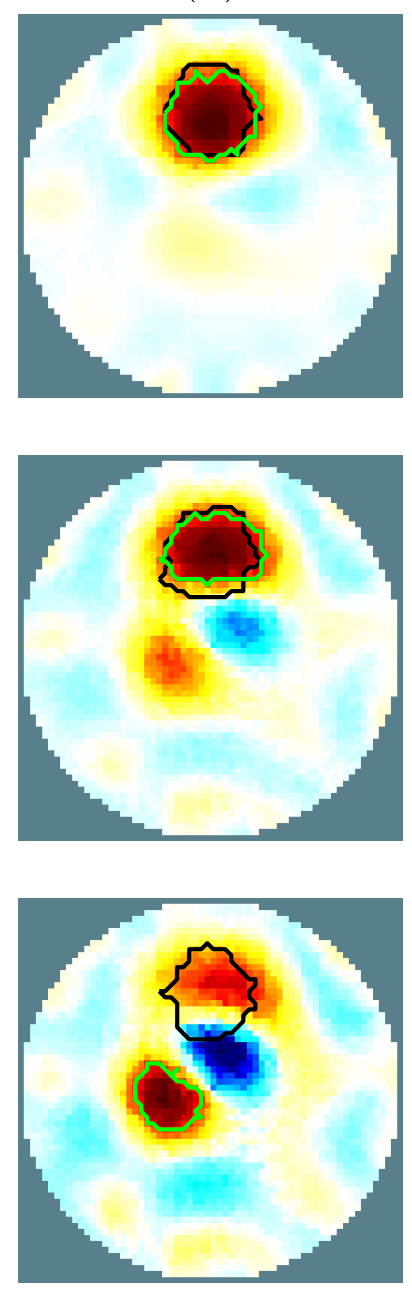

(C)
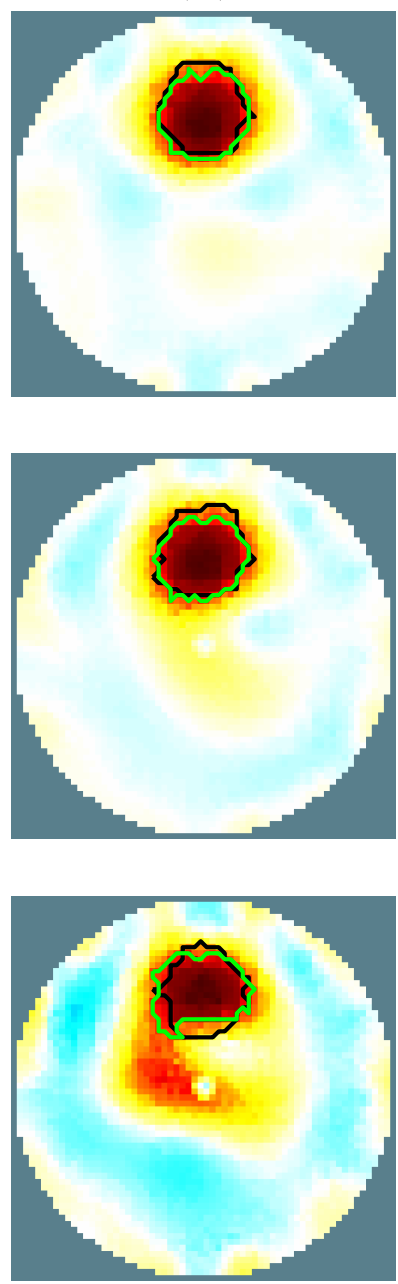

Figure 7.6: The results of the probe location correction are presented. The rows from top to bottom show results with 1,5 , and $10 \%$ shifts in probe location relative to the tank radius. Column (A) shows the results of the reconstruction with no motion correction, column (B) shows the method using the movement jacobian, and column $(\mathrm{C})$ shows the results of the new probe location correction method. The green outline indicates the reconstructed boundary of the conductive target, and the black outline is the actual boundary.

When looking at the reconstructions using the three methods, the new probe correction technique reconstructed the target as the brightest object in all scenar- 
ios. With no motion correction, the target was identified correctly only in the first scenario. Using the movement jacobaian reconstruction method, the target was correctly identified when the probe was moved 1 or $5 \%$ of the tank radius.

\subsubsection{In-vivo}

Recordings in each of the three ewes are presented in this section. Reconstructions with internal electrodes are shown figure 7.7 for a selected breath during a baseline recording in each subject. 
(A)
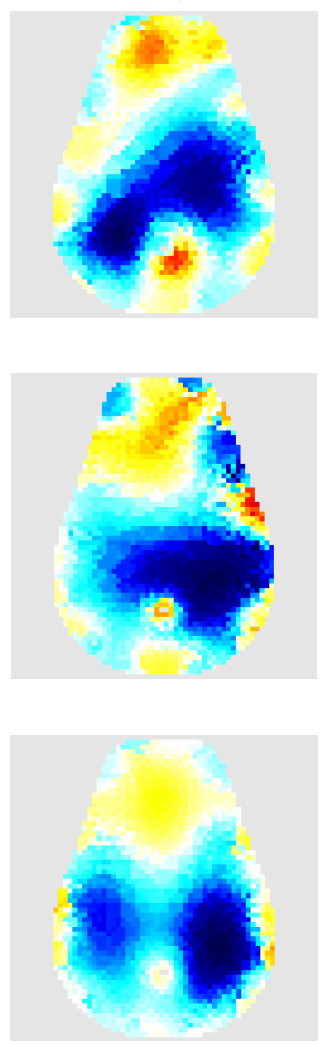

(B)
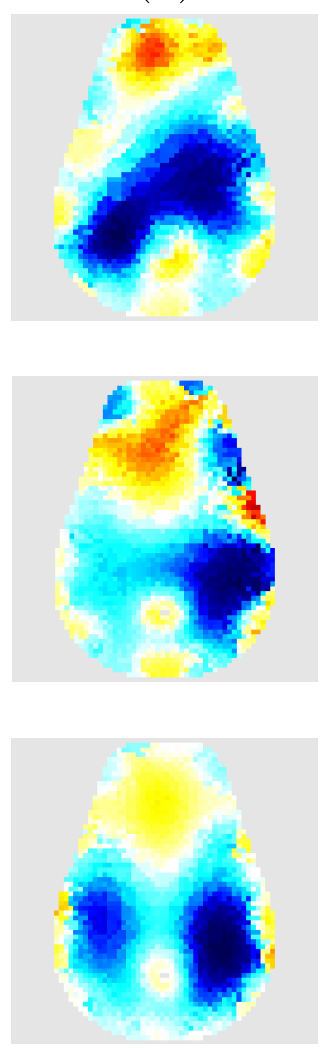

(C)
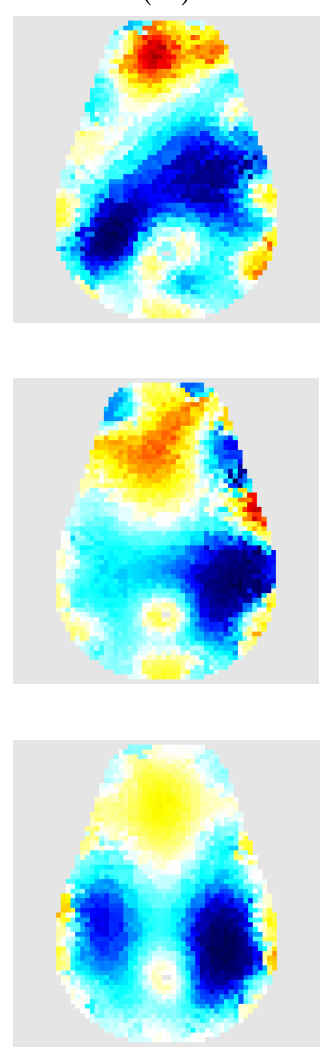

(D)
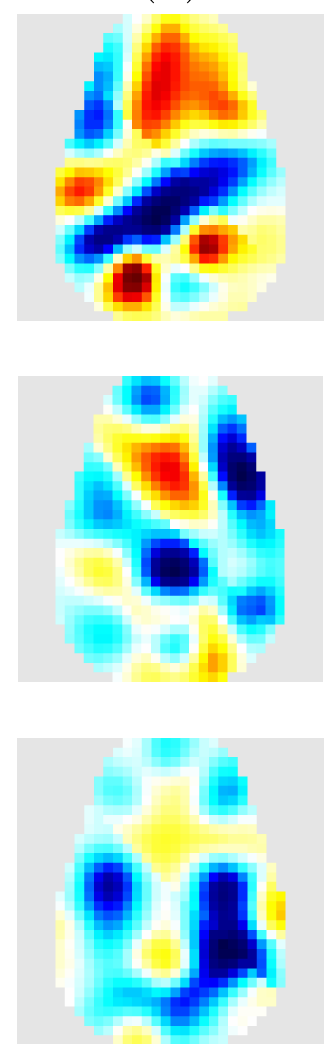

Figure 7.7: Preliminary results on 3 ewes reconstructed from a single, selected breath. Each column represents a reconstruction method. Column (A) uses no motion correction, column (B) uses the movement jacobian and column (C) uses the new method for electrode location correction. Column (D) contains reconstructions using the GREIT 3D algorithm. The number of pixels is different between images due to the methodology of probe localization and the limitations of GREIT.

Reconstructions with movement correction applied to the internal electrodes appear to show a slight improvement in lung distinguishability and a slight reduction in noise surrounding the probe. There is limited difference between the two methods accounting for motion on the electrode.

The ratio of cardiosynchronous to respiratory rate impedance signals is shown 
below in figure 7.8. The results show a higher amplitude of the cardiopulmonary signal relative to ventilation when internal electrodes are used.

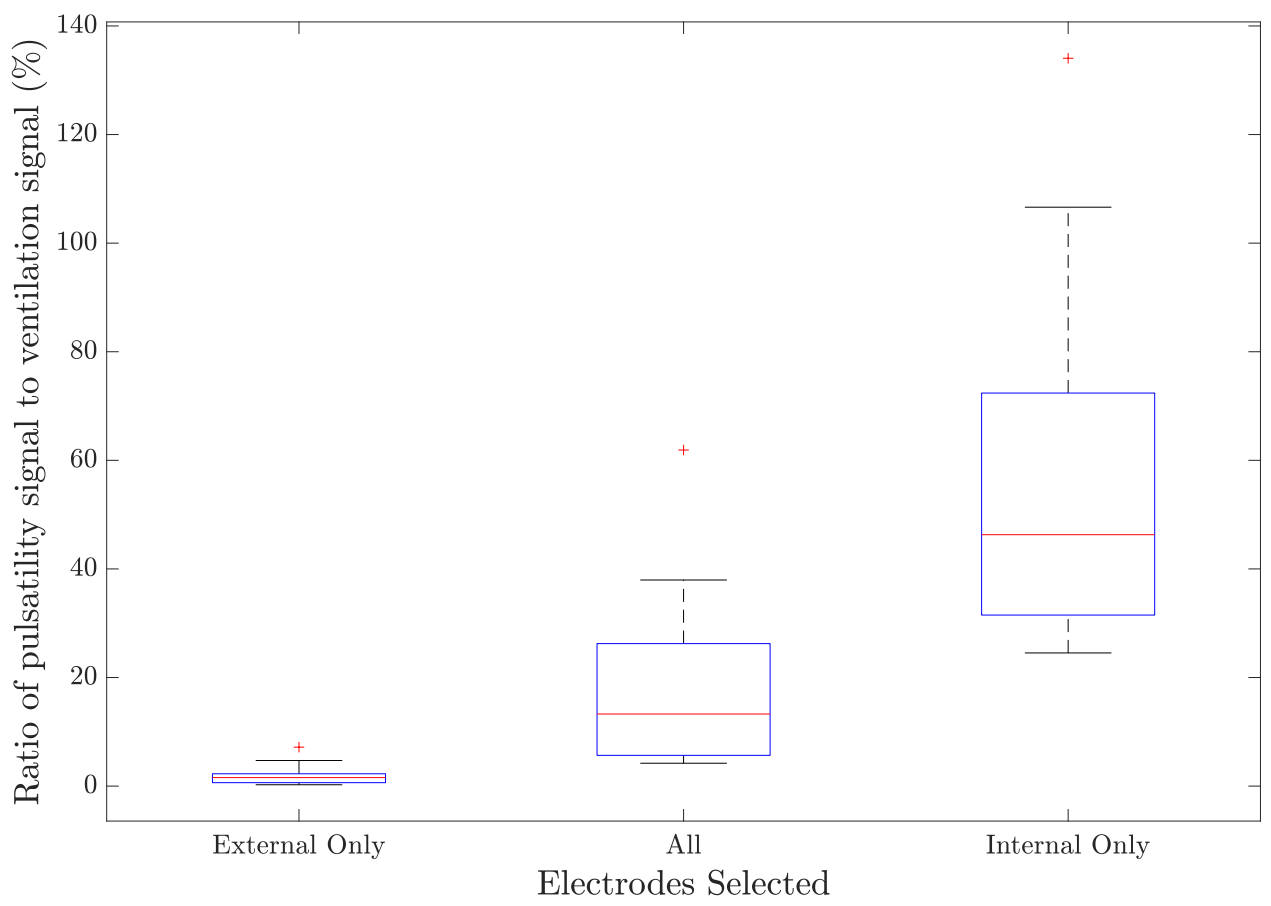

Figure 7.8: The cardiosynchronous signal component was divided by the ventilation frequency component to compare the detectability of the pulsatile changes. The ratio was compared for measurements using only external electrodes, all electrodes, and internal electrodes only.

\subsection{Discussion}

This chapter aimed to provide a technique to reduce noise due to the motion of internal electrodes when reconstructing images in 3D, and to demonstrate the noise reduction technique 
in-vivo. The new technique to reconstruct images with internal electrode motion reconstructed an image of a conductive target more accurately and with less noise in the surrounding image than currently available reconstruction techniques. Invivo reconstructions showed a slight improvement in lung distinguishability when correcting for motion artefacts on internal electrodes compared to methods without this correction including GREIT. Results in 3D also aligned with results from a 2D study in a porcine model showing an increased cardiosynchronous component on the internal electrode measurements (Czaplik et al., 2014).

It was found that GREIT performed poorly when reconstructing data with internal electrodes. It is thought this is because the figures of merit for GREIT were designed and optimized to work with external electrodes where sensitivity is low in the centre of the model and high at the edges (Adler et al., 2009). An adaptation to GREIT that is able to account for internal electrodes and motion of an internal probe is planned as a continuation of this project.

Two types of internal electrodes were modelled in this chapter. One with spherical internal electrodes and one with a hollow probe and cylindrical electrodes. Both meshing and creating internal electrodes can be challenging, due to limited meshing tools for using internal electrodes with EIT. The method of adding internal probes to anatomical models using a hollow region in the centre of the model was created for this project. Code to generate this model with internal electrodes is planned to be included in a future release of EIDORS.

The noise estimate score calculations were not an ideal representation of the noise in the images due to the way the reconstructed object was identified. Since the 
brightest object was always identified as reconstructed the object, the noise estimate was artificially low in some cases. Comparing the amplitude of changes contained within the true object boundaries could be a better metric of the noise present in the image. However, this would also be dependent on the accuracy of reconstruction and would have favoured the method that reconstructed the object most accurately. The boundary of the true target also changes due to differences in element locations as the probe is moved, so the comparison would not be consistent across all probe movement states. Despite this limitation, the noise estimate does help to quantify noise seen in the images for each technique. The new method performed better than existing techniques across all situations according to the computed noise metrics.

In simulated cases of less than $1 \%$ error in probe location, very little difference in reconstruction accuracy between the two motion correction algorithms was detected. Despite this, a slight reduction in background noise in the image was observed.

Despite the improvements to reconstruction accuracy and noise reduction using the new method, there are several limitations to its use. The largest is the increased modelling time required. This method required an extra model, and inverse solution compared to the single step solutions. For simple models such as a tank the added time difference is small, but for complex models this could increase reconstruction time drastically. When several frames are reconstructed, each requires a unique model with a modified probe location, increasing the image reconstruction time. To reduce the reconstruction time, correcting the model only when the probe location error is greater than $1 \%$ of the model radius may be helpful. At this level of movement, the benefits of the probe localization technique are not substantial. 
Additionally, in the images presented probe movement did not appear to be large. It is not yet clear what the typical movement of the electrode probe is expected to be in the esophagus. To verify the position in an animal model future trials could use an accelerometer in the electrode probe to give a measure of probe movement and determine the accuracy of the probe position calculation in real-world applications.

When using internal electrodes to image blood flow, the perfusion of the esophagus itself could contribute to the overall signal measured. Previous research in cats has shown that the esophageal perfusion is very small relative to other organs in the abdomen (Hollwarth et al., 1986) and we do not expect a large contribution to the perfusion signal from the esophagus.

Previous research has also reconstructed for motion direction directly using the movement jacobian (Boyle, 2016, Gómez-Laberge and Adler, 2008; Soleimani et al., 2006). This technique for electrode estimation has not been implemented using internal electrodes for this research, but a comparison between the two electrode position estimation techniques is required for complete validation of the demonstrated method.

Previous studies with internal electrodes showed cases where contact impedance of the probe was inadequate for electrodes placed on the breathing and feeding tubes (Czaplik et al., 2014). The electrodes used were shown to have a good contact impedance on the SenTec Pioneer Set interface, but the current design protrudes slightly from the tube and may be challenging to integrate into a clinical esophageal or tracheal tube. Additionally, given that in a clinical setting current injection in the thorax may present safety concerns, it is also important to quantify the sensitivity 
increase when the internal probe is used for voltage measurements only. Future simulations will focus not only on assessing the safety of internal electrodes, but also evaluating the utility of internal electrodes if no internal current injections are possible.

Sanchez et al. (2013) used an array of internal electrodes in the lung during biopsy procedures, and suggested that the increased proximity to the tissue could allow more physiological information to be obtained. A more complete evaluation is required to measure the benefits of internal electrodes in 3D compared to typical external configurations. The previously seen lung distinguishability and larger cardiosynchronous signal amplitude found in 2D (Czaplik et al., 2014) have been repeated with this $3 \mathrm{D}$ configuration, but it is still unclear to what degree these signal changes may be useful.

Increased lung separability may be useful for determining the difference between right and left lung ventilation, which can be used to quantify ventilation performance (Sage et al., 2018) and is an important step in detecting ventilation and perfusion mismatch (Kircher et al., 2021; Leonhardt and Lachmann, 2012; Stowe et al., 2019). Increased sensitivity to pulsatile flow may also help to improve measures of cardiopulmonary activity where detecting pulsatile flow and motion in specific regions of a model is desired (Braun et al., 2018a; Proença et al., 2020). 


\subsection{Summary}

Probe displacement introduces large errors when using reconstruction algorithms that do not account for movement. This chapter presents a new technique designed to reduce the effect of probe motion. This new method was found to reconstruct a target object more accurately and with less background noise in a tank model compared to existing methods. Internal electrodes in conjunction with a 3D external configuration were also used in-vivo to measure ventilation. Images of breaths across three subjects showed an improvement in lung distinguishability when correcting for motion, and the average ratio of cardiosynchronous to pulmonary signal was increased when using internal electrodes. 


\section{Chapter 8}

\section{Conclusion}

This thesis investigated mesh refinement and internal electrodes as tools to advance perfusion imaging. EIT has very low sensitivity in the centre of the thorax where majority of the perfusion changes of interest occur. The source of the cardiosynchronous EIT signal is also poorly understood, and many factors may contribute to the measured perfusion signal. This thesis presents several contributions to the field of perfusion imaging in EIT. We investigated the limitations of current EIT perfusion imaging and compared the difference between measures of perfusion using a contrast agent and those relying on the cardiosynchronous signal. A technique to control mesh refinement was developed to create meshes with adequate sensitivity accuracy throughout an entire model. This technique can also guide refinement strategies in high sensitivity regions surrounding internal electrodes. A 3D EIT configuration is presented with four internal electrodes that gives high sensitivity in the centre of the

model, and accurately reconstructs a conductive target in the presence of Gaussian 
measurement noise. Finally, we present a method to calculate movement of an internal probe and correct for positioning error in a model. Electrode motion can add artefacts to images that overpower the component of interest, and add background noise to the reconstructed images. This technique uses characteristic information in the reconstructed image related to probe movement to estimate the actual probe location and create a corrected model. The corrected model reduced the effect of movement on reconstructed images.

\subsection{Summary of Findings}

Chapter 3 compared several EIT measures of perfusion to investigate the potential of EIT as a device to monitor perfusion. Despite the different signal origins, both filtering-, and bolus-based techniques resulted in similar perfusion estimates. We found that perfusion estimation with EIT is viable, and hypothesize that improved sensitivity and reconstruction accuracy could improve perfusion measures and clinical viability.

In chapter 4 we explore meshing techniques to reduce error when calculating sensitivity, and ensure mesh density is adequate around electrodes. When mesh density dissipates evenly away from electrodes, we recommend the balance point of the nodes in a mesh should be towards the electrodes. A balance point at $80 \%$ of the distance between the lowest sensitivity region of a model and the electrodes minimized error when calculating sensitivity. This recommendation can be used to generate meshes that are highly accurate when calculating the sensitivity, with fewer 
elements than meshes that are uniformly refined.

In chapter 5 a tool to generate accurate, custom meshes from CT images was presented. This tool automatically segmented the lung and external boundaries from CT images, and allowed manual verification of the segmented boundaries. The segmented boundaries were used to generate custom EIT meshes. Ventilation images on a small number of subjects showed a small improvement over generic models when measuring the centre of mass of the ventilated region. This tool was used to create meshes with individualized, accurate boundaries for patients with CT data.

Chapter 6 presented a sensitivity analysis and simulated reconstruction accuracy of novel electrode configurations with internal electrodes. These simulations show an increased sensitivity in internal regions when internal electrodes are used. Reconstructions of a conductive object also show that internal electrodes can be used to reconstruct the location of a conductive target in the presence of noise. From this work we determined that internal electrodes could be used in 3D to image conductivity changes within the body and could improve sensitivity to internal conductivity changes.

In chapter 7 we analyze the effect of internal electrode motion on reconstructed images. Moving the probe only $1 \%$ of the model radius added artefacts to images reconstructed without motion correction strategies. Available motion correction techniques reduced the impact of electrode movement up to $5 \%$ of the tank boundary, but still showed an increase in image noise. We present a technique to isolate motion artefacts and use this to estimate the true probe location. A new model with a corrected probe location was then used to reconstruct images. Images reconstructed 
with the new technique accurately located a conductive object when the probe moved up to $10 \%$ of the model radius between measurements, and reduced background noise in all probe movement scenarios.

\subsection{Future Work}

There are several avenues that we recommend be explored further from the work and projects presented in this thesis.

- Work is currently underway to test the automatic segmentation tool on a wider number of subjects. We are currently working with the Peking Union Medical College in Beijing, China to obtain more CT and EIT data from ARDS patients.

- The effect of electrode placement errors on reconstruction accuracy when creating custom meshes from CT images is unclear. Incorrectly modelled electrodes degrade reconstruction accuracy (Boyle and Adler, 2011), and incorporating the correct electrode location into the custom meshes could yield more accurate EIT images. We discuss some possibilities in chapter 5 to identify the correct electrode locations, including using photographs to identify electrode locations and estimating electrode placement from the movement jacobian. Chest expansion can have a large effect on EIT images (Adler et al., 1994), and may impact the accuracy of a custom model as the patient moves. Correctly modelling the impact of electrode and boundary movement during breathing could help to further improve reconstruction accuracy. 
- When creating meshes from CT images, errors often occur when electrode placement does not work well on the irregular boundary. Some existing techniques allow electrode placement on arbitrary mesh surfaces (Grychtol and Adler, 2013), but these techniques do not natively offer features for advanced mesh refinement control or use with internal structures. Therefore, future work could focus on developing a method to facilitate meshing and electrode placement on arbitrary boundary shapes.

- Further work is required to determine the safety requirements for internal electrodes in human use. The IEC guidelines (International Electrotechnical Commission, 2021) have strict requirements for the injection of electrical current near the heart, and the current density on internal electrodes should be examined to ensure that it does not exceed current safety standards. It is also possible to use internal electrodes without injecting currents internally, but this could reduce the sensitivity benefits found when using internal electrodes. Further investigation could determine if there are benefits to using internal electrodes only for recording voltage measurements.

- Cardiovascular disease has also been shown to impact the flexibility of vasculature (Glasser et al., 1997) and may reduce the volume changes that occur within the cardiovascular system as the heart beats. It is not currently clear to what degree EIT images of perfusion depend on the flexibility of blood vessels. Future work should examine the ability of EIT to image and detect perfusion in patients with cardiovascular disease. Using an internal electrode arrangement could help to detect the reduced volume changes in sick patients and improve 
the ability of EIT to work in these challenging circumstances. Evaluating the usability of internal electrodes in sick patients is vital since the primary use case is in critically ill patients that require esophageal or tracheal tubes.

- When reconstructing images using internal electrodes with GREIT, internal electrode motion caused artefacts in the image. It is not clear which aspect of the electrode motion introduces extra artefacts in GREIT. Work is planned to create an addition to GREIT that will improve reconstruction with internal electrodes and correct for internal probe movement.

- It has been suggested that the contact impedance of internal electrodes should be matched to the external electrodes (Nasehi Tehrani et al., 2012b), but it is not clear to what degree differences in contact impedance and size impact the reconstruction. An investigation into the ideal size, shape and electrical properties of internal electrode could help to inform their use in a clinical setting.

- The novel technique introduced to correct for probe location uses reconstructed images to determine the position of the internal probe. Further investigation is required to determine if the electrode position can be calculated directly from the reconstruction matrix. Previous work has shown that the movement direction of individual electrodes can be calculated directly from the reconstruction matrix (Soleimani et al., 2006), but this technique does not reconstruct for position. A comparison between techniques to compare the accuracy of each could help to estimate the probe location more accurately. 


\subsection{Conclusion}

This thesis presents tools to analyze and control mesh refinement, generate custom meshes of arbitrary geometry, and correct for movement artefacts when reconstructing images using internal electrodes. This investigation revealed that customized meshes can increase the accuracy with which ventilation is reconstructed, and that internal electrodes can be used to increase sensitivity in the centre of a subject and reconstruct images in an animal model. This project contributes to increasing internal sensitivity of EIT measurements and demonstrates that refined meshes and internal electrodes may improve measures of perfusion and help to make EIT a viable tool for continuous perfusion monitoring at the bedside. 


\section{Bibliography}

Abe, T., Madotto, F., Pham, T., Nagata, I., Uchida, M., Tamiya, N., Kurahashi, K., Bellani, G., \& Laffey, J. G. (2018). Epidemiology and patterns of tracheostomy practice in patients with acute respiratory distress syndrome in ICUs across 50 countries. Critical Care, 22(1).

Adler, A., Boyle, A., Crabb, M. G., Grychtol, B. L., Lionheart, W. R. B., Tregidgo, H. F. J., \& Yerworth, R. (2017a). EIDORS version 3.9. Proc. 18th Int. Conf. on Biomed. Applications of EIT.

Adler, A., \& Guardo, R. (1996). Electrical impedance tomography: Regularized imaging and contrast detection. IEEE Transactions on Medical Imaging, 15(2), $170-179$.

Adler, A., Guardo, R., \& Berthiaume, Y. (1994). Impedance imaging of lung ventilation: Do we need to account for chest expansion? Proceedings of 16th Annual International Conference of the IEEE Engineering in Medicine and Biology Society, 1, 534-535 vol.1.

Adler, A., Amato, M. B., Arnold, J. H., Bayford, R., Bodenstein, M., Böhm, S. H., Brown, B. H., Frerichs, I., Stenqvist, O., Weiler, N., \& Wolf, G. K. (2012). 
Whither lung EIT: Where are we, where do we want to go and what do we need to get there? Physiological Measurement, 33(5), 679-694.

Adler, A., Arnold, J. H., Bayford, R., Borsic, A., Brown, B., Dixon, P., Faes, T. J. C., Frerichs, I., Gagnon, H., Gärber, Y., Grychtol, B., Hahn, G., Lionheart, W. R. B., Malik, A., Patterson, R. P., Stocks, J., Tizzard, A., Weiler, N., \& Wolf, G. K. (2009). GREIT: A unified approach to 2d linear EIT reconstruction of lung images. Physiological Measurement, 30(6), S35-S55.

Adler, A., \& Boyle, A. (2017). Electrical impedance tomography: Tissue properties to image measures. IEEE Transactions on Biomedical Engineering, 64(11), $2494-2504$.

Adler, A., Grychtol, B., \& Bayford, R. (2015). Why is EIT so hard, and what are we doing about it? Physiological Measurement, 36(6), 1067-1073.

Adler, A., \& Lionheart, W. R. B. (2006). Uses and abuses of EIDORS: An extensible software base for EIT. Physiological Measurement, 27(5), S25-S42.

Adler, A., Proença, M., Braun, F., \& Solà, J. (2017b). Origins of cardiosynchronous signals in EIT. EIT $201 \%$.

Aljizeeri, A., Cocker, M. S., \& Chow, B. J. W. (2013). CT vs SPECT: CT is the first-line test for the diagnosis and prognosis of stable coronary artery disease. Journal of Nuclear Cardiology, 20(3), 465-472.

Allaud, L., \& Martin, M. (1977). Schlumberger: The history of a technique. Wiley.

Aristovich, K. Y., Santos, G. S. d., Packham, B. C., \& Holder, D. S. (2014). A method for reconstructing tomographic images of evoked neural activity with 
electrical impedance tomography using intracranial planar arrays. Physiological Measurement, 35(6), 1095-1109.

Ashbaugh, D. G., Bigelow, D. B., Petty, T. L., \& Levine, B. E. (1967). Acute respiratory distress in adults. Lancet (London, England), 2(7511), 319-323.

Axel, L. (1980). Cerebral blood flow determination by rapid-sequence computed tomography: Theoretical analysis. Radiology, 137(3), 679-686.

Barber, D. C., \& Brown, B. H. (1984). Applied potential tomography. Journal of Physics E: Scientific Instruments, 17(9), 723-733.

Barker, R., Ashby, E. L., Wellington, D., Barrow, V. M., Palmer, J. C., Kehoe, P. G., Esiri, M. M., \& Love, S. (2014). Pathophysiology of white matter perfusion in alzheimer's disease and vascular dementia. Brain, 137(5), 1524-1532.

Bartocci, M., Serra, G., Basano, L., Canepa, F., \& Ottonello, P. (1999). Cerebral blood-flow monitor for use in neonatal intensive care units. Computer Methods and Programs in Biomedicine, 59(1), 61-73.

Bates, J. H. T., \& Smith, B. J. (2018). Ventilator-induced lung injury and lung mechanics. Annals of Translational Medicine, 6(19), 378.

Bhuyan, U., Peters, A. M., Gordon, I., Davies, H., \& Helms, P. (1989). Effects of posture on the distribution of pulmonary ventilation and perfusion in children and adults. Thorax, 44(6), 480-484.

Borges, J. B., Suarez-Sipmann, F., Bohm, S. H., Tusman, G., Melo, A., Maripuu, E., Sandström, M., Park, M., Costa, E. L. V., Hedenstierna, G., \& Amato, M. (2012). Regional lung perfusion estimated by electrical impedance tomogra- 
phy in a piglet model of lung collapse. Journal of Applied Physiology, 112(1), $225-236$.

Boyle, A., Crabb, M. G., Jehl, M., Lionheart, W. R. B., \& Adler, A. (2017). Methods for calculating the electrode position jacobian for impedance imaging. Physiological Measurement, 38(3), 555-574.

Boyle, A. (2016). Geophysical applications of electrical impedance tomography (Doctoral dissertation). Carleton University. Ottawa, Canada.

Boyle, A., \& Adler, A. (2011). The impact of electrode area, contact impedance and boundary shape on EIT images. Physiological Measurement, 32(7), 745-754.

Braun, F., Proença, M., Adler, A., Riedel, T., Thiran, J.-P., \& Solà, J. (2018a). Accuracy and reliability of noninvasive stroke volume monitoring via ECGgated 3d electrical impedance tomography in healthy volunteers. PLoS One, 13(1), e0191870.

Braun, F., Proença, M., Lemay, M., Bertschi, M., Adler, A., Thiran, J.-P., \& Solà, J. (2018b). Limitations and challenges of EIT-based monitoring of stroke volume and pulmonary artery pressure. Physiological Measurement, 39(1), 014003.

Brown, B. H. (2003). Electrical impedance tomography (EIT): A review. Journal of Medical Engineering 83 Technology, 27(3), 97-108.

Brown, B. H., Leathard, A., Sinton, A., McArdle, F. J., Smith, R. W. M., \& Barber, D. C. (1992). Blood flow imaging using electrical impedance tomography. Clinical Physics and Physiological Measurement, 13, 175-179. 
Cheney, M., Isaacson, D., Newell, J. C., Simske, S., \& Goble, J. (1990). NOSER: An algorithm for solving the inverse conductivity problem. International Journal of Imaging Systems and Technology, 2(2), 66-75.

Cohen, J. (1977). Statistical power analysis for the behavioral sciences (Revised edition.). Academic Press.

Coulombe, N., Gagnon, H., Marquis, F., Skrobik, Y., \& Guardo, R. (2005). A parametric model of the relationship between EIT and total lung volume. Physiological Measurement, 26(4), 401-411.

Czaplik, M., Antink, C. H., Rossaint, R., \& Leonhardt, S. (2014). Application of internal electrodes to the oesophageal and tracheal tube in an animal trial: Evaluation of its clinical and technical potentiality in electrical impedance tomography. Journal of Clinical Monitoring and Computing, 28(3), 299-308.

Deibele, J. M., Luepschen, H., \& Leonhardt, S. (2008). Dynamic separation of pulmonary and cardiac changes in electrical impedance tomography. Physiological Measurement, 29(6), S1-S14.

Denaï, M. A., Mahfouf, M., Mohamad-Samuri, S., Panoutsos, G., Brown, B. H., \& Mills, G. H. (2010). Absolute electrical impedance tomography (aEIT) guided ventilation therapy in critical care patients: Simulations and future trends. IEEE Transactions on Information Technology in Biomedicine, 14(3), 641649. 
Dougall, N. J., Bruggink, S., \& Ebmeier, K. P. (2004). Systematic review of the diagnostic accuracy of 99mtc-HMPAO-SPECT in dementia. The American journal of geriatric psychiatry, 12(6), 554-570.

Driessen, R. S., Raijmakers, P. G., Stuijfzand, W. J., \& Knaapen, P. (2017). Myocardial perfusion imaging with PET. The International Journal of Cardiovascular Imaging, 33(7), 1021-1031.

Duggal, A., Ganapathy, A., Ratnapalan, M., \& Adhikari, N. K. (2015). Pharmacological treatments for acute respiratory distress syndrome: Systematic review. Minerva Anestesiologica, 81(5), 567-588.

Ericsson, E., Tesselaar, E., \& Sjöberg, F. (2016). Effect of electrode belt and body positions on regional pulmonary ventilation- and perfusion-related impedance changes measured by electric impedance tomography. PLoS One, 11(6), e0155913.

Essig, M., Shiroishi, M. S., Nguyen, T. B., Saake, M., Provenzale, J. M., Enterline, D., Anzalone, N., Dörfler, A., Rovira, À., Wintermark, M., \& Law, M. (2013). Perfusion MRI: The five most frequently asked technical questions. AJR. American journal of roentgenology, 200(1), 24-34.

Eyüboğlu, B. M., Brown, B. H., Barber, D. C., \& Seager, A. D. (1987). Localisation of cardiac related impedance changes in the thorax. Clinical Physics and Physiological Measurement, 8(4), 167-173. 
Eyüboğlu, B., Brown, B., \& Barber, D. (1989). In vivo imaging of cardiac related impedance changes. IEEE Engineering in Medicine and Biology Magazine, $8(1), 39-45$.

Fagerberg, A., Stenqvist, O., \& Åneman, A. (2009). Electrical impedance tomography applied to assess matching of pulmonary ventilation and perfusion in a porcine experimental model. Critical Care, 13(2), R34.

Faro, S. H., \& Mohamed, F. B. (2006). Functional MRI basic principles and clinical applications (1st ed. 2006.). Springer New York.

Fedorov, A., Beichel, R., Kalpathy-Cramer, J., Finet, J., Fillion-Robin, J.-C., Pujol, S., Bauer, C., Jennings, D., Fennessy, F., Sonka, M., Buatti, J., Aylward, S., Miller, J. V., Pieper, S., \& Kikinis, R. (2012). 3d slicer as an image computing platform for the quantitative imaging network. Magnetic resonance imaging, 30(9), 1323-1341.

Ferrario, D., Grychtol, B., Adler, A., Sola, J., Bohm, S. H., \& Bodenstein, M. (2012). Toward morphological thoracic EIT: Major signal sources correspond to respective organ locations in CT. IEEE Transactions on Biomedical Engineering, 59(11), 3000-3008.

Foster, K. R., \& Lukaski, H. C. (1996). Whole-body impedance-what does it measure? The American Journal of Clinical Nutrition, 64(3), 388S-396S.

Frerichs, I., Hinz, J., Herrmann, P., Weisser, G., Hahn, G., Quintel, M., \& Hellige, G. (2002). Regional lung perfusion as determined by electrical impedance to- 
mography in comparison with electron beam CT imaging. IEEE Transactions on Medical Imaging, 21(6), 646-652.

Frerichs, I., Amato, M. B. P., Kaam, A. H. v., Tingay, D. G., Zhao, Z., Grychtol, B., Bodenstein, M., Gagnon, H., Böhm, S. H., Teschner, E., Stenqvist, O., Mauri, T., Torsani, V., Camporota, L., Schibler, A., Wolf, G. K., Gommers, D., Leonhardt, S., Adler, A., \& Group, T. s. (2017). Chest electrical impedance tomography examination, data analysis, terminology, clinical use and recommendations: Consensus statement of the TRanslational EIT developmeNt stuDy group. Thorax, 72(1), 83-93.

Frerichs, I., \& Becher, T. (2019). Chest electrical impedance tomography measures in neonatology and paediatrics - a survey on clinical usefulness. Physiological Measurement, 40(5), 054001.

Fu, S. X., Lai, K. L., Si, Y. T., Xu, L., Xiao, M. D., \& Gang, A. (1996). Study of the safe threshold of apneic period in children during anesthesia induction. Journal of Clinical Anesthesia, 8(7), 568-574.

Gandev, G., Rao, H., Rashid, S., Kanagarajan, K., \& Gupta, K. (2005). Comparison between perfusion lung scan and ventilation-perfusion lung scan in patients with pulmonary embolism. Chest, 128(4), 406S-406S.

Gaw, R. L. (2010). The effect of red blood cell orientation on the electrical impedance of pulsatile blood with implications for impedance cardiography (Doctoral dissertation). Queensland University of Technology. 
Geddes, L. A., \& Baker, L. E. (1967). The specific resistance of biological material-a compendium of data for the biomedical engineer and physiologist. Medical and biological engineering, 5(3), 271-293.

Geuzaine, C., \& Remacle, J.-F. (2009). Gmsh: A 3-d finite element mesh generator with built-in pre- and post-processing facilities. International Journal for Numerical Methods in Engineering, 79(11), 1309-1331.

Glasser, S. P., Arnett, D. K., McVeigh, G. E., Finkelstein, S. M., Bank, A. J., Morgan, D. J., \& Cohn, J. N. (1997). Vascular compliance and cardiovascular disease: A risk factor or a marker? American Journal of Hypertension, 10(10), 11751189.

Gómez-Laberge, C., \& Adler, A. (2007). Direct calculation of the electrode movement jacobian for 3d EIT. In H. Scharfetter \& R. Merwa (Eds.), 13th international conference on electrical bioimpedance and the 8th conference on electrical impedance tomography (pp. 364-367). Springer.

Gómez-Laberge, C., \& Adler, A. (2008). Direct EIT jacobian calculations for conductivity change and electrode movement. Physiological Measurement, 29(6), S89-S99.

Gordon, J. W. (1877). Certain molar movements of the human body produced by the circulation of the blood. Journal of Anatomy and Physiology, 11, 533-536.

Graham, B. M., \& Adler, A. (2007). Electrode placement configurations for 3d EIT. Physiological Measurement, 28(7), S29-S44. 
Grychtol, B., \& Adler, A. (2013). FEM electrode refinement for electrical impedance tomography. 2013 35th Annual International Conference of the IEEE Engineering in Medicine and Biology Society (EMBC), 6429-6432.

Grychtol, B., Lionheart, W. R. B., Bodenstein, M., Wolf, G. K., \& Adler, A. (2012). Impact of model shape mismatch on reconstruction quality in electrical impedance tomography. IEEE Transactions on Medical Imaging, 31(9), $1754-1760$.

Grychtol, B., Müller, B., \& Adler, A. (2016). 3d EIT image reconstruction with GREIT. Physiological Measurement, 37(6), 785-800.

Halter, R., Hartov, A., \& Paulsen, K. (2008). Imaging forearm blood flow with pulseox gated electrical impedance tomography. 2008 30th Annual International Conference of the IEEE Engineering in Medicine and Biology Society, 11921195.

Hochhausen, N., Orschulik, J., Follmann, A., Santos, S. A., Dohmeier, H., Leonhardt, S., Rossaint, R., \& Czaplik, M. (2019). Comparison of two experimental ARDS models in pigs using electrical impedance tomography. PLoS ONE, 14(11), $\mathrm{e} 0225218$.

Holder, D. S. (1992). Electrical impedance tomography (EIT) of brain function. Brain Topography, 5(2), 87-93.

Holder, D. (Ed.). (2004). Electrical impedance tomography: Methods, history and applications. CRC Press. 
Hollwarth, M. E., Smith, M., Kvietys, P. R., \& Granger, D. N. (1986). Esophageal blood flow in the cat. Gastroenterology, 90(3), 622-627.

Hough, J., Trojman, A., \& Schibler, A. (2016). Effect of time and body position on ventilation in premature infants. Pediatric Research, 80(4), 499-504.

Hsu, Y.-L., Tien, A.-J., Chang, M.-Y., Chang, H.-T., Möller, K., Frerichs, I., \& Zhao, Z. (2017). Regional ventilation redistribution measured by electrical impedance tomography during spontaneous breathing trial with automatic tube compensation. Physiological Measurement, 38(6), 1193-1203.

Humphreys, S., Pham, T. M., Stocker, C., \& Schibler, A. (2011). The effect of induction of anesthesia and intubation on end-expiratory lung level and regional ventilation distribution in cardiac children. Pediatric Anesthesia, 21(8), 887893.

Hur, S., Bauer, A., McMillan, N., Krupinski, E. A., \& Kuo, P. H. (2014). Optimizing the ventilation-perfusion lung scan for image quality and radiation exposure. Journal of Nuclear Medicine Technology, 42(1), 51-54.

International Electrotechnical Commission, 2. (2021). IEC 60601-1. International Electrotechnical Commission.

Jaccard, P. (1912). The distribution of the flora in the alpine zone.1. New Phytologist, 11(2), 37-50.

Jackson, A., Buckley, D. L., \& Parker, G. J. M. (2005). Dynamic contrast-enhanced magnetic resonance imaging in oncology (1st ed. 2005.). Springer Berlin Heidelberg. 
Kim, C.-S., Ober, S. L., McMurtry, M. S., Finegan, B. A., Inan, O. T., Mukkamala, R., \& Hahn, J.-O. (2016). Ballistocardiogram: Mechanism and potential for unobtrusive cardiovascular health monitoring. Scientific Reports, 6, 31297.

Kircher, M., Elke, G., Stender, B., Hernández Mesa, M., Schuderer, F., Dössel, O., Fuld, M. K., Halaweish, A. F., Hoffman, E. A., Weiler, N., \& Frerichs, I. (2021). Regional lung perfusion analysis in experimental ARDS by electrical impedance and computed tomography. IEEE Transactions on Medical Imaging, 40(1), 251-261.

Koenig, M., Klotz, E., Luka, B., Venderink, D. J., Spittler, J. F., \& Heuser, L. (1998). Perfusion CT of the brain: Diagnostic approach for early detection of ischemic stroke. Radiology, 209(1), 85-93.

Kolehmainen, V., Vauhkonen, M., Karjalainen, P. A., \& Kaipio, J. P. (1997). Assessment of errors in static electrical impedance tomography with adjacent and trigonometric current patterns. Physiological Measurement, 18(4), 289-303.

Konstas, A. A., Goldmakher, G. V., Lee, T.-Y., \& Lev, M. H. (2009). Theoretic basis and technical implementations of CT perfusion in acute ischemic stroke, part 1: Theoretic basis. American Journal of Neuroradiology, 30(4), 662-668.

Koretsky, A. P. (2012). Early development of arterial spin labeling to measure regional brain blood flow by MRI. NeuroImage, 62(2), 602-607.

Larsson, J. (2007). Electromagnetics from a quasistatic perspective. American Journal of Physics, 75(3), 230-239. 
Leathard, A. D., Brown, B. H., Campbell, J., Zhang, F., Morice, A. H., \& Tayler, D. (1994). A comparison of ventilatory and cardiac related changes in EIT images of normal human lungs and of lungs with pulmonary emboli. Physiological Measurement, 15(2), A137-A146.

Leonhardt, S., \& Lachmann, B. (2012). Electrical impedance tomography: The holy grail of ventilation and perfusion monitoring? Intensive Care Medicine, 38(12), 1917-1929.

Lozano, A., Rosell, J., \& Pallas-Areny, R. (1995). Errors in prolonged electrical impedance measurements due to electrode repositioning and postural changes. Physiological Measurement, 16(2), 121-130.

Malone, E., dos Santos, G. S., Holder, D., \& Arridge, S. (2014). Multifrequency electrical impedance tomography using spectral constraints. IEEE Transactions on Medical Imaging, 33(2), 340-350.

McAdams, E. T., \& Jossinet, J. (1995). Tissue impedance: A historical overview. Physiological Measurement, 16(3), A1-A13.

Mendis, S., Puska, P., \& Norrving, B. (2011). Global atlas on cardiovascular disease prevention and control. World Health Organization.

Mettler, F. A., \& Guiberteau, M. J. (2006). Essentials of nuclear medicine imaging (5th ed.). Saunders/Elsevier.

Miles, K. A., Hayball, M., Dixon, A. K., Miles, K. A., Hayball, M., \& Dixon, A. K. (1991). Colour perfusion imaging: A new application of computed tomography. The Lancet, 337(8742), 643-645. 
Moens, Y., Schramel, J. P., Tusman, G., Ambrisko, T. D., Solà, J., Brunner, J. X., Kowalczyk, L., \& Böhm, S. H. (2014). Variety of non-invasive continuous monitoring methodologies including electrical impedance tomography provides novel insights into the physiology of lung collapse and recruitment case report of an anaesthetized horse. Veterinary Anaesthesia and Analgesia, 41(2), 196-204.

Mortensen, J., \& Berg, R. M. G. (2019). Lung scintigraphy in COPD. Seminars in Nuclear Medicine, 49(1), 16-21.

Nakazato, R., Tamarappoo, B. K., Kang, X., Wolak, A., Kite, F., Hayes, S. W., Thomson, L. E. J., Friedman, J. D., Berman, D. S., \& Slomka, P. J. (2010). Quantitative upright-supine high-speed SPECT myocardial perfusion imaging for detection of coronary artery disease: Correlation with invasive coronary angiography. The Journal of Nuclear Medicine, 51(11), 1724-31.

Nasehi Tehrani, J., Jin, C., \& McEwan, A. L. (2012a). Modelling of an oesophageal electrode for cardiac function tomography. Computational and Mathematical Methods in Medicine, 2012, e585786.

Nasehi Tehrani, J., Oh, T. I., Jin, C., Thiagalingam, A., \& McEwan, A. (2012b). Evaluation of different stimulation and measurement patterns based on internal electrode: Application in cardiac impedance tomography. Computers in Biology and Medicine, 42(11), 1122.

Nguyen, D. T., Bhaskaran, A., Chik, W., Barry, M. A., Pouliopoulos, J., Kosobrodov, R., Jin, C., Oh, T. I., Thiagalingam, A., \& McEwan, A. L. (2015). Perfusion 
redistribution after a pulmonary-embolism-like event with contrast enhanced EIT. Physiological Measurement, 36(6), 1297-1309.

Nguyen, D. T., Jin, C., Thiagalingam, A., \& McEwan, A. L. (2012). A review on electrical impedance tomography for pulmonary perfusion imaging. Physiological Measurement, 33(5), 695-706.

Nguyen, D. M., Andersen, T., Qian, P., Barry, T., \& McEwan, A. (2020). Electrical impedance tomography for monitoring cardiac radiofrequency ablation: A scoping review of an emerging technology. Medical Engineering 85 Physics, 84, $36-50$.

Nissinen, A., Heikkinen, L. M., Kolehmainen, V., \& Kaipio, J. P. (2009). Compensation of errors due to discretization, domain truncation and unknown contact impedances in electrical impedance tomography. Measurement Science and Technology, 20(10), 105504.

Nyboer, J., Bagno, S., Barnett, A., \& Halsey, R. H. (1970). Impedance cardiograms and differentiated-impedance cardiograms-the electrical impedance changes of the heart in relation to electrocardiograms and heart sounds*. Annals of the New York Academy of Sciences, 170(2), 421-436.

Ostebee, A. (1998). Rank-deficient and discrete ill-posed problems: Numerical aspects of linear inversion. The American Mathematical Monthly, 105(5), 491.

Pappano, A. J., \& Wier, W. G. (2019). Cardiovascular physiology (11th ed.). Elsevier.

Patterson, R. P. (2010). Impedance cardiography: What is the source of the signal? Journal of Physics: Conference Series, 224, 012118. 
Patterson, R. P., Zhang, J., Mason, L. I., \& Jerosch-Herold, M. (2001). Variability in the cardiac EIT image as a function of electrode position, lung volume and body position. Physiological Measurement, 22(1), 159-166.

Petty, T. L. (2001). In the cards was ARDS. American Journal of Respiratory and Critical Care Medicine, 163(3), 602-603.

Pham, T., \& Rubenfeld, G. D. (2017). Fifty years of research in ARDS. the epidemiology of acute respiratory distress syndrome. a 50th birthday review. American Journal of Respiratory and Critical Care Medicine, 195(7), 860-870.

Pilkington, T., Eyüboğlu, M., \& Wolf, P. (1989). Utilization of esophageal reference electrode to enhance impedance imaging. Images of the Twenty-First Century. Proceedings of the Annual International Engineering in Medicine and Biology Society, 482 vol.2--.

PIOPED-Investigators. (1990). Value of the ventilation/perfusion scan in acute pulmonary embolism: Results of the prospective investigation of pulmonary embolism diagnosis (PIOPED). JAMA, 263(20), 2753-2759.

Pollock, J. D., \& Makaryus, A. N. (2021). Physiology, cardiac cycle. Physiology, cardiac cycle. StatPearls Publishing.

Polydorides, N., \& McCann, H. (2002). Electrode configurations for improved spatial resolution in electrical impedance tomography. Measurement Science and Technology, 13(12), 1862-1870.

Proença, M., Braun, F., Lemay, M., Solà, J., Adler, A., Riedel, T., Messerli, F. H., Thiran, J.-P., Rimoldi, S. F., \& Rexhaj, E. (2020). Non-invasive pulmonary 
artery pressure estimation by electrical impedance tomography in a controlled hypoxemia study in healthy subjects. Scientific Reports, 10(1), 21462.

Proença, M., Braun, F., Rapin, M., Solà, J., Adler, A., Grychtol, B., Bohm, S. H., Lemay, M., \& Thiran, J.-P. (2015). Influence of heart motion on cardiac output estimation by means of electrical impedance tomography: A case study. Physiological Measurement, 36(6), 1075-1091.

Proença, M., Braun, F., Solà, J., Thiran, J.-p., \& Lemay, M. (2017). Noninvasive pulmonary artery pressure monitoring by EIT: A model-based feasibility study. Medical and Biological Engineering and Computing, 55(6), 949-963.

Prvulovich, E. M., \& Bomanji, J. B. (1998). The role of nuclear medicine in clinical investigation. BMJ : British Medical Journal, 316(7138), 1140-1146.

Reifferscheid, F., Elke, G., Pulletz, S., Gawelczyk, B., Lautenschläger, I., Steinfath, M., Weiler, N., \& Frerichs, I. (2011). Regional ventilation distribution determined by electrical impedance tomography: Reproducibility and effects of posture and chest plane. Respirology, 16(3), 523-531.

Sage, M., Nadeau, M., Forand-Choinière, C., Mousseau, J., Vandamme, J., Berger, C., Tremblay-Roy, J.-S., Tissier, R., Micheau, P., \& Fortin-Pellerin, É. (2018). Assessing the impacts of total liquid ventilation on left ventricular diastolic function in a model of neonatal respiratory distress syndrome. PLoS One, 13(1), e0191885.

Sanchez, B., Vandersteen, G., Martin, I., Castillo, D., Torrego, A., Riu, P. J., Schoukens, J., \& Bragos, R. (2013). In vivo electrical bioimpedance character- 
ization of human lung tissue during the bronchoscopy procedure. a feasibility study. Medical Engineering \& Physics, 35(7), 949-957.

Schöberl, J. (1997). NETGEN an advancing front 2d/3d-mesh generator based on abstract rules. Computing and Visualization in Science, 1(1), 41-52.

Schuessler, T., \& Bates, J. (1995). Utility of an esophageal reference electrode for thoracic electrical impedance tomography. Proceedings of 17 th International Conference of the Engineering in Medicine and Biology Society, 1, 559-560 vol.1.

Smit, H. J., Handoko, M. L., Noordegraaf, A. V., Faes, T. J. C., Postmus, P. E., Vries, P. M. J. M. d., \& Boonstra, A. (2003). Electrical impedance tomography to measure pulmonary perfusion: Is the reproducibility high enough for clinical practice? Physiological Measurement, 24(2), 491-499.

Solà, J., Adler, A., Santos, A., Tusman, G., Sipmann, F. S., \& Bohm, S. H. (2011). Non-invasive monitoring of central blood pressure by electrical impedance tomography: First experimental evidence. Medical and Biological Engineering and Computing, 49(4), 409-15.

Soleimani, M., Gómez-Laberge, C., \& Adler, A. (2006). Imaging of conductivity changes and electrode movement in EIT. Physiological Measurement, 27(5), S103-S113.

Somersalo, E., Cheney, M., \& Isaacson, D. (1992). Existence and uniqueness for electrode models for electric current computed tomography. SIAM Journal on Applied Mathematics, 52(4), 18. 
Sourbron, S. P., \& Buckley, D. L. (2013). Classic models for dynamic contrast-enhanced MRI. NMR in Biomedicine, 26(8), 1004-1027.

Šribar, A., Merc, V., Peršec, Z., Peršec, J., Milas, I., \& Husedžinović, S. (2020). Influence of different PEEP levels on electrical impedance tomography findings in patients under general anesthesia ventilated in the lateral decubitus position. Journal of Clinical Monitoring and Computing, 34(2), 311-318.

Stowe, S., \& Adler, A. (2020). The effect of internal electrodes on electrical impedance tomography sensitivity. 2020 42nd Annual International Conference of the IEEE Engineering in Medicine Biology Society (EMBC), 1457-1460.

Stowe, S., Boyle, A., Sage, M., See, W., Praud, J.-P., Fortin-Pellerin, É., \& Adler, A. (2019). Comparison of bolus- and filtering-based EIT measures of lung perfusion in an animal model. Physiological Measurement, 40(5), 054002.

Stowe, S., Samson, N., Nadeau, C., Morin, C., Praud, J.-P., Fortin-Pellerin, É., \& Adler, A. (2021a). Using esophageal electrodes for increased sensitivity to cardiac-frequency impedance changes. Proceedings of the 21st International Conference on Biomedical Applications of Electrical Impedance Tomography, 24.

Stowe, S., Zhao, Z., Huxter, E., He, H., Long, Y., \& Adler, A. (2021b). Generating forward models from CT images for ventilation monitoring in ARDS patients. Proceedings of the 21st International Conference on Biomedical Applications of Electrical Impedance Tomography, 20. 
Vauhkonen, M., Vadasz, D., Karjalainen, P., Somersalo, E., \& Kaipio, J. (1998). Tikhonov regularization and prior information in electrical impedance tomography. IEEE Transactions on Medical Imaging, 17(2), 285-293.

Vauhkonen, P. J., Vauhkonen, M., Savolainen, T., \& Kaipio, J. P. (1999). Threedimensional electrical impedance tomography based on the complete electrode model. IEEE transactions on bio-medical engineering, 46(9), 1150-1160.

Vijayalakshmi, K., Kelly, D., Chapple, C.-L., Williams, D., Wright, R., Stewart, M. J., Hall, J. A., Sutton, A., Davies, A., Haywood, J., \& de Belder, M. A. (2007). Cardiac catheterisation: Radiation doses and lifetime risk of malignancy. Heart, 93(3), 370-371.

Vonk Noordegraaf, A., Faes, T. J. C., Janse, A., Marcus, J. T., Heethaar, R. M., Postmus, P. E., \& Vries, P. M. J. M. d. (1996). Improvement of cardiac imaging in electrical impedance tomography by means of a new electrode configuration. Physiological Measurement, 17(3), 179-188.

Vonk Noordegraaf, A., Kunst, P. W. A., Janse, A., Marcus, J. T., Postmus, P. E., Faes, T. J. C., \& Vries, P. M. J. M. d. (1998). Pulmonary perfusion measured by means of electrical impedance tomography. Physiological Measurement, $19(2), 263-273$.

Waldmann, A. D., Wodack, K. H., März, A., Ukere, A., Trepte, C. J., Böhm, S. H., \& Reuter, D. A. (2017). Performance of novel patient interface for electrical impedance tomography applications. Journal of Medical and Biological Engineering, 37(4), 561-566. 
Watson, R. E. (2015). Lessons learned from MRI safety events. Current Radiology Reports, 3(10), 37.

Witsoe, D. A., \& Kinnen, E. (1967). Electrical resistivity of lung at $100 \mathrm{kHz}$. Medical and biological engineering, 5(3), 239-248.

Yang, L., Dai, M., Möller, K., Frerichs, I., Adler, A., Fu, F., \& Zhao, Z. (2021). Lung regions identified with CT improve the value of global inhomogeneity index measured with electrical impedance tomography. Quantitative Imaging in Medicine and Surgery, 11(4), 1209-1219.

Zadehkoochak, M., Blott, B. H., Hames, T. K., \& George, R. F. (1992). Pulmonary perfusion and ventricular ejection imaging by frequency domain filtering of EIT images. Clinical Physics and Physiological Measurement, 13, 191-196.

Zhao, Z., Fischer, R., Frerichs, I., Müller-Lisse, U., \& Möller, K. (2012). Regional ventilation in cystic fibrosis measured by electrical impedance tomography. Journal of Cystic Fibrosis, 11(5), 412-418.

Zhao, Z., Möller, K., Steinmann, D., Frerichs, I., \& Guttmann, J. (2009). Evaluation of an electrical impedance tomography-based global inhomogeneity index for pulmonary ventilation distribution. Intensive Care Medicine, 35(11). 
Appendices 


\section{Appendix A}

\section{GMSH models}

In order to create models with mesh refinement in GMSH matlab was used to generate geometry (or .geo) files. Examples of the geometry files for a basic tank with 4 square external electrodes and the internal probe with muptiple densitities are presented. To use these models in EIDORS they must be meshed using GMSH and loaded into eidors with gmsh_read_mesh. Also see mat_idx_2_electrode to specify electrodes in GMSH meshes. To run these meshes with gmsh from the command line copy the text into a file and type: gmsh <filename.geo $>$ while in the same directory.

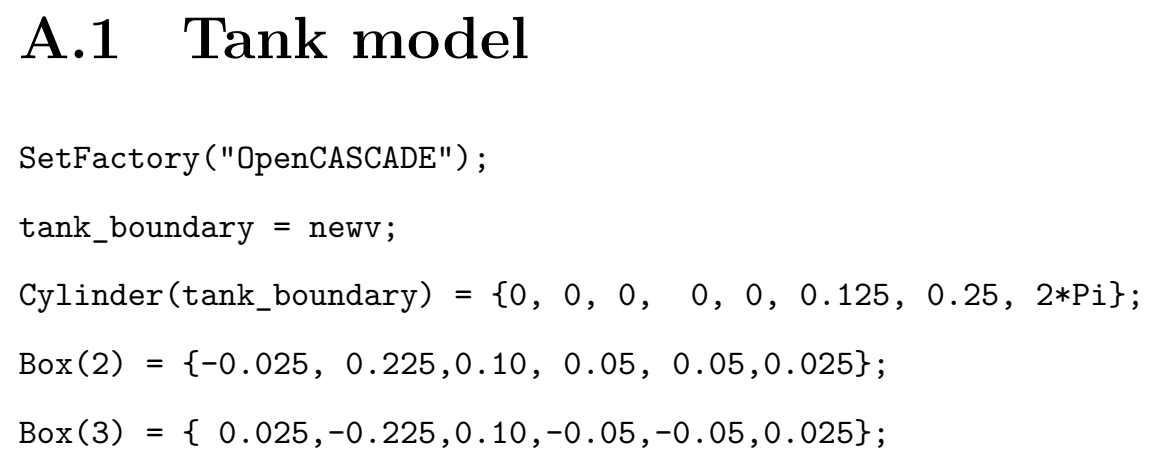




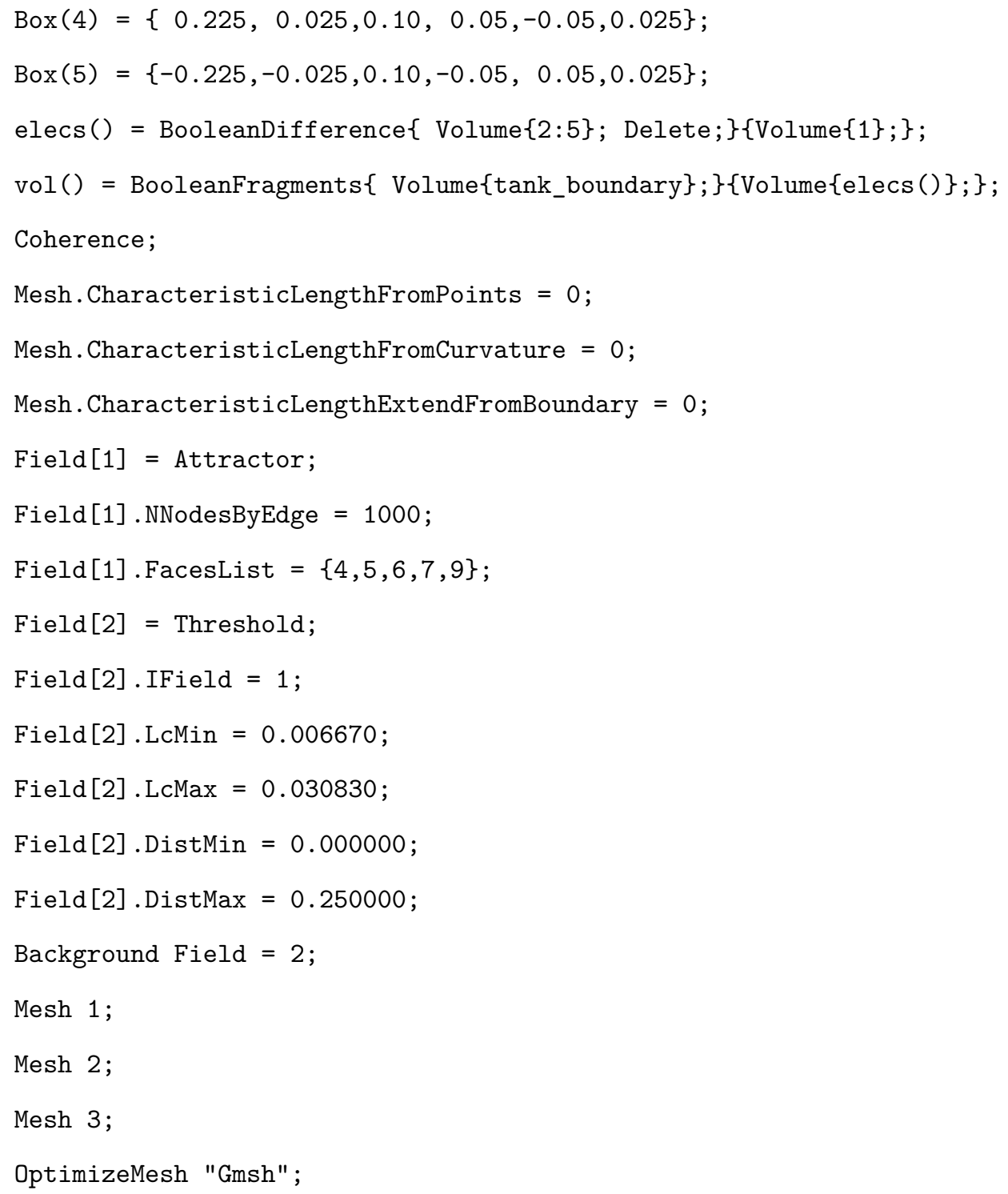

\section{A.2 Internal probe model}

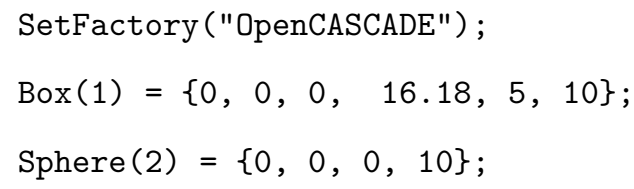




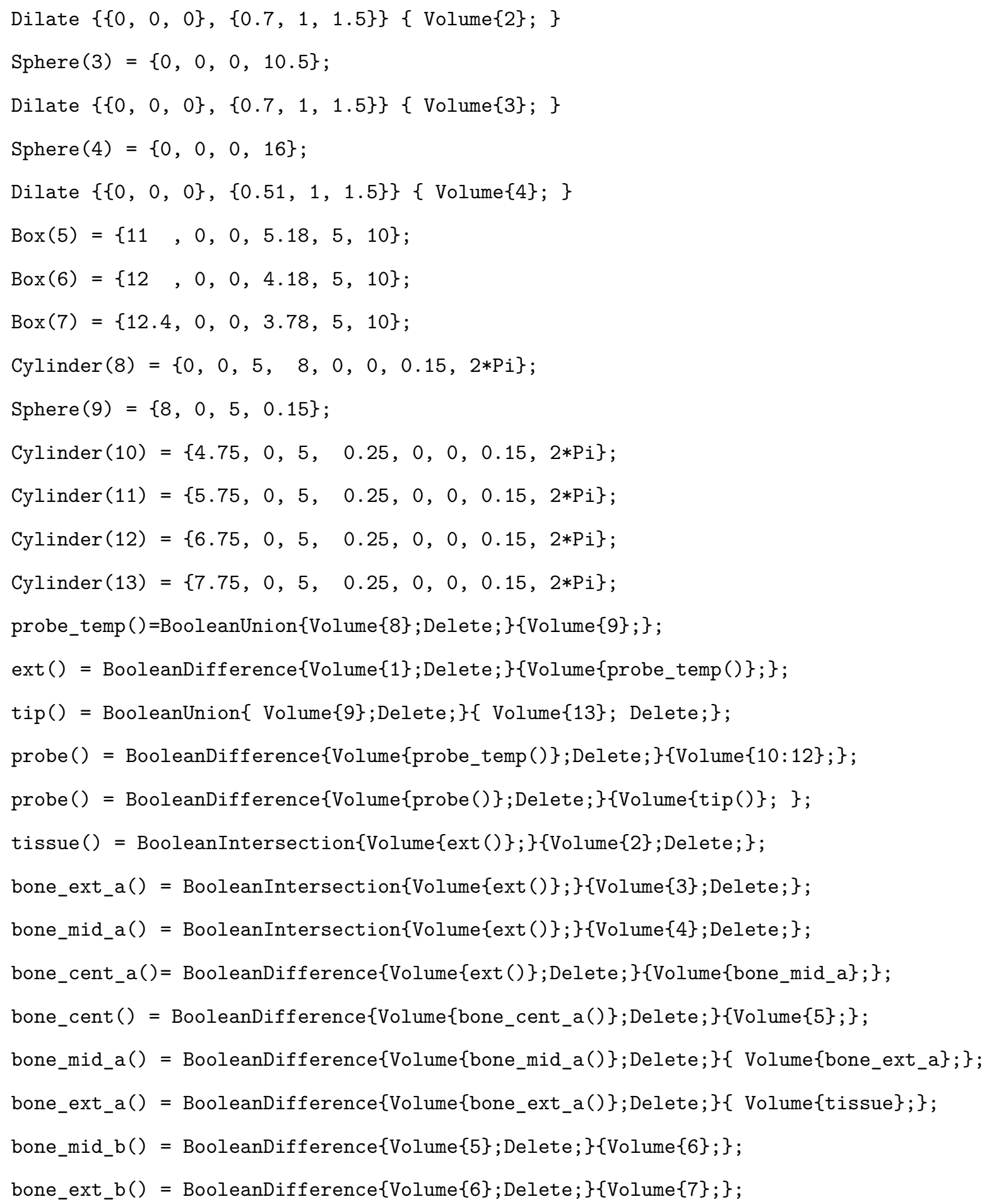




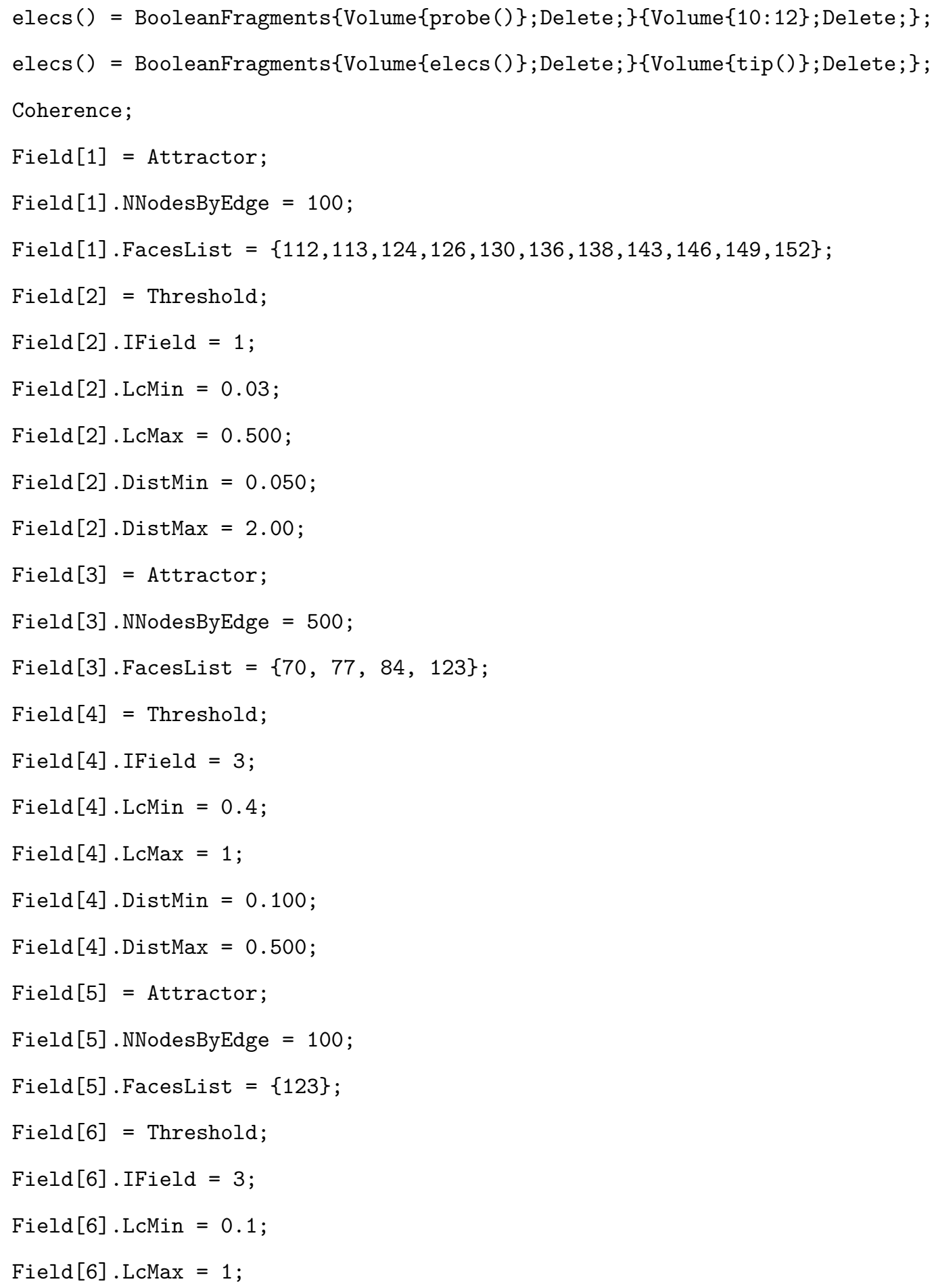


Field [6] $\cdot$ DistMin $=0.100 ;$

Field[6].DistMax $=0.500$;

Field [7] = Min;

Field[7].FieldsList $=\{2,4,6\}$;

Background Field = 7;

Mesh 1;

Mesh 2;

Mesh 3;

Save 'advanced_mesh.msh'; 


\section{Appendix B}

\section{Algorithms}

The following sections show the segmentation methods from chapter 5 presented as algorithms for a clear presentation of the segmentation steps.

\section{B.1 External boundary}

The following steps were used to segment the external boundary from a single slice of a CT image using Matlab 2021b with the image processing toolbox: 


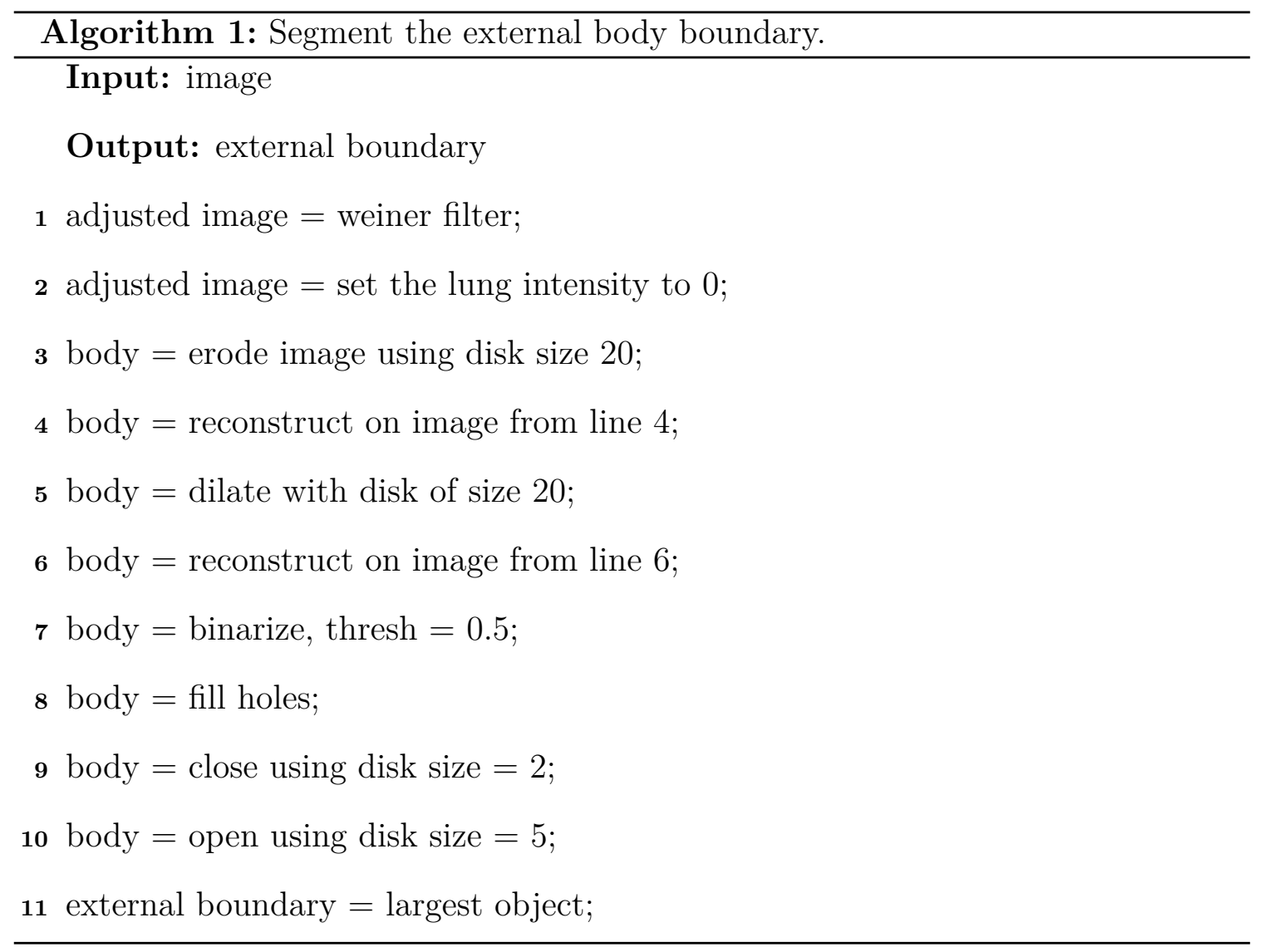

\section{B.2 Ribcage}

The below algorithm is a presnetation of the segmentation methods to extract the ribcage from a selected CT slice. 


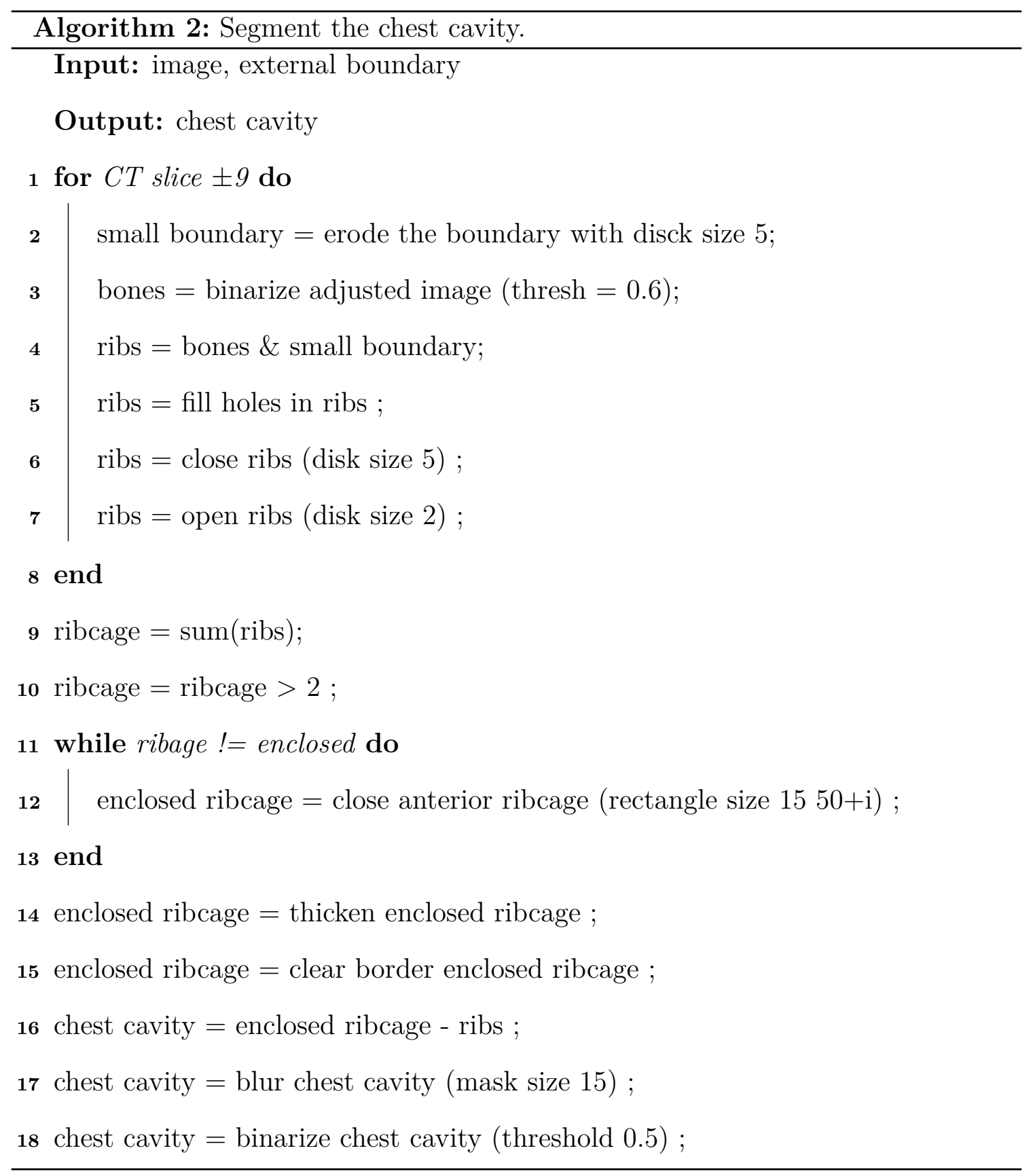




\section{B.3 Lungs}

This algorithm shoes the final steps taken to segment the lungs using the chest cavity segmentation.

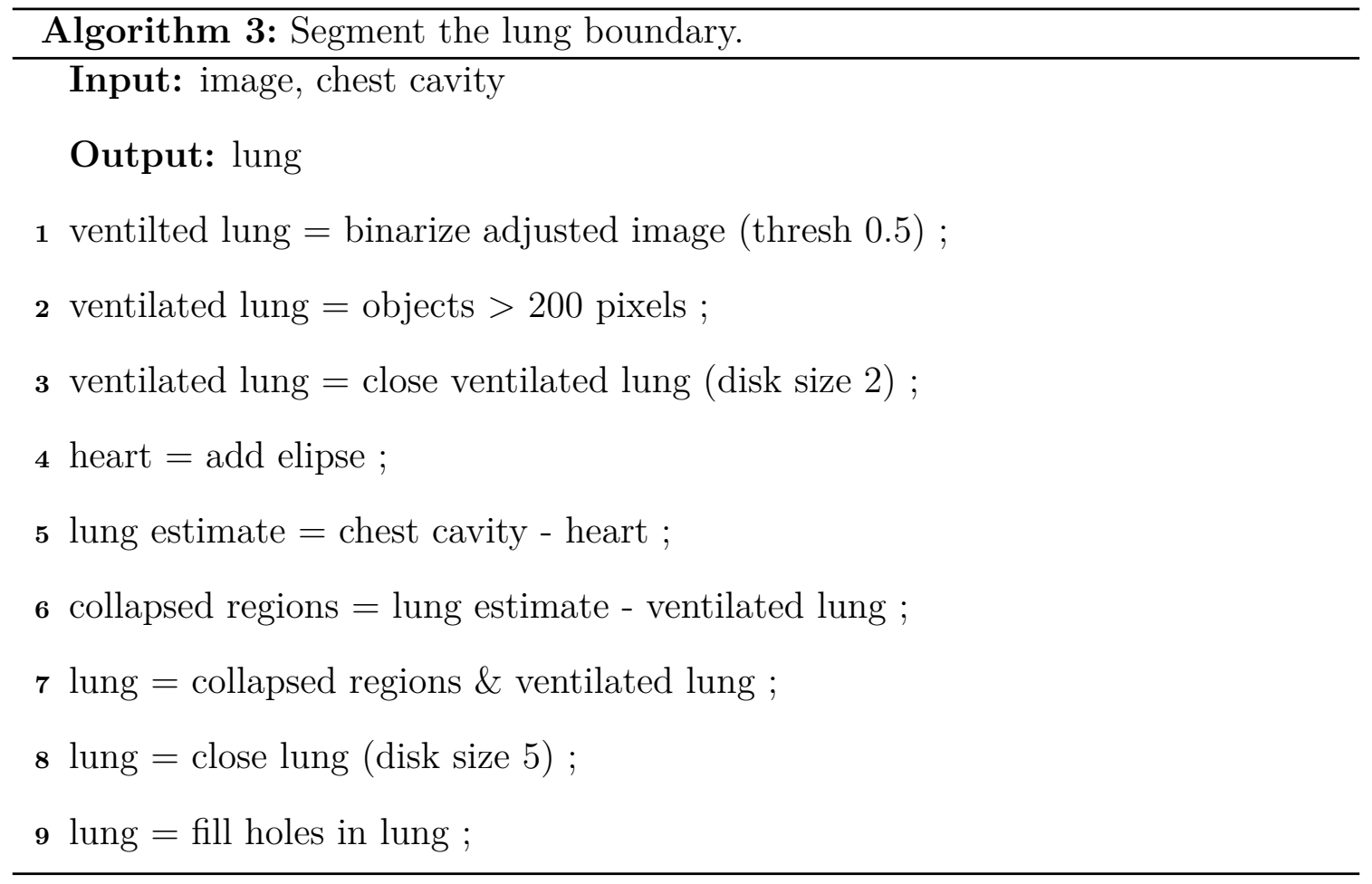

